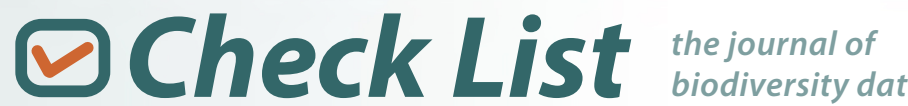

\section{Inventory of butterfly fauna (Lepidoptera: Rhopalocera) of Tripura, India, in the Indo-Myanmar biogeographical zone, with records of threatened taxa}

\section{Rahul Lodh and Basant Kumar Agarwala*}

\begin{abstract}
Tripura University, Department of Zoology, Ecology and Biodiversity Laboratories, Suryamaninagar, Tripura, India, 799022
* Corresponding author: E-mail: bagarwala00@gmail.com
\end{abstract}

\begin{abstract}
The Tripura province of northeast India, situated in the western fringe of the Indo-Myanmar biodiversity hotspot was extensively explored for butterfly fauna. We surveyed 28 wild and managed locations in the hot and humid environment of Tripura province (2009-2012) across different landscape gradients to observe and record butterfly diversity. We report here 212 butterfly species, 65 of which are considered threatened including 41 species protected by Indian Wildlife Protection Act 1972. Thirty-eight species are categorized as threatened by IUCN including one species also by CITES. We recorded the abundance of each butterfly species to highlight the population status of individual butterfly species in order to reveal the status of their conservation in Tripura, in India, and also at global scale. Geographic distribution of 142 species is extended to Tripura. Results of this study form a baseline data on the diversity and conservation of butterflies in this biodiversity hotspot.
\end{abstract}

Key words: butterfly, conservation, northeast India, threatened species, Tripura

\section{INTRODUCTION}

Insects constitute more than half of the world's known biodiversity (May 1992), perform multidirectional activities, viz., pollination, energy transformation, seed dispersal, and contribute to the productivity and stability of ecosystem (Naeem et al. 1994; Tilman et al. 1996). This is particularly true for tropical regions where insects show high abundance and species diversity (Samways 2005; Spector 2006). Among insects, butterflies are a taxonomically well studied group throughout the world (Ghazoul 2002) and are indicator taxa in terms of habitat quality and anthropogenic disturbance (Kocher and Williams 2000). More than 18,000 butterfly species have been documented worldwide (Heppner 1998; Larsen et al. 2011; Martinez et al. 2003) including 1501 species reported from India (Kehimkar 2008). Northeastern parts of India, consisting of eight provinces and having an area of $1,61,419 \mathrm{~km}^{2}$, shares about $65 \%$ of butterfly diversity of India (Haribal 1992; Gupta and Majumder 2006; Gupta and Maulik 2007; Gogoi 2012; Kunte et al. 2012). However, in geographical scale and in terms of species richness, the Tripura province (area: 10,491 km²) had 72 species known (Agarwala et al. 2010; Majumder J. et al. 2012b; Roy Choudhury et al. 2011). This is far less in comparison to Sikkim, a smaller province having an area of 7,092 $\mathrm{km}^{2}$ but with 695 species of butterflies known (Haribal 1992).

Tripura province of northeastern India is situated in the western fringe of Indo-Myanmar global hotspot (Mittermeier et al. 2005; Tordoff et al. 2012). It is rich in forests (ca. $57.73 \%$ of the total land area) and is dominated by semi-evergreen to moist deciduous plants and secondary bamboo brakes (Majumdar K. et al. 2012a). The richness of biodiversity of the province is evident from occurrence of 1,583 plant species belonging to 862 genera in 193 families (Deb 1981-1983; Majumdar K. et al. 2012b), 90 species of mammals (Gupta 1998), 17 species of amphibians, 35 species of reptiles (Majumder J. et al. 2012a) and 300 species of birds (Choudhury 2010; Bhattacharjee et al. 2013). Tripura province is unique due its location at the confluence of Indo-Myanmar, IndoMalayan and parts of Indo-China geographical regions with close proximity to Bangladesh. The presence of mighty Brahmaputra River, originating in China and flows through Assam in the north and east of Tripura, acts as a barrier for gene flow from south-east Asian provinces, including Tripura (Datta-Roy et al. 2012).

To bridge the gap of information on the butterfly fauna, a sustained exploration was undertaken from June 2009 to December 2012 across different habitat gradients of the province. 


\section{MATERIAL AND METHODS}

\section{Study area}

In Southeast Asia, Tripura province of India lies between $22^{\circ} 56^{\prime}$ to $24^{\circ} 32^{\prime} \mathrm{N}$ and $091^{\circ} 09^{\prime}$ to $092^{\circ} 20^{\prime} \mathrm{E}$, and represents the western fringe of Indo-Myanmar biogeographic zone of tropical Asia (Myers et al. 2000; Mittermeier et al. 2005). The province has an area of $10,490 \mathrm{~km}^{2}$, is largely forested along with its adjacent provinces of Mizoram, Manipur, Meghalaya and Assam in India, forms part of biodiversity hotspot, and is predominantly hilly with five hills ranges (elevations between 244-939 $\mathrm{m}$ above mean sea level) which run parallel from north to south. These ranges from west to east are: the Boromura-Deotamura, Atharamura, Longtharai, Shakhan, and Jampui hills. They decrease in elevation southwards and finally merge into the eastern plains. Four major valleys intersect these hill ranges: Dharmanagar-Kanchanpur, Kailasahar-Manu, Kamalpur-Ambasa and Khowai-Teliamura valleys (Figure 1). Tripura has secondary moist mixed deciduous forest in northern parts, and widely distributed moist deciduous teak forest in the south district and part of west district (Majumdar K. et al. 2012b). Four main seasons

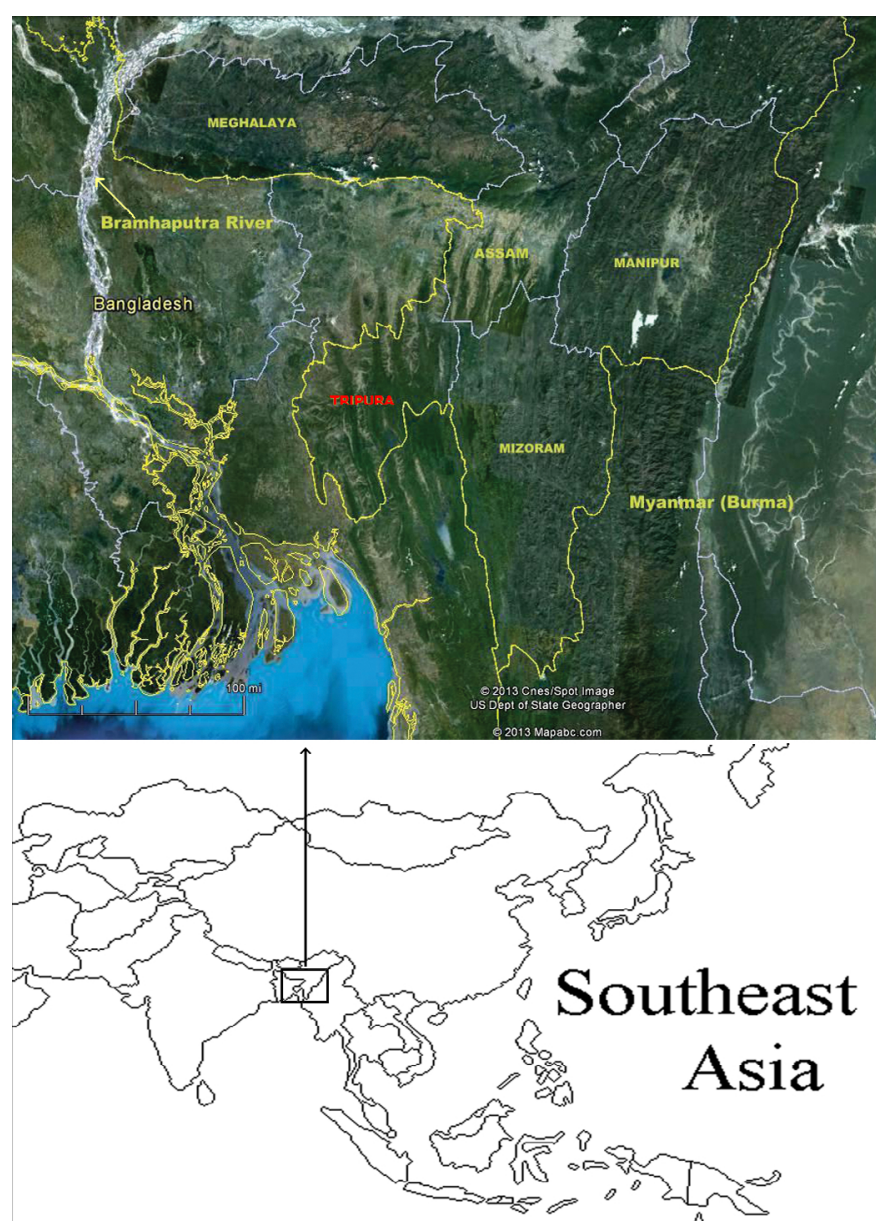

Figure 1. Line drawning of Southeast Asia and google earth image, captured on 3 May 2013, showing location of the study area, Tripura province and its adjoining provinces (Mizoram, Meghalaya, Assam) in India and adjacent countries (Bangladesh, Myanmar), in the western fringe of IndoMyanmar hotspot region. are observed: winter (late November to February), summer (March to May), monsoon (June to September) and autumn (October to mid November). Average annual temperature varies from the minimum average of $10^{\circ} \mathrm{C}$ in winter to the maximum average of $35^{\circ} \mathrm{C}$ in summer. During monsoon season, south-west monsoons bring maximum rainfall $(65 \%)$ to the province. The average annual rainfall is 2,000-2,500 $\mathrm{mm}$. The average humidity during the summer is $50-74 \%$; during the monsoon season, it is above $85 \%$ (Keshari 2011).

The butterfly fauna at 28 locations in wild and managed habitats were inventoried. These were distributed in all the eight administrative districts of Tripura (Figure 2). Geo-coordinates and forest types are provided in Table 1 for each location (with their abbreviated names used in the systematic list of species recorded in this study). Among the 28 locations, 12 are located in South district and Gomati district, which have the maximum forest cover in the province $\left(2,473 \mathrm{~km}^{2}\right)$ as well as three protected reserves, Trishna Wildlife Sanctuary (163.08 $\left.\mathrm{km}^{2}\right)$, Rajbari National Park $\left(31.63 \mathrm{~km}^{2}\right.$ ) and Gomati Wildlife Sanctuary $\left(389.54 \mathrm{~km}^{2}\right)$. Another four locations are in Sepahijala district which includes Sepahijala Wildlife Sanctuary $\left(18.53 \mathrm{~km}^{2}\right)$ and Clouded Leopard National Park. Four locations are in the North district that includes one protected reserve (Rowa Wildlife Sanctuary; $0.85 \mathrm{~km}^{2}$ ). Among rest of the locations, three locations are distributed in West district, two locations in Khowai district, two locations in Dhalai district, and one location in Unakoti district (Figure 2).

\section{Sampling Method}

Two transect lines, each $1,000 \mathrm{~m}$ long $\times 10 \mathrm{~m}$ wide, using permanent markers (nylon rope tied to tree trunks at regular intervals along the transect) were laid at each of the 28 locations. These transects were visited twice in a year (once each in summer and winter) during the study period of three years: 2009-2010, 2010-2011, and 2011-2012. Transect lines were laid at sites that were rich in vegetation and showed a matrix of microhabitats. Transects were shifted every year within each location to give wider effect of exploration. As a result, six transects (two transects/year for three years) were laid at each location and these were visited 12 times during the study period. Thus, a total of 168 transects ( 28 locations $\times 6$ transects) measuring $40.99 \mathrm{~km}^{2}$ area (168 transects $\times 1000 \mathrm{~m}$ length $\times 10 \mathrm{~m}$ width) was covered for sampling following the Modified Pollard Walk Method (MPWM) (Pollard 1977). To maximize the encounter rate of butterflies, 10 minutes stops were made at every 100 $\mathrm{m}$ interval along transects and butterflies were observed within $10 \mathrm{~m}$ radius in all sides. Observations were done on sunny days between o6:00 $\mathrm{h}$ and 12:00 $\mathrm{h}$ local time on two consecutive days.

For each species, identifications were made and 
numbers of individuals were counted. Photographs were taken when convenient, using high zoom Canon 50D and Lumix DMC FZ4o cameras. Butterfly species that could not be identified on flight were captured using butterfly net and released immediately after identification of sex and species, using field guides (Haribal 1992; Kunte 2000; Kehimkar 2008).

At least one individual of every available species that were encountered were collected for taxonomic study with prior permission by the Department of Forests, Government of Tripura. All the specimens of this study were spread and preserved following the standard taxonomic procedure (Winston 1999). Butterflies are classified according to Ackery (1984), Campbell et al. (2000) and Savela (2013). Monomorphic butterfly species that were collected or photographed were not identified by sex; therefore, they are tagged as male or female $(\hat{\partial} /+)$. Sexually polymorphic species that were collected or photographed are tagged either as male $\left({ }^{\top}\right)$ or female (ㅇ). All specimens and photographs used in the study were given voucher numbers and are maintained in the Ecology and Biodiversity Laboratory, Department of Zoology, Tripura University, India.

Based on accumulated data of the three years of study, abundance of each of the recorded butterfly species is considered into five categories: very common represented by $>200$ individuals, common represented

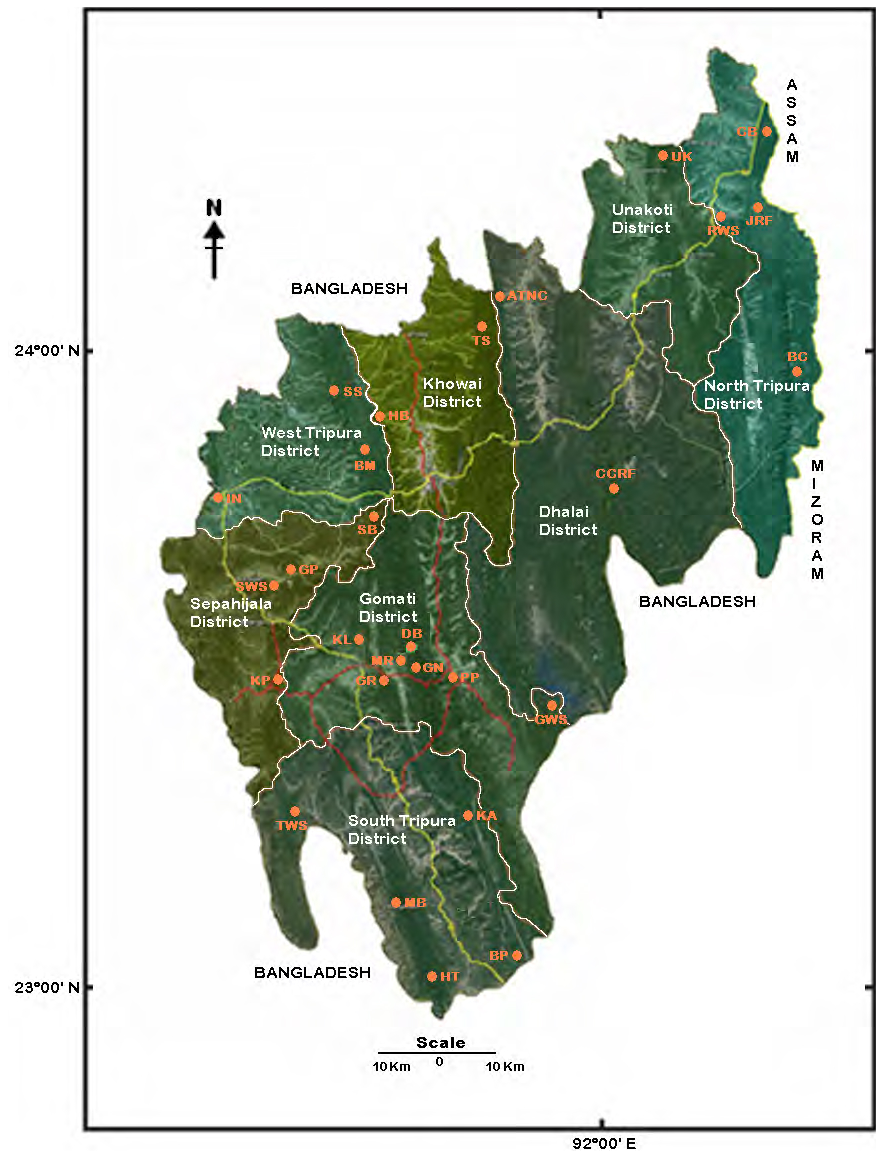

Figure 2. Google earth image of Tripura province, captured on 3 May 2013 , showing butterfly sampling sites denoted in red circle.

Table 1. Geo-coordinates, elevation and forest types of locations in different districts surveyed for butterflies in Tripura province, India.

\begin{tabular}{|c|c|c|c|c|c|c|}
\hline District & Location & $\begin{array}{l}\text { Abbreviation } \\
\text { of location }\end{array}$ & Latitude & Longitude & $\begin{array}{l}\text { Elevation } \\
\text { (m) }\end{array}$ & Forest type \\
\hline \multirow[t]{7}{*}{ Gomati } & Gumati Wild Life Sanctuary & GWS & $23^{\circ} 31^{\prime} 53.73^{\prime \prime} \mathrm{N}$ & $091^{\circ} 51^{\prime} 48.34^{\prime \prime} \mathrm{E}$ & 76 & Moist deciduous with moist bamboo brakes \\
\hline & Debbari & DB & $23^{\circ} 32^{\prime} 45.30^{\prime \prime} \mathrm{N}$ & $091^{\circ} 36^{\prime} 33.96^{\prime \prime} \mathrm{E}$ & 48 & Semi-evergreen \\
\hline & Killa & $\mathrm{KL}$ & $23^{\circ} 33^{\prime} 35.40^{\prime} \mathrm{N}$ & $091^{\circ} 30^{\prime} 06.00^{\prime \prime} \mathrm{E}$ & 70 & Moist mixed deciduous \\
\hline & Maharani & MR & $23^{\circ} 30^{\prime} 20.79^{\prime} \mathrm{N}$ & $091^{\circ} 34^{\prime} 16.49^{\prime \prime} \mathrm{E}$ & 30 & Moist mixed deciduous \\
\hline & Gandhari & GN & $23^{\circ} 29^{\prime} 44.46^{\prime} \mathrm{N}$ & $091^{\circ} 36^{\prime} 38.25^{\prime \prime} \mathrm{E}$ & 33 & Moist mixed deciduous \\
\hline & Garjee & GR & $23^{\circ} 28^{\prime} 59.10^{\prime \prime} \mathrm{N}$ & $091^{\circ} 31^{\prime} 59.36^{\prime \prime} \mathrm{E}$ & 30 & Moist mixed deciduous \\
\hline & Paharpur & PP & $23^{\circ} 29^{\prime} 30.94^{\prime} \mathrm{N}$ & $091^{\circ} 43^{\prime} 15.17^{\prime \prime} \mathrm{E}$ & 47 & Moist mixed deciduous \\
\hline \multirow[t]{5}{*}{ South } & Trishna Wild Life Sanctuary & TWS & $23^{\circ} 18^{\prime} 06.25^{\prime \prime} \mathrm{N}$ & $091^{\circ} 22^{\prime} 10.14^{\prime \prime} \mathrm{E}$ & 27 & Moist mixed deciduous \\
\hline & Kalshi & $\mathrm{KA}$ & $23^{\circ} 16^{\prime} 46.51^{\prime \prime} \mathrm{N}$ & $091^{\circ} 40^{\prime} 28.24^{\prime \prime} \mathrm{E}$ & 40 & Moist deciduous with moist bamboo brakes \\
\hline & Baisnabpur & $\mathrm{BP}$ & $23^{\circ} 01^{\prime} 46.84^{\prime \prime} \mathrm{N}$ & $091^{\circ} 44^{\prime} 47.87^{\prime \prime} \mathrm{E}$ & 29 & Moist mixed deciduous \\
\hline & Harbatoli & HT & $23^{\circ} 01^{\prime} 05.45^{\prime \prime} \mathrm{N}$ & $091^{\circ} 36^{\prime} 21.55^{\prime \prime} \mathrm{E}$ & 27 & Moist deciduous with moist bamboo brakes \\
\hline & Malumbari & MB & $23^{\circ} 08^{\prime} 17.46^{\prime \prime} \mathrm{N}$ & $091^{\circ} 32^{\prime} 53.34^{\prime \prime} \mathrm{E}$ & 15 & Moist deciduous with moist bamboo brakes \\
\hline \multirow[t]{4}{*}{ Sepahijala } & Sepahijala Wild Life Sanctuary & SWS & $23^{\circ} 39^{\prime} 26.94^{\prime \prime} \mathrm{N}$ & $091^{\circ} 18^{\prime} 47.81^{\prime \prime} \mathrm{E}$ & 45 & Moist deciduous with moist bamboo brakes \\
\hline & Gatirampara & GP & $23^{\circ} 40^{\prime} 23.68^{\prime \prime} \mathrm{N}$ & $091^{\circ} 20^{\prime} 55.44^{\prime \prime} \mathrm{E}$ & 48 & Open scrub forest \\
\hline & Kalapania & $\mathrm{KP}$ & $23^{\circ} 30^{\prime} 11.70^{\prime \prime} \mathrm{N}$ & $091^{\circ} 20^{\prime} 54.00^{\prime \prime} \mathrm{E}$ & 55 & Moist mixed deciduous with dry bamboo brakes \\
\hline & Sangumabari & SB & $23^{\circ} 43^{\prime} 34.07^{\prime \prime} \mathrm{N}$ & $091^{\circ} 32^{\prime} 12.88^{\prime \prime} \mathrm{E}$ & 47 & Moist mixed deciduous \\
\hline \multirow[t]{3}{*}{ West } & Baramura & BM & $23^{\circ} 50^{\prime} 55.24^{\prime \prime} \mathrm{N}$ & $091^{\circ} 31^{\prime} 29.95^{\prime \prime} \mathrm{E}$ & 122 & Moist mixed deciduous with dry bamboo brakes \\
\hline & Subalsingh & SS & $23^{\circ} 58^{\prime} 32.03^{\prime \prime} \mathrm{N}$ & $091^{\circ} 28^{\prime} 12.33^{\prime \prime} \mathrm{E}$ & 64 & Moist mixed deciduous with dry bamboo brakes \\
\hline & Ishanchandra Nagar & IN & $23^{\circ} 45^{\prime} 30.21^{\prime \prime} \mathrm{N}$ & $091^{\circ} 15^{\prime} 29.52^{\prime \prime} \mathrm{E}$ & 44 & Moist mixed deciduous with \\
\hline \multirow[t]{2}{*}{ Khowai } & Harbang & $\mathrm{HB}$ & $23^{\circ} 55^{\prime} 44.59^{\prime \prime} \mathrm{N}$ & $091^{\circ} 33^{\prime} 09.13^{\prime \prime} \mathrm{E}$ & 207 & Moist mixed deciduous \\
\hline & Tulashikar & TS & $24^{\circ} 01^{\prime} 57.77^{\prime \prime} \mathrm{N}$ & $091^{\circ} 42^{\prime} 47.73^{\prime \prime} \mathrm{E}$ & 67 & Semi-evergreen to mixed moist deciduous \\
\hline \multirow[t]{2}{*}{ Dhalai } & Atharamura North Circle & ATNC & $24^{\circ} 06^{\prime} 53.64^{\prime \prime} \mathrm{N}$ & $091^{\circ} 46^{\prime} 06.90^{\prime \prime} \mathrm{E}$ & 183 & Moist mixed deciduous with dry bamboo brakes \\
\hline & Central Catchment Reserve Forest & CCRF & $23^{\circ} 50^{\prime} 31.79^{\prime \prime} \mathrm{N}$ & $091^{\circ} 57^{\prime} 25.47^{\prime \prime} \mathrm{E}$ & 180 & Semi-evergreen to mixed moist deciduous \\
\hline Unakoti & Unakoti & UK & $24^{\circ} 20^{\prime} 10.20^{\prime \prime} \mathrm{N}$ & $092^{\circ} 03^{\prime} 13.59^{\prime \prime} \mathrm{E}$ & 115 & Semi-evergreen to mixed moist deciduous \\
\hline \multirow[t]{4}{*}{ North } & Rowa Wild Life Sanctuary & RWS & $24^{\circ} 12^{\prime} 02.36^{\prime \prime} \mathrm{N}$ & $092^{\circ} 05^{\prime} 54.12^{\prime \prime} \mathrm{E}$ & 40 & Moist mixed deciduous \\
\hline & Juri Reserve Forest & JRF & $24^{\circ} 12^{\prime} 42.99^{\prime \prime} \mathrm{N}$ & $092^{\circ} 12^{\prime} 44.58^{\prime \prime} \mathrm{E}$ & 100 & Moist mixed deciduous \\
\hline & Churaibari & $C B$ & $24^{\circ} 24^{\prime} 04.57^{\prime \prime} \mathrm{N}$ & $092^{\circ} 14^{\prime} 44.17^{\prime \prime} \mathrm{E}$ & 53 & Semi-evergreen to mixed moist deciduous \\
\hline & Ballianchip & $\mathrm{BC}$ & $23^{\circ} 59^{\prime} 46.86^{\prime \prime} \mathrm{N}$ & $092^{\circ} 16^{\prime} 50.91^{\prime \prime} \mathrm{E}$ & 565 & Semi-evergreen \\
\hline
\end{tabular}


by $<200$ individuals but $>100$ individuals, occasional represented by $<100$ individuals but $>50$ individuals, rare represented by $<50$ individuals but $>10$ individuals, and very rare represented by $<10$ individuals. Conservation status of butterfly species documented in this study are determined according to national and international conservation categories and compared with their abundance records for better understanding of threatened taxa across local and global habitats.

\section{RESULTS}

This communication is the first to present a systematic list of butterfly species from Tripura, India based on the current taxonomic classification of butterflies (Savela 2013). It documents butterfly species by recording their locations with GPS data, dates of collection, and number and sex of specimens examined or photographed, voucher numbers of collected specimens, and geographical distribution in India and elsewhere in the world (Kehimkar 2008). Species recorded include 142 species that are newly reported from Tripura (Agarwala et al.2010; Roy Choudhury et al. 2011; Majumder J et al. 2012b). Also included are 65 species enlisted under threatened categories by national law (Anonymous 1997, 2007) and by international conservation agencies (IUCN 2007; UNEP-WCMC 2011). Photographs of the species considered threatened are provided based on actual material examined (Figures 3-47).

In this study, we report 212 butterfly species which belonged to 128 genera in 24 subfamilies and six families (Table 2). The systematic list of butterfly species is organized by family, subfamily, and tribe with following information:

i. Valid scientific name of the species with authority and English name; scientific name as originally proposed along with publication data to the original description; names of species within a tribe are presented in alphabetical order.

ii. Specimen examined: Details of specimen(s) examined including the number of specimen(s) seen and their sex (male $\hat{O}$; female $O$; male or female $\hat{O} / P$ ), collection location using abbreviations as used in Table 1, date of collection, and voucher numbers (e.g., Pap.1. , denotes first female specimen of Papilionidae; Pap.4.dissi. $\widehat{\jmath}$ denotes fourth male specimen of Papilionidae of dissimilis form; Pap.5.img. $\hat{0} /$ + denotes image of the fifth male or female monomorphic specimen of Papilionidae), and name of the collector.

iii. Conservation status: Abundance record of each butterfly species from Tripura province categorized under very common, common, occasional, rare, and very rare as elaborated in the Materials and Methods section, and their conservation status at national scale as per Indian Wildlife Protection Act
(IWPA 1972: Schedule I, II and IV) or Red Data Book (Part 2) of India (Anonymous1997, 2007; Gupta and Mondal 2005), and at the global scale as per red lists of International Union for Conservation of Nature and Natural Resources (IUCN), or Convention on International Trade in Endangered Species of Wild Fauna and Flora (CITES), respectively (IUCN 2007; UNEP-WCMC 2011).

iv. Distribution records from states of India and elsewhere in the world: These include 142 species as new records from Tripura and are denoted by asterisks prefixing species names. In addition, family-wise records of number of subfamilies, genera and species showing the relative diversity of different taxa are presented in Table 2.

Table 2. Overview of taxonomic diversity, represented by numbers of subfamilies, genera and species of butterflies under different families, recorded in Tripura province.

\begin{tabular}{lccc}
\hline Family & $\begin{array}{c}\text { Number of } \\
\text { subfamilies }\end{array}$ & $\begin{array}{c}\text { Number of } \\
\text { genera }\end{array}$ & $\begin{array}{c}\text { Number of } \\
\text { species }\end{array}$ \\
\hline Papilionidae & $1(4.17 \%)$ & $7(5.46 \%)$ & $16(7.52 \%)$ \\
Nymphalidae & $12(50 \%)$ & $39(30.42 \%)$ & $79(37.13 \%)$ \\
Pieridae & $2(8.34 \%)$ & $12(9.36 \%)$ & $23(10.81 \%)$ \\
Lycaenidae & $5(20.85 \%)$ & $34(26.52 \%)$ & $50(23.50 \%)$ \\
Riodinidae & $1(4.17 \%)$ & $1(0.78 \%)$ & $1(0.47 \%)$ \\
Hesperiidae & $3(12.51 \%)$ & $35(27.30 \%)$ & $43(20.21 \%)$ \\
\hline Total & $24(100 \%)$ & $128(100 \%)$ & $212(100 \%)$ \\
\hline
\end{tabular}

\section{Systematic list of butterflies of the Tripura province}

Family Papilionidae

Subfamily Papilioninae

Tribe Troidini

1. *Atrophaneura varuna (White, 1842); Common Batwing Papilio varuna White, 1842, Notice of two new species of Papilio from Penang, presented to the British Museum by Sir. Wm. Norris, Entomologist 1: 280. TL: Penang, Malaysia.

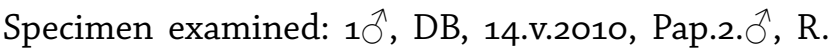
Lodh.

Conservation status: Tripura: very rare; IUCN/CITES: not evaluated.

Distribution: India (Uttaranchal in the west to Arunachal Pradesh in the east in Himalaya, Tripura), Bangladesh, Bhutan, Malaysia, Myanmar, Nepal.

2. *Byasa polyeuctes (Doubleday, 1842); Common Windmill Papilio polyeuctes Doubleday, 1842, in Gray, Zoological Miscellany 2: 74. TL: Sylhet, Bangladesh.

Specimen examined: 1ㅇ, BC, 18.viii.2009, Pap.1.우, D.K.Sinha.

Conservation status: Tripura: very rare; IUCN/CITES: not evaluated.

Distribution: India (Jammu and Kashmir in the west 
to Arunachal Pradesh in the east in Himalaya, Tripura), Bangladesh, Bhutan, Myanmar, Nepal, Pakistan.

\section{Pachliopta aristolochiae (Fabricius, 1775);}

\section{Common Rose}

Papilio aristolochiae Fabricius,1775, Systema Entomologiae, sistens insectorum classes, ordines, genera, species, adiectis synonymis, locis, descriptionibus, observationibus 1: 443. TL: Tranquebar, South India.

Specimen examined: $1 \hat{O}, \mathrm{BC}$, 18.viii.2009, Pap.3. $\hat{\circ}, \mathrm{R}$. Lodh; 1ㅇ, BC, 18.viii.2009, Pap.3.+ , R. Lodh.

Conservation status: Tripura: very common; India: IWPA/Red Date Book: not evaluated; IUCN/CITES: not evaluated.

Distribution: India (Common in almost all states, Tripura), Bangladesh, Bhutan, Myanmar, Nepal, Pakistan, Sri Lanka.

4. Troides helena (Linnaeus, 1758); Common Birdwing (Figure 3)

Papilio helena Linnaeus, 1758, Systema naturae per regna tria naturae, secundum classes, ordines, genera, species, cum characteribus, differentiis, synonymis, locis (Ed. 10) 1: 461. TL: Java.

Specimen examined: $1 \hat{0}$, BM, 15.x.2009, Pap.16. $\hat{0}, \mathrm{R}$. Lodh; 1ㅇ, BM, 15.x.2009, Pap.16.우, R. Lodh.

Conservation status: Tripura: common; CITES: Appendix II G.

Distribution: India (Arunachal Pradesh, Andaman and Nicobar Islands, Orissa, Sikkim, Tripura), America, Bangladesh, Bhutan, Myanmar, Nepal.

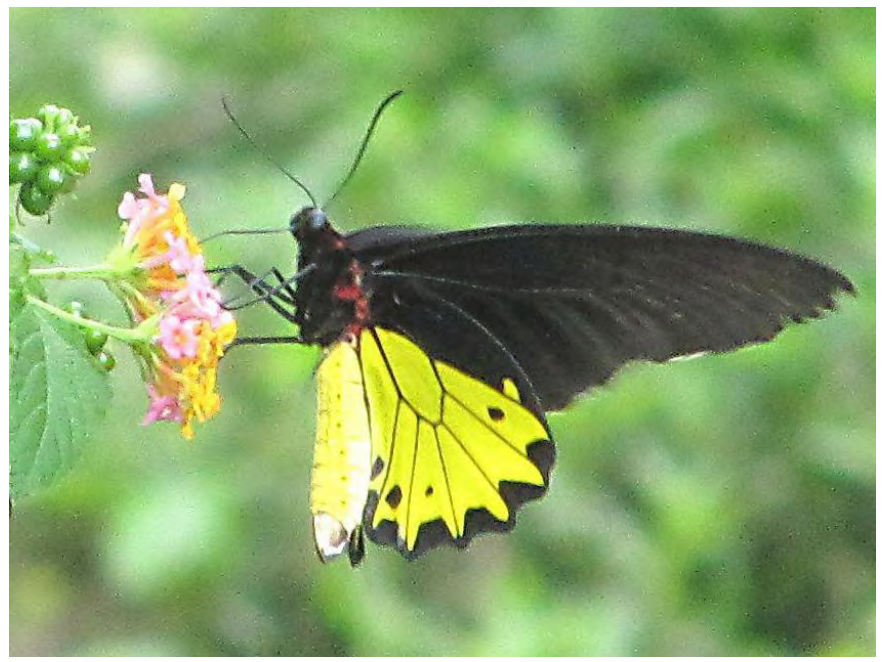

Figure 3. Troides helena.

Tribe Leptocircini

5. Graphium agamemnon (Linnaeus, 1758); Tailed Jay Papilio agamemnon Linnaeus, 1758 , Systema naturae per regna tria naturae, secundum classes, ordines, genera, species, cum characteribus, differentiis, synonymis, locis (Ed. 10) 1: 462. TL: China.

Specimen examined: 10 , TWS, 18.v.2010, Pap.7.img. $\overbrace{}^{\Uparrow}$, R. Lodh; 1우, TWS, 18.v.2010, Pap.7.img. 우, R. Lodh.

Conservation status: Tripura: rare; IUCN/CITES: not evaluated.
Distribution: India [Northeast, South India up to Gujarat (Kutch), Tripura, Uttaranchal in the west to Arunachal Pradesh in the east in Himalaya, West Bengal], Bangladesh, Bhutan, China, Nepal, Myanmar, Sri Lanka.

6. ${ }^{*}$ Graphium antiphates (Cramer, 1775); Five-bar Swordtail Papilio antiphates Cramer, 1775, Uitlandsche Kapellen (Papillons exotiques) 1 (1-7): 113, TL: South China.

Specimen examined: $1 \hat{0}$, DB, 29.vii.2009, Pap.8.img. $\hat{0}$, R. Lodh.

Conservation status: Tripura: rare; IUCN/CITES: not evaluated.

Distribution: India [Sikkim in the west to Arunachal Pradesh in the east in Himalaya, Tripura, Western Ghats (south of Goa)], Bangladesh, Bhutan, Nepal, Myanmar.

7. Graphium doson (C. Felder \& R. Felder, 1864);

\section{Common Jay}

Papilio doson C. Felder \& R. Felder, 1864, Verhandlungen der ZoologischBotanischen Gesellschaft in Wien 14(3): 305. TL: Sri Lanka.

Specimen examined: $1 \hat{\delta} / q$ (monomorphic), DB, 29. vii.2009, Pap.6.img. $\hat{\jmath} /$,, R. Lodh.

Conservation status: Tripura: rare; India: IWPA/Red Data Book: not evaluated; IUCN/CITES: not evaluated.

Distribution: India (Northeast, Orissa, South India up to Maharashtra, Tripura, Uttaranchal in the west to Arunachal Pradesh in the east in Himalaya, West Bengal), Bhutan, Nepal, Myanmar, Sri Lanka.

\section{Graphium sarpedon (Linnaeus, 1758);}

\section{Common Bluebottle}

Papilio sarpedon Linnaeus, 1758, Systema naturae per regna tria naturae, secundum classes, ordines, genera, species, cum characteribus, differentiis, synonymis, locis (Ed. 10) 1: 461. TL: Hainan, China.

Specimen examined: 1 \%/ $/$ (monomorphic), SS, 12. viii.2009, Pap.5.img. ${ }^{\lambda} /$,, R. Lodh.

Conservation status: Tripura: common; IUCN/CITES: not evaluated.

Distribution: India (Jammu and Kashmir in the west to Arunachal Pradesh in the east in Himalaya, Tripura), Bhutan, China, Myanmar, Nepal, Pakistan.

9. ${ }^{*}$ Lamproptera curius (Fabricius, 1787); White Dagontail Papilio curius Fabricius, 1787, Mantissa Insectorum 2: 9. TL: Thailand.

Specimens examined: $10^{\Uparrow}, \mathrm{DB}, 14 . \mathrm{v} \cdot 2010$, Pap.9. ${ }^{\lambda}, \mathrm{R}$. Lodh; 1, AMNC, 5.v.2012, Pap.9.ㅇ, R. Lodh.

Conservation status: Tripura: very rare; IUCN/CITES: not evaluated.

Distribution: India (Assam to Arunachal Pradesh in the east in Himalaya, Tripura), Bangladesh, Myanmar, Thailand.

Tribe Papilionini

10. ${ }^{*}$ Papilio castor Westwood, 1842 ; Common Raven Papilio castor Westwood, 1842, The Annals and Magazine of Natural History 9: 37. TL: Sylhet, Bangladesh. 
Specimen examined: 1우, RWS, 14.viii.2010, Pap.11.우, D.K. Sinha.

Conservation status: Tripura: very rare; IUCN/CITES: not evaluated.

Distribution: India (Northeast, Sikkim to Arunachal Pradesh in the east in Himalaya, Tripura), Bangladesh, Bhutan, Nepal, Myanmar.

\section{Papilio clytia Linnaeus, 1758; Common Mime} (Figure 4)

Papilio clytia Linnaeus, 1758, Systema naturae per regna tria naturae, secundum classes, ordines, genera, species, cum characteribus, differentiis, synonymis, locis (Ed. 10) 1: 479. TL: China.

Specimen examined: $10^{\lambda}$, AMNC, 20.ix.2009, Pap.4.

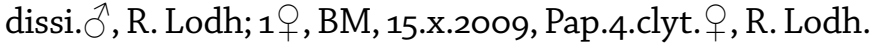

Conservation status: Tripura: rare; India: Schedule I (IWPA 1972), Endangered (Red Data Book); IUCN: Near Threatened.

Distribution: India (Himachal Pradesh in the west to Arunachal Pradesh in the east in Himalaya, South, central and eastern India, Tripura), Bangladesh, Bhutan, Myanmar, Nepal, Pakistan, Sri Lanka.

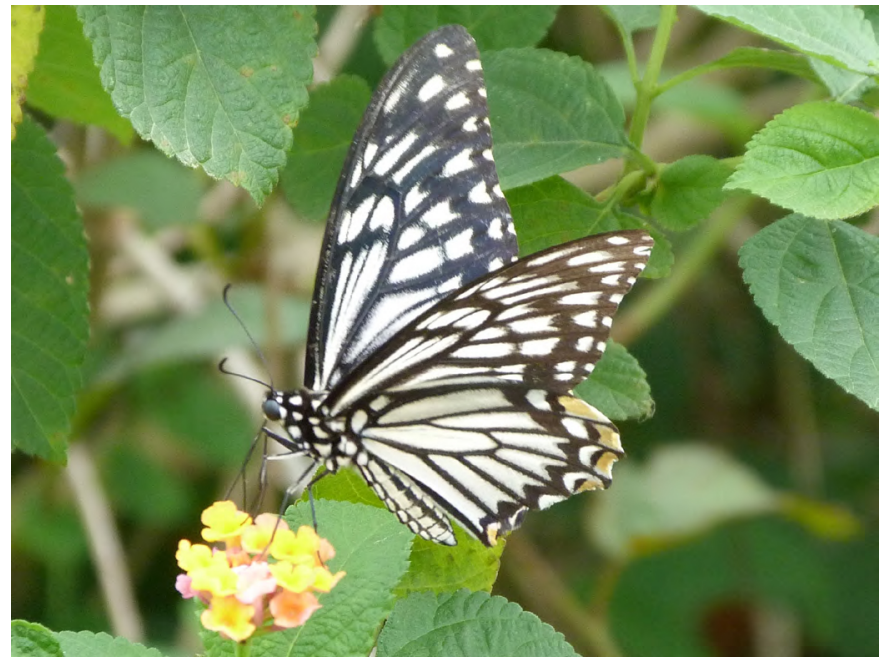

Figure 4. Papilio clytia.

12. Papilio demoleus Linnaeus, 1758; Lime Butterfly Papilio demoleus Linnaeus, 1758, Systema naturae per regna tria naturae, secundum classes, ordines, genera, species, cum characteribus, differentiis, synonymis, locis (Ed. 10) 1: 464. TL: Canton, China.

Specimen examined: 10, BC, 18.viii.2009, Pap.15. $0^{\lambda}$, R. Lodh; 1ㅇ, BC, 18.viii.2009, Pap.15. ㅇ, R. Lodh.

Conservation status: Tripura: very common; IUCN/ CITES: not evaluated.

Distribution: India (Common in almost all states of India, Tripura), Afghanistan, Bangladesh, Bhutan, China, Myanmar, Nepal, Pakistan, Sri Lanka.

13. *Papilio helenus Linnaeus, 1758; Red Helen

(Figure 5)

Papilio helenus Linnaeus, 1758, Systema naturae per regna tria naturae, secundum classes, ordines, genera, species, cum characteribus, differentiis, synonymis, locis (Ed. 10) 1: 459. TL: Canton, China.

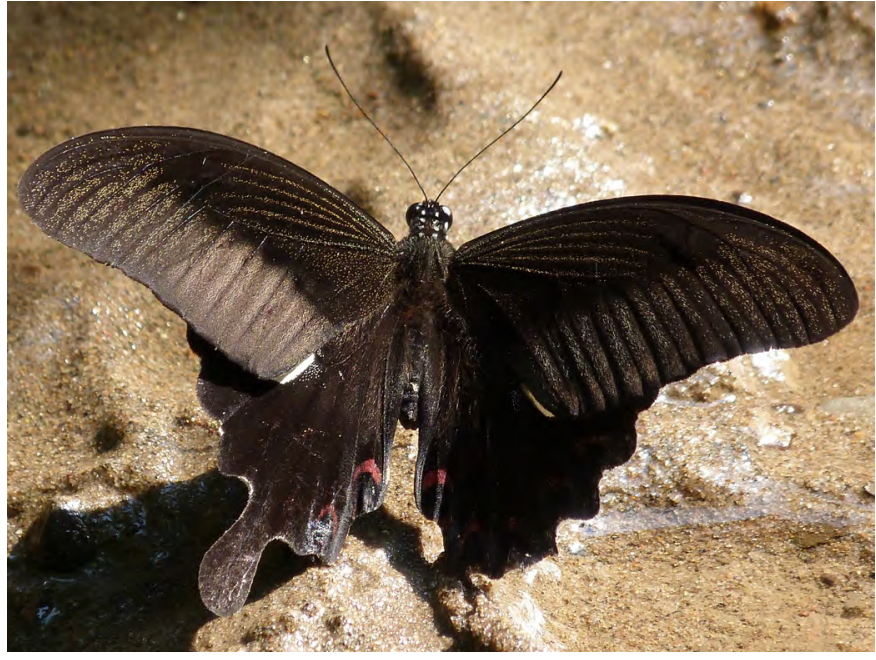

Figure 5. Papilio helenus.

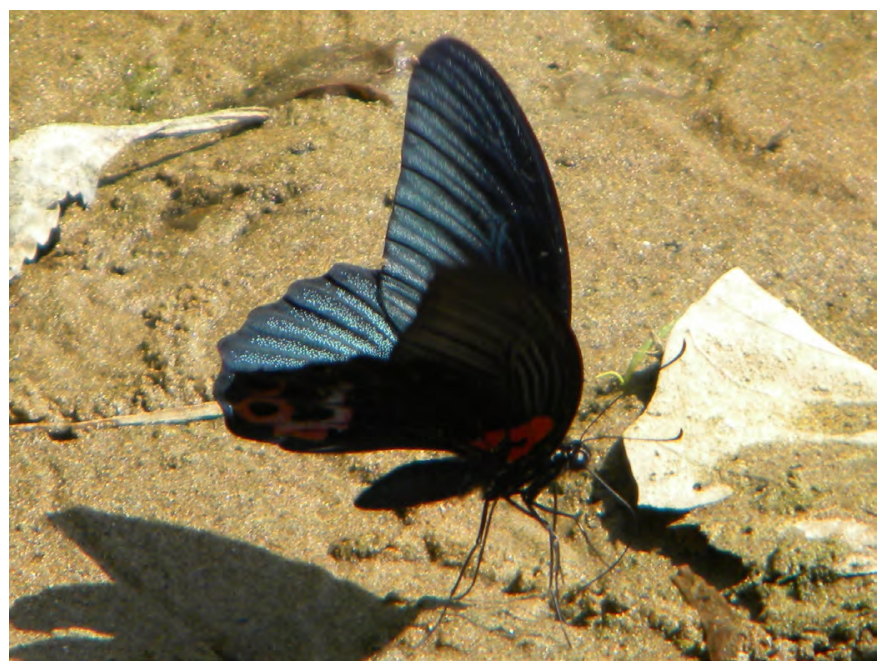

Figure 6. Papilio memnon.

Specimen examined: $1 \sigma^{\Uparrow} / \phi$, AMNC, 5.iv.2012, Pap.12. img $\hat{\partial} /$,, R. Lodh.

Conservation status: Tripura: rare; IUCN: Near Threatened.

Distribution: India (South India, Uttaranchal in the west to Arunachal Pradesh in the east in Himalaya, Tripura), Bangladesh, Bhutan, China, Myanmar, Nepal, Sri Lanka.

\section{Papilio memnon Linnaeus, 1758; Great Mormon}

(Figure 6)

Papilio memnon Linnaeus, 1758 , Systema naturae per regna tria naturae, secundum classes, ordines, genera, species, cum characteribus, differentiis, synonymis, locis (Ed. 10) 1: 460. TL: Asia.

Specimen examined: 10̂, KP, 9.viii.2009, Pap.14. agen. ${ }^{\Uparrow}$, R. Lodh; 1ㅇ, SS, 13.ii.2010, Pap.14.agen. + , R. Lodh; 1오, SS, 13.ii.2010, Pap.14.alca.우, R. Lodh.

Conservation status: Tripura: common; India: Schedule I (IWPA); IUCN/CITES: not evaluated.

Distribution: India (Arunachal Pradesh, Andaman and Nicobar Islands, Sikkim, Tripura, West Bengal), Bangladesh, Bhutan, Nepal, Myanmar. 
15. Papilio nephelus Boisduval, 1836; Yellow Helen Papilio nephelus Boisduval, 1836, Histoire naturelle des insectes, spécies général des lépidoptères 1: 210. TL: Java, Indonesia.

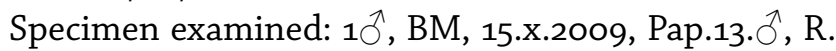
Lodh; 1 , , GR, 20.v.2010, Pap.13. 9 , R. Lodh.

Conservation status: Tripura: very common; India: Schedule II (IWPA); IUCN/CITES: not evaluated.

Distribution: India (Meghalaya, Orissa, Sikkim to Arunachal in the east in Himalaya, Tripura), Bangladesh, Bhutan, Indonesia, Myanmar, Nepal.

16. Papilio polytes Linnaeus, 1758; Common Mormon Papilio polytes Linnaeus, 1758, Systema naturae per regna tria naturae, secundum classes, ordines, genera, species, cum characteribus, differentiis, synonymis, locis (Ed. 10) 1: 46o. TL: South China.

Specimen examined: $1 \hat{0}$, TWS, 7.xi.2009, Pap.10. $\hat{\sigma}$, R. Lodh; 19 , SWS, 14.i.2010, Pap.10.stic. 9, R. Lodh; 1 , DB, 29.vii.2009, Pap.10.img.cyru. 9 , R. Lodh.

Conservation status: Tripura: very common; IUCN/ CITES: not evaluated.

Distribution: India (Common in almost all states of India, Tripura), Bangladesh, Bhutan, China, Nepal, Myanmar, Pakistan, Sri Lanka.

Family Nymphalidae

Subfamily Apaturinae

Tribe Apaturini

17. *Euripus nyctelius (Doubleday, 1845); Courtesan

Diadema nyctelius Doubleday, 1845, The Annals and Magazine of Natural History (1)16(104): 182. TL: Sylhet, Bangladesh.

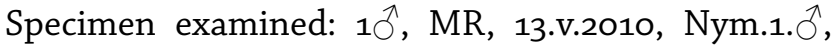
R. Lodh; 29 , MR, 13.v.2010, Nym.1.i. + , Nym.1.ii. + , R. Lodh.

Conservation status: Tripura: rare; IUCN/CITES: not evaluated.

Distribution: India (Arunachal Pradesh, Assam, Sikkim, Tripura), Bangladesh, Myanmar.

18. *Rohana parisatis (Westwood, 1850); Black Prince Apatura parisatis Westwood, 1850, The genera of diurnal Lepidoptera, comprising their generic characters, a notice of their habitats and transformations, and a catalogue of the species of each genus 2: 305. TL: Assam, India.

Specimen examined: $1 \hat{\jmath}, \mathrm{DB}$, 7.iv.2010, Nym.2. $\hat{\sigma}, \mathrm{R}$. Lodh.

Conservation status: Tripura: very rare; IUCN/CITES: not evaluated.

Distribution: India (Northeast, South India up to Gujarat, Tripura, Uttaranchal in the west to Arunachal Pradesh in the east in Himalaya), Bangladesh, Myanmar, Sri Lanka.

Subfamily Biblidinae

Tribe Biblidini

19. Ariadne ariadne (Linnaeus, 1763); Angled Castor Papilio ariadne Linnaeus, 1763, in Johansson (Thesis), Centuria
Insectorum, Amoenitates Academicae 6: 407. TL: Java, Indonesia.

Specimen examined: $1 \overbrace{}^{\lambda}, \mathrm{MR}, 13 \cdot \mathrm{v} \cdot 2010, \mathrm{Nym} \cdot 3 \cdot \overbrace{}^{\top}, \mathrm{R}$. Lodh.

Conservation status: Tripura: very common; IUCN/ CITES: not evaluated.

Distribution: India (common except in arid North and Northeast, Tripura), Bangladesh, Bhutan, Indonesia, Myanmar, Nepal, Sri Lanka.

\section{Ariadne merione (Cramer, 1777); Common Castor}

\section{(Figure 7)}

Papilio merione Cramer, 1777, Uitlandsche Kapellen (Papillons exotiques) 2(9-16): 76. TL: Coromandel, South India.

Specimen examined: $1 \hat{\sigma}, \mathrm{MR}, 13 \cdot \mathrm{v} \cdot 2010, \mathrm{Nym} \cdot 4 \cdot \hat{\sigma}, \mathrm{R}$. Lodh.

Conservation status: Tripura: occasional; IUCN: Near Threatened.

Distribution: India (common except in arid Northwest, Tripura), Bangladesh, Bhutan, Myanmar, Nepal, Pakistan, Sri Lanka.

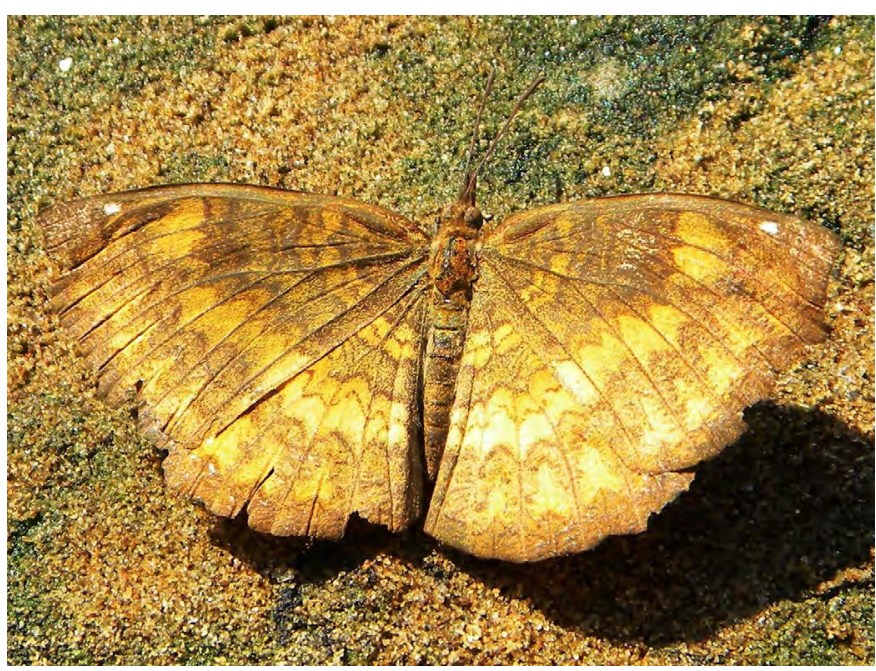

Figure 7. Ariadne merione.

Subfamily Charaxinae

Tribe Charaxini

21. * Charaxes athamas (Drury, 1773); Common Nawab (Figure 8)

Papilio athamas Drury, 1773, Illustrations of natural history, Exotic Insects 1: 5. TL: China.

Specimen examined: $1 \hat{\jmath}, \mathrm{KL}$, 9.x.2009, Nym.7. $\hat{\sigma}, \mathrm{R}$. Lodh.

Conservation status: Tripura: rare; India: Schedule II (IWPA); IUCN: Near Threatened.

Distribution: India (Andaman, Northeast, peninsular India, Tripura, Uttaranchal in the west to Arunachal Pradesh in the east in Himalaya), Bangladesh, Bhutan, Myanmar, Nepal, Pakistan, Sri Lanka. 


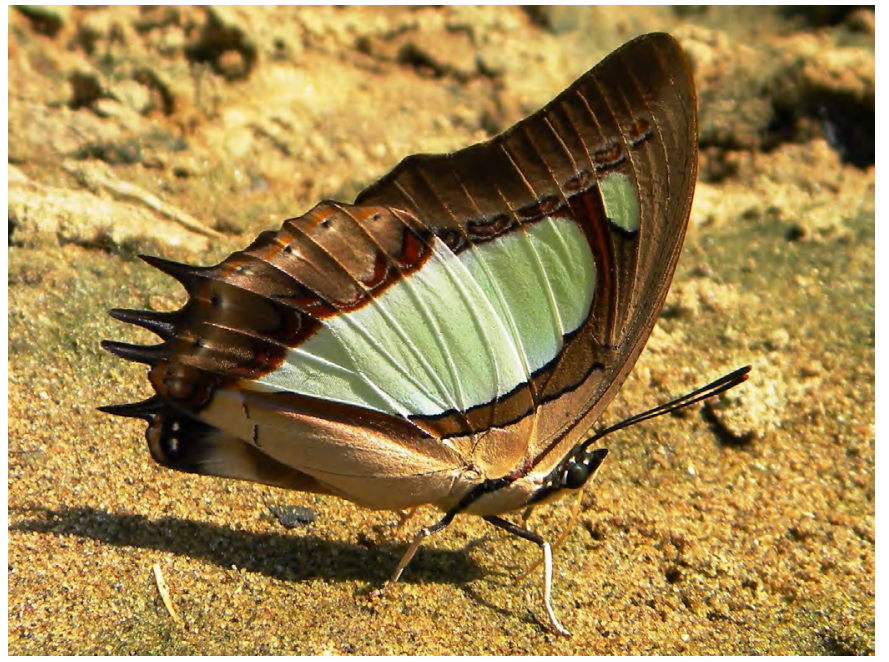

Figure 8. Charaxes athamas.

22. *Charaxes bernardus (Fabricius, 1793); Tawny Rajah Papilio bernardus Fabricius, 1793, Entomologia systematica 3 (1): 71. TL: China.

Specimen examined: $1 \hat{\jmath} / q$ (monomorphic), RWS, 18.viii.2009, Nym.5.img. $\lesssim / Q$, R. Lodh.

Conservation status: Tripura: very rare; India: Schedule II (IWPA).

Distribution: India (Bihar, South India, Tripura, Uttaranchal in the west to Northeast in the east in Himalaya), Bangladesh, Bhutan, Myanmar, Nepal.

23. *Charaxes delphis Doubleday, 1843;

Jewelled Nawab

Charaxes delphis Doubleday, 1843, Annales de la Société Entomologique de France (2)1(3): 217. TL: Sylhet, Bangladesh.

Specimen examined: $1 \hat{\sigma} / \phi$ (monomorphic), DB, 7.iv.2010, Nym.8.img. $\lesssim / O$, R. Lodh.

Conservation status: Tripura: rare; IUCN/CITES: not evaluated.

Distribution: India (Northeast, Sikkim in the west to Arunachal Pradesh in the east in Himalaya, Tripura), Bangladesh, Myanmar.

24. *Charaxes solon (Fabricius, 1793); Black Rajah Papilio solon Fabricius, 1793, Entomologia systematica 3 (1): 69. TL: Tranquebar, South India.

Specimen examined: $1 \hat{\jmath}$, CCRF, 20.viii.2009, Nym.6. $\sigma^{\Uparrow}$, R. Lodh.

Conservation status: Tripura: very rare; India: Schedule II (IWPA); IUCN: Near Threatened.

Distribution: India (common except in extreme Northwest, Tripura), Bangladesh, Bhutan, Myanmar, Nepal, Sri Lanka.

Subfamily Cyrestinae

Tribe Cyrestini

25. *hersonesia risa (Doubleday, 1848);

Common Maplet
Cyrestis risa Doubleday, [1848], The genera of diurnal Lepidoptera, comprising their generic characters, a notice of their habitats and transformations, and a catalogue of the species of each genus 2: 32. TL: Assam, India.

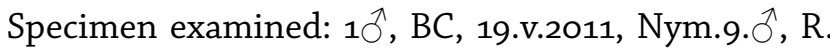
Lodh.

Conservation status: Tripura: rare; IUCN/CITES: not evaluated.

Distribution: India (Northeast, Tripura, Uttaranchal in the west to Arunachal Pradesh in the east in Himalaya), Bangladesh, Bhutan, Myanmar, Nepal.

\section{6. *Cyrestis cocles (Fabricius, 1787); Marbled Map}

(Figure 9)

Papilio cocles Fabricius, 1787, Mantissa Insectorum 2: 7 TL: Andaman, India.

Specimen examined: $1{ }^{\lambda}, B C, 19 . v \cdot 2011$, Nym.10. ${ }^{\lambda}, \mathrm{R}$. Lodh.

Conservation status: Tripura: very rare; India: Schedule II (IWPA).

Distribution: India [Eastern Ghats (Jharkhand, Orissa), Arunachal Pradesh, Assam, Sikkim, Tripura], Bhutan, Myanmar.

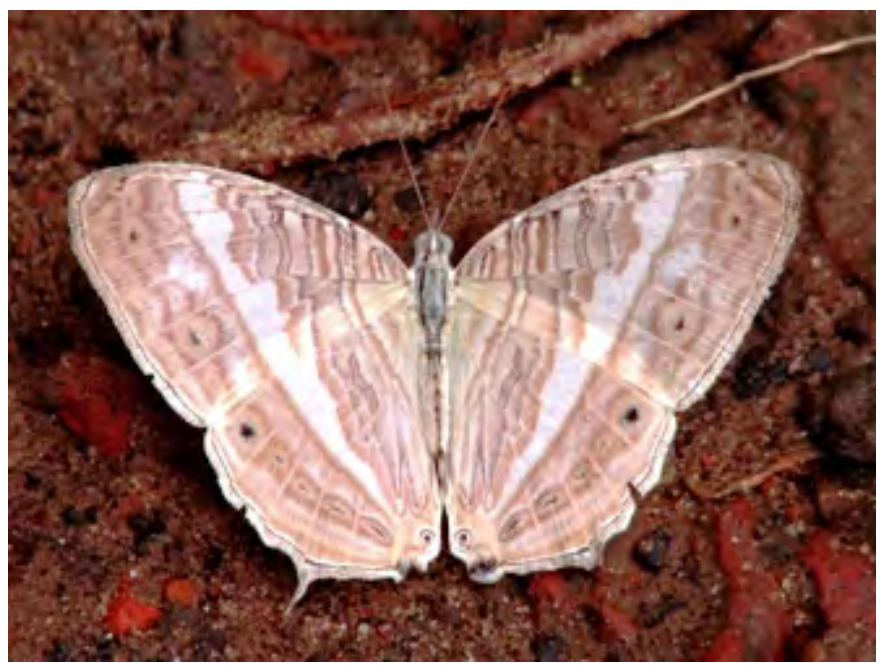

Figure 9. Cyrestis cocles.

Subfamily Pseudergolinae

Tribe Pseudergolini

27. *Stibochiona nicea (Gray, 1846); Popinjay

Adolias nicea Gray, 1846, Descriptions and Figures of some new Lepidopterous Insects chiefly from Nepal: 13. TL: Nepal.

Specimen examined: 10 , GWS, 12.xi.2009, Nym.11.a. ${ }^{\Uparrow}$, R. Lodh.

Conservation status: Tripura: rare; IUCN/CITES: not evaluated.

Distribution: India (Himachal Pradesh in the west to Arunachal Pradesh in the east in Himalaya, Northeast, Tripura), Bangladesh, Bhutan, Myanmar, Nepal.

Subfamily Danainae 
Tribe Danaini

28. Danaus chrysippus (Linnaeus, 1758); Plain Tiger Papilio chrysippus Linnaeus, 1758, Systema naturae per regna tria naturae, secundum classes, ordines, genera, species, cum characteribus, differentiis, synonymis, locis (Ed. 10) 1: 471. TL: China, Canton.

Specimen examined: $1 \hat{\jmath}$, KL, 9.X.2009, Nym.13. $0^{\lambda}, \mathrm{R}$. Lodh; 1ㅇ, KL, 9.x.2009, Nym.13.+, R. Lodh.

Conservation status: Tripura: rare; IUCN/CITES: not evaluated.

Distribution: India (common in almost all states of India, Tripura), Afghanistan, Bangladesh, Bhutan, China, Myanmar, Nepal, Pakistan, Sri Lanka.

\section{Danaus genutia (Cramer, 1779); Striped Tiger}

Papilio genutia Cramer, 1779, Uitlandsche Kapellen (Papillons exotiques) 3 (17-21): 23. TL: China, Canton.

Specimen examined: 10 $0^{\lambda}$ BM, 15.x.2009, Nym.12. $0^{\lambda}$, R. Lodh; 1ㅇ, BM, 15.x.2009, Nym.12. 9 , R. Lodh.

Conservation status: Tripura: common; IUCN/CITES: not evaluated.

Distribution: India (common in almost all states of India, Tripura), Bangladesh, Bhutan, China, Myanmar, Nepal, Pakistan, Sri Lanka.

30. ${ }^{*}$ Danaus melanippus (Cramer, 1777); White Tiger Papilio melanippus Cramer, 1777, Uitlandsche Kapellen (Papillons exotiques) 2 (9-16): 44. TL: Java, Indonesia.

Specimen examined: $1 \hat{\delta} / \phi$ (monomorphic), HT, 10.xi.2009, Nym.14.img. $\hat{\jmath} / 9$, R. Lodh.

Conservation status: Tripura: very rare; IUCN/CITES: not evaluated.

Distribution: India (Orissa, Tripura, West Bengal up to Arunachal Pradesh in the east in Himalaya), Bangladesh, Indonesia, Myanmar.

\section{Euploea core (Cramer, 1780); Common Crow} Papilio core Cramer, 1780, Uitlandsche Kapellen (Papillons exotiques) 3 (23-24): 133. TL: east coast, India.

Specimen examined: $1{ }^{\lambda}$, SWS, 6.viii.2009, Nym.15. ${ }^{\lambda}$, R. Lodh; 1오, SWS, 6.viii.2009, Nym.15.9, R. Lodh.

Conservation status: Tripura: very common; IUCN/ CITES: not evaluated.

Distribution: India (common in almost all states of India, Tripura), Bangladesh, Bhutan, Myanmar, Nepal, Sri Lanka.

32. *Euploea klugii Moore and Horsefield, 1857;

Brown King Crow

Euploea klugii Moore and Horsfield, 1857, in Horsfield and Moore, A Catalogue of the lepidopterous insects in the museum of the Honourable East-India Company 1: 130. TL: Assam, Cherrapunji, India.

Specimen examined: $1 \hat{\delta} / q$ (monomorphic), MR, 13.v.2010, Nym.18.img. ${ }^{\Uparrow} / q$, R. Lodh.

Conservation status: Tripura: very rare; near threatened (IUCN).

Distribution: India (Arunachal Pradesh, Orissa, Sikkim,
South India up to Maharashtra, Tripura, West Bengal), Bhutan, Myanmar, Nepal, Sri Lanka.

\section{3. ${ }^{*}$ Euploea midamus (Linnaeus, 1758);}

Blue Spotted Crow (Figure 10)

Papilio midamus Linnaeus, 1758, Systema naturae per regna tria naturae, secundum classes, ordines, genera, species, cum characteribus, differentiis, synonymis, locis (Ed. 10) 1: 470. TL: Canton, China.

Specimen examined: $10^{\lambda}, \mathrm{BP}, 11 . x i .2009$, Nym.17. $\overbrace{}^{\lambda}, \mathrm{R}$. Lodh; 1, BP, 11.xi.2009, Nym.17. + , R. Lodh.

Conservation status: Tripura: occasional; India: Schedule I (IWPA).

Distribution: India (Himachal Pradesh in the west to Arunachal Pradesh in the east in Himalaya, Tripura), Bangladesh, Bhutan, China, Myanmar, Nepal.

\section{4. ${ }^{*}$ Euploea mulciber (Cramer, 1777);}

Striped Blue Crow (Figure 11)

Papilio mulciber Cramer, 1777, Uitlandsche Kapellen (Papillons exotiques) 2 (9-16): 45. TL: east coast of South India.

Specimen examined: $1 \hat{0}$, GR, 5.xi.2009, Nym.16. $0^{\lambda}, \mathrm{R}$. Lodh; 1, GR, 5.xi.2009, Nym.16., , R. Lodh.

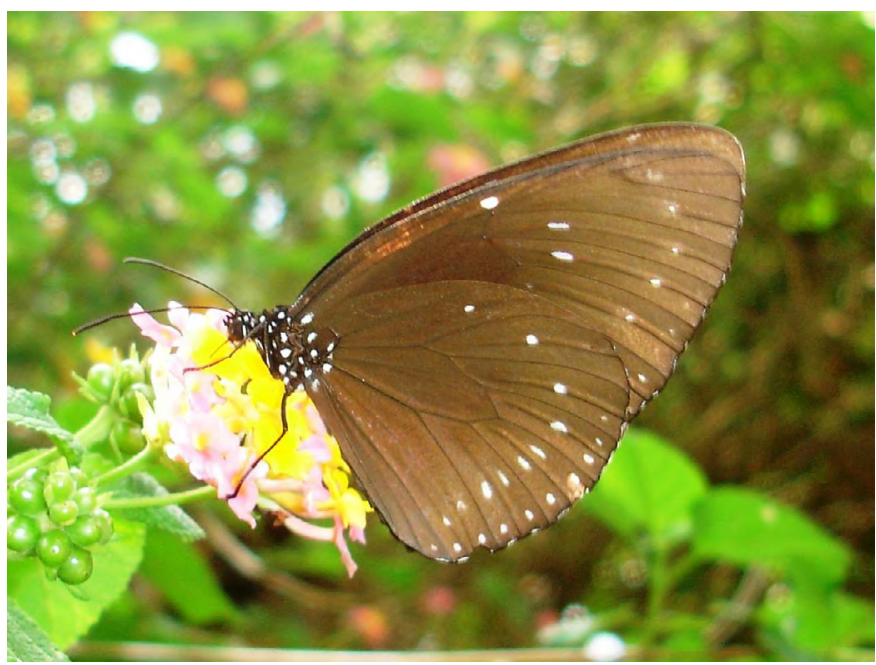

Figure 10. Euploea midamus.

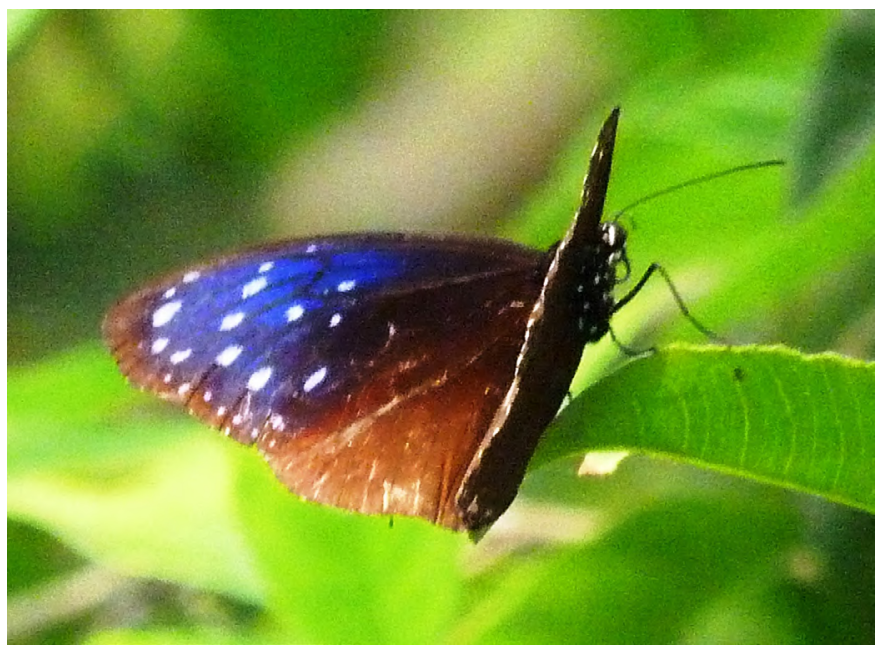

Figure 11. Euploea mulciber. 
Conservation status: Tripura: occasional; India: Schedule IV (IWPA); IUCN/CITES: not evaluated.

Distribution: India (Eastern Ghats, Himachal Pradesh in the west to Arunachal Pradesh in the east in Himalaya, Northeast, Tripura), Bangladesh, Bhutan, Myanmar, Nepal.

35. *Euploea radamanthus (Fabricius, 1793);

Magpie Crow

Papilio radamanthus Fabricius, 1793, Entomologia systematica 3(1): 42. TL: Asia.

Specimen examined: $1 \hat{\delta} \not+$ (monomorphic), MR, 13.v.2010, Nym.19.img. $\lesssim / q$, R. Lodh.

Conservation status: Tripura: very rare; not evaluated.

Distribution: India (Arunachal Pradesh, Northeast, Sikkim, Tripura), Bangladesh, Bhutan, China, Myanmar, Nepal.

36. Parantica aglea (Stoll, 1781); Glassy Tiger

Papilio aglea Stoll, 1781, Uitlandsche Kapellen (Papillons exotiques) 4 (32-32): 173. TL: Sri Lanka.

Specimen examined: 2 , $\mathrm{KL}, 8 . x i .2009$, Nym.20.i. + , Nym.20.ii. + , R. Lodh.

Conservation status: Tripura: occasional; IUCN/ CITES: not evaluated.

Distribution: India (common in almost all states of India, Tripura), Bangladesh, Bhutan, Myanmar, Nepal, Sri Lanka.

37. *Tirumala limniace (Cramer, 1775); Blue Tiger

Papilio limniace Cramer, 1775, Uitlandsche Kapellen (Papillons exotiques) 1(1-7): 92. TL: China.

Specimen examined:2 $q$, GWS, 12.xi.2009, Nym.21.i. $ᄋ$, Nym.21.ii. + , R. Lodh.

Conservation status: Tripura: occasional; IUCN/ CITES: not evaluated.

Distribution: India (common in almost all states of India, Tripura), Afghanistan, Bangladesh, Bhutan, China, Myanmar, Nepal, Pakistan, Sri Lanka.

Subfamily Heliconiinae

Tribe Acraeini

38. *Acraea violae (Fabricius, 1775); Tawny Coster Papilio violae Fabricius, 1775, Entomologia systematica 3: 460. TL: Tranquebar, South India.

Specimen examined: 1 +, HT, 10.xi.2009, Nym.22. , R. Lodh.

Conservation status: Tripura: very rare; IUCN/CITES: not evaluated.

Distribution: India (common in almost all states of India, Tripura), Bangladesh, Bhutan, Nepal, Pakistan, Sri Lanka.

39. *Cethosia biblis (Drury, 1770); Red Lacewing Papilio biblis Drury, 1770, Illustrations of natural history, Exotic Insec 1: 9. TL: Assam, India; China.
Specimen examined: 1ð̂, BC, 19.v.2011, Nym.23. img. $̄$, R. Lodh.

Conservation status: Tripura: very rare; IUCN/CITES: not evaluated.

Distribution: India (Andaman and Nicobar Island, Arunachal Pradesh, Assam, Sikkim, Himalaya, Tripura) Bangladesh, Bhutan, Myanmar, Nepal.

40. *Cethosia cyane (Drury, 1770); Leopard Lacewing Papilio cyane Drury, 1770, Illustrations of natural history, Exotic Insects 1: 4. TL: Bengal, India.

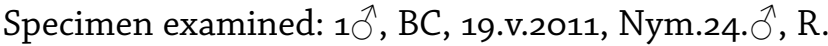
Lodh.

Conservation status: Tripura: rare; IUCN/CITES: not evaluated.

Distribution: India [Northeast, Orissa (Eastern Ghats), Tripura, Uttaranchal in the west to Arunachal Pradesh in the east in Himalaya, West Bengal], Bangladesh, Bhutan, Myanmar, Nepal.

Tribe Vagrantini

41. * Cirrochroa tyche C. Felder \& R. Felder, 1861;

Common Yeoman

Cirrochroa tyche C. Felder \& R. Felder, 1861, Wiener entomologische Monatschrift 5(10): 301. TL: Mindoro, Philippines.

Specimen examined: $1 \hat{\jmath}, \mathrm{HT}, 10 . x i .2009$, Nym.25. $\overbrace{}^{\dagger}$, R. Lodh.

Conservation status: Tripura: rare; IUCN/CITES: not evaluated.

Distribution: India (Andaman, Northeast, Arunachal Pradesh, Assam, Sikkim, Tripura, West Bengal), Bangladesh, Bhutan, Myanmar, Nepal, Philippines.

42. *Cupha erymanthis (Drury, 1773); Rustic

Papilio erymanthis Drury, 1773, Illustrations of natural history, Exotic Insects 1: 29. TL: Sumatra, Borneo, Indonesia.

Specimen examined: 1 , DB, 7.iv.2010, Nym.26. + , R. Lodh.

Conservation status: Tripura: very rare; IUCN: Near Threatened.

Distribution: India (Andaman and Nicobar Islands, Northeast, South India up to Maharashtra, Tripura, Uttranchal in the west to Arunachal Pradesh in the east in Himalaya), Bangladesh, Bhutan, Myanmar, Nepal, Sri Lanka.

43. Phalanta phalantha (Drury, 1773);

Common Leopard

Papilio phalantha Drury, 1773, Illustrations of natural history, Exotic Insect 1: 41. TL: Madras or Bombay, India.

Specimen examined: $1 \widehat{\jmath}, \mathrm{MB}, 16 . x \mathrm{ii.2009}, \mathrm{Nym} .27 . \overbrace{}^{\Uparrow}$, R. Lodh; 1 q, SWS, 6.viii.2009, Nym.27. 9 , R. Lodh.

Conservation status: Tripura: rare; IUCN/CITES: not evaluated.

Distribution: India (common in almost all states of India, Tripura), Bangladesh, Myanmar, Pakistan, Sri Lanka. 
44. ${ }^{*}$ Vindula erota (Fabricius, 1793); Cruiser

(Figure 12)

Papilio erota Fabricius, 1793, Entomologia systematica 3(1): 76. TL: Pulau Salang, South Thailand.

Specimen examined: $10^{\lambda}, B C, 19 . v \cdot 2011$, Nym.28. ${ }^{\lambda}, \mathrm{R}$. Lodh.

Conservation status: Tripura: rare; IUCN: Near Threatened.

Distribution: India (Andaman, Arunachal Pradesh, Northeast, Sikkim, Tripura, Western Ghats), Bangladesh, Bhutan, Myanmar, Nepal, Thailand.

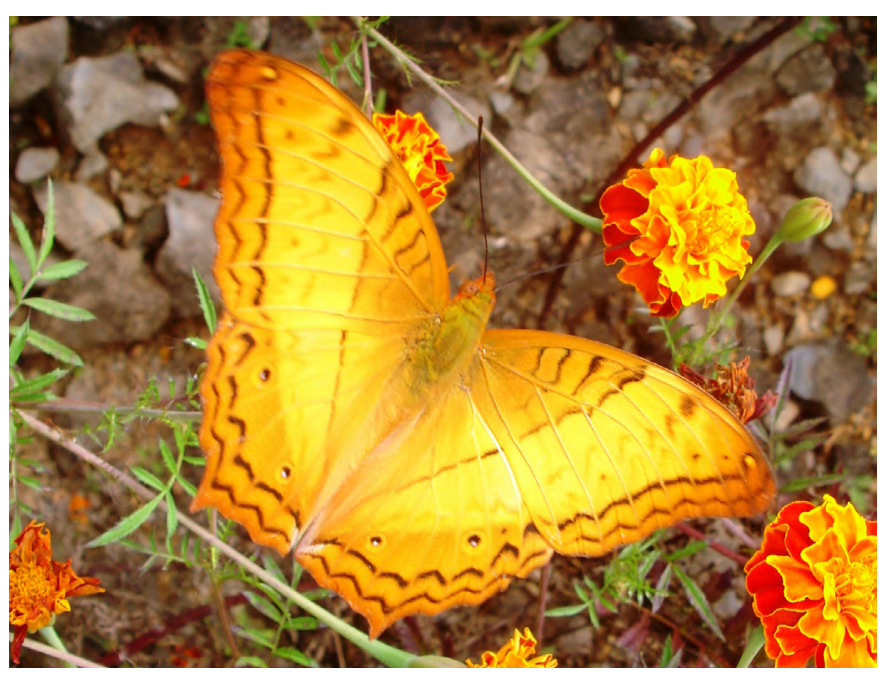

Figure 12. Vindula erota.

Subfamily Libytheinae

45. *Libythea myrrha Godart, 1819; Club Beak

(Figure 13)

Libythea myrrha Godart, 1819, Encyclopédie méthodique 9(1): 169, 171. TL: Java, Indonesia; Bombay, India.

Specimen examined: $1 \hat{\delta} / 9$ (monomorphic), DB, 29.vii.2009, Nym.29img. $\delta /$, R. Lodh.

Conservation status: Tripura: very rare; IUCN: Near Threatened.

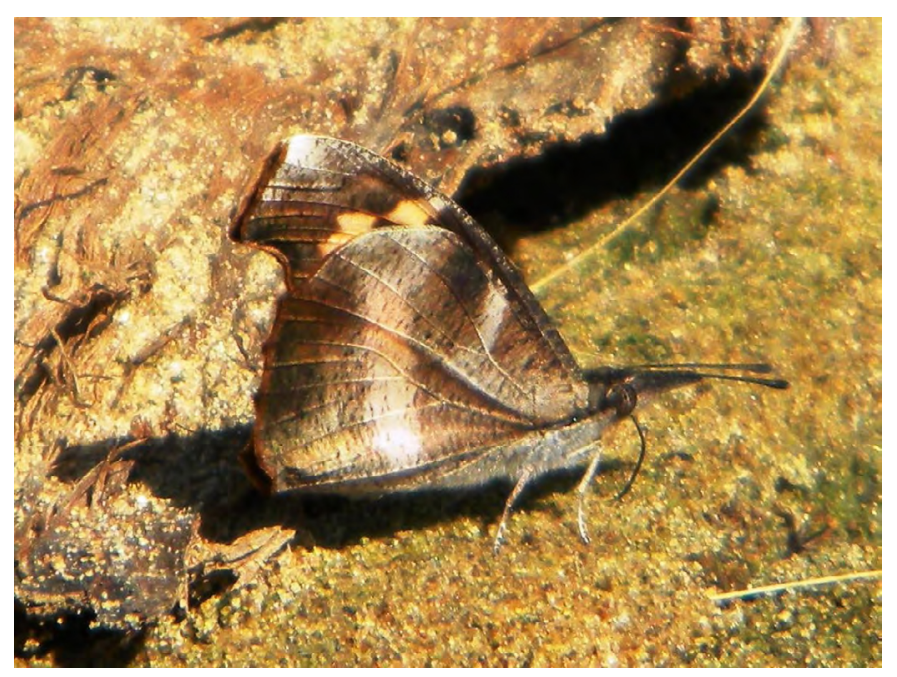

Figure 13. Libythea myrrha.
Distribution: India (Himachal Pradesh in the west to Arunachal Pradesh in the east in Himalaya, Northeast, South India, Tripura,), Afghanistan, Bangladesh, Bhutan, Indonesia, Myanmar, Nepal, Pakistan, Sri Lanka.

Subfamily Limenitidinae

Tribe Limenitidini

46. *Athyma asura Moore, 1857; Studded Sergeant Athyma asura Moore, 1857, in Horsfield and Moore, A Catalogue of the lepidopterous insects in the museum of the Honourable East-India Company 1: 171. TL: India.

Specimen examined: $1 \delta / q$ (monomorphic), DB, 7.iv.2010, Nym.31.img. ${ }^{\top} / Q$, R. Lodh.

Conservation status: Tripura: very rare; India: Schedule II (IWPA).

Distribution: India (Arunachal Pradesh, Assam, Himachal Pradesh, Tripura), Bangladesh, Bhutan, Nepal, Myanmar.

47. Athyma perius (Linnaeus, 1758); Common Sergeant Papilio perius Linnaeus, 1758, Systema naturae per regna tria naturae, secundum classes, ordines, genera, species, cum characteribus, differentiis, synonymis, locis (Ed. 10) 1: 471. TL: India.

Specimen examined: $1{ }^{\lambda}$, SWS, 6.viii.2009, Nym.30. $0^{\lambda}$, R. Lodh.

Conservation status: Tripura: common; IUCN/CITES: not evaluated.

Distribution: India (Himachal Pradesh in the west to Arunachal Pradesh in the east in Himalaya, Northeast, South India, Madhya Pradesh, Tripura), Myanmar.

48. *Athyma nefte (Cramer, 1782); Colour Sergeant Papilio nefte Cramer, 1782, Uitlandsche Kapellen (Papillons exotiques) 3(22): 111. TL: Java, Indonesia.

Specimen examined: $1 \hat{0}$, KL, 9.X.2009, Nym.33. $0^{\lambda}, \mathrm{R}$. Lodh; 1우, SWS, 6.viii.2009, Nym.33.ㅇ, R. Lodh.

Conservation status: Tripura: occasional; IUCN/ CITES: not evaluated.

Distribution: India (Andaman, Arunachal Pradesh, Northeast, Orissa, Sikkim, South India, Tripura), Bangladesh, Bhutan, Indonesia, Myanmar, Nepal.

49. *Athyma ranga Moore, 1857; Black Vein Sergeant (Figure 14)

Athyma ranga Moore 1857, in Horsfield and Moore, A Catalogue of the lepidopterous insects in the museum of the Honourable East-India Company 1: 175. TL: Sikkim, India.

Specimen examined: $1 \delta / \%$ (monomorphic), DB, 7.iv.2010, Nym.32.img. $\hat{\jmath} /$,, R. Lodh.

Conservation status: Tripura: very rare; India: Schedule II (IWPA).

Distribution: India [Arunachal Pradesh, Northeast, Sikkim, Tripura, Western Ghats (Karnataka southwards)], Bangladesh, Bhutan, Myanmar, Nepal. 


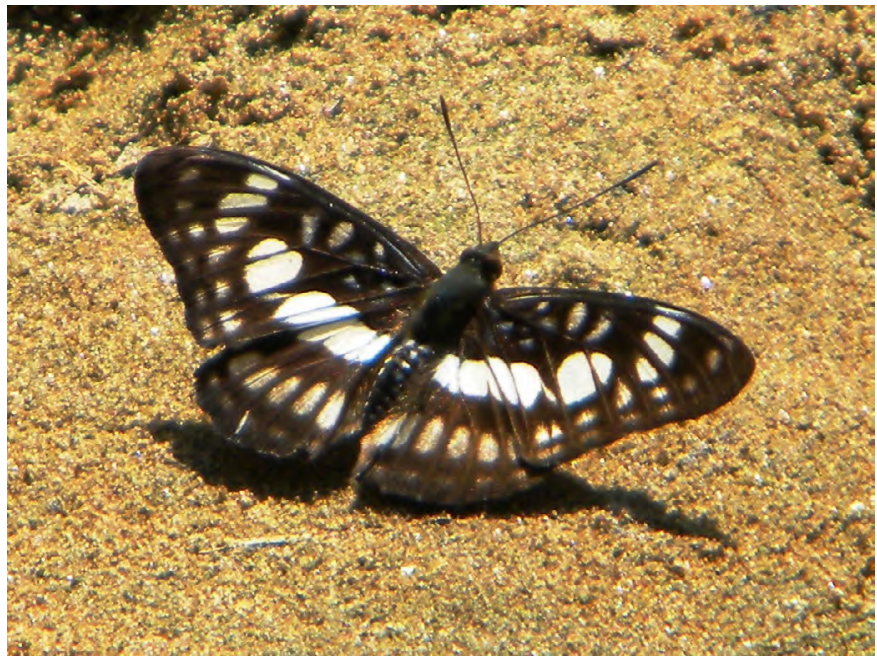

Figure 14. Athyma ranga.

50. Lebadea martha (Fabricius, 1787); Knight

Papilio martha Fabricius, 1787, Mantissa Insectorum 2: 56. TL: Pulau Salang, Phuket, Thailand.

Specimen examined: 1, BM, 15.x.2009, Nym.38.우, R. Lodh.

Conservation status: Tripura: rare; IUCN/CITES: not evaluated.

Distribution: India [Arunachal Pradesh, Northeast, Sikkim, Tripura, West Bengal (Darjeeling-Siliguri region)], Bangladesh, Bhutan, Myanmar, Nepal, Thailand.

\section{Moduza procris (Cramer, 1779); Commander}

Papilio procris Cramer, 1779, Uitlandsche Kapellen (Papillons exotiques) 2(9-16): 15. TL: Java, Indonesia.

Specimen examined: $1 \sigma^{\lambda}, \mathrm{MB}, 16 . x i 1.2009$, Nym.40. ${ }^{\lambda}$, R. Lodh.

Conservation status: Tripura: rare; IUCN/CITES: not evaluated.

Distribution: India (Andaman, Northeast, South India, Madhya Pradesh, Tripura, Uttaranchal, West Bengal), Bangladesh, Indonesia, Myanmar, Sri Lanka.

Tribe Adoliadini

52. *Dophla evelina (Stoll, 1790); Red Spot Duke Papilio evelina Stoll, 1790, Aanhangsel van het Werk, de Uitlandsche Kapellen Aanhangsel Werk (2-5): 132. TL: Coromandel Coast, India.

Specimen examined: $1 \hat{\sigma} / \phi$ (monomorphic), DB, 14.v.2010, Nym.34.img. $\hat{\jmath} / q$, R. Lodh.

Conservation status: Tripura: very rare; IUCN/CITES: not evaluated.

Distribution: India (Northeast, South India, Maharashtra, Tripura), Bangladesh, Myanmar, Sri Lanka.

53. Euthalia aconthea (Cramer, 1779); Common Baron (Figure 15)

Papilio aconthea Cramer, 1779, Uitlandsche Kapellen (Papillons exotiques) 2(9-16): 59. TL: Java, Indonsia.

Specimen examined: $1 \hat{\jmath}, \mathrm{MR}, 16 . x \mathrm{ii.2009}, \mathrm{Nym} .36 .{ }^{\lambda}$, R. Lodh; 19, MR, 16.xii.2009, Nym.36. ㅇ, R. Lodh.

Conservation status: Tripura: occasional; India: Schedule II (IWPA).

Distribution: India (common in almost all states of India, Tripura), Bangladesh, Bhutan, Indonesia, Myanmar, Nepal, Pakistan, Sri Lanka.

54. *Euthalia lubentina (Cramer, 1779); Gaudy Baron

(Figure 16)

Papilio lubentina Cramer, 1779, Uitlandsche Kapellen (Papillons exotiques) 2(9-16): 92. TL: South China.

Specimen examined: 19 , AMNC, 17.i.2010, Nym.37. img. , , R. Lodh.

Conservation status: Tripura: very rare; India: Schedule IV (IWPA), IUCN: Endangered.

Distribution: India (Himachal Pradesh in the west to Arunachal Pradesh in the east in Himalaya, Northeast, South India, West Bengal, Tripura), Bangladesh, Bhutan, China, Myanmar, Nepal, Sri Lanka.

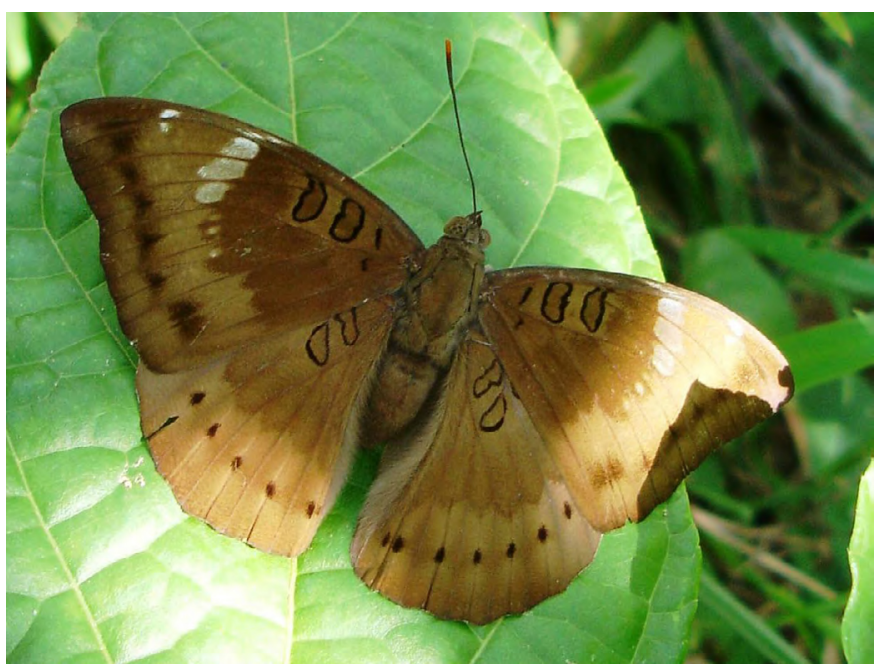

Figure 15. Euthalia aconthea.

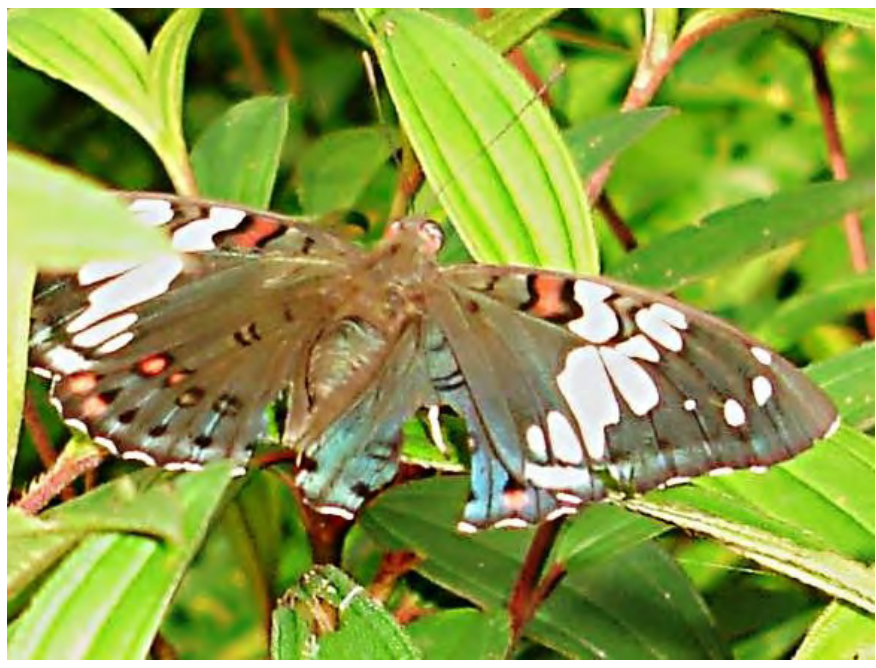

Figure 16. Euthalia lubentina. 
55. *Euthalia monina (Fabricius, 1787);

Powdered Baron

Papilio monina Fabricius, 1787, Mantissa Insectorum 2: 51. TL: West Malaysia.

Specimen examined: $10^{\lambda} / q$ (monomorphic), TWS, 18.v.2010, Nym.35.img. $\sigma^{\top} /$, R. Lodh.

Conservation status: Tripura: very rare; IUCN/CITES: not evaluated.

Distribution: India (Arunachal Pradesh, Northeast, Sikkim, Tripura), Bangladesh, Malaysia, Myanmar.

\section{6. ${ }^{*}$ Lexias dirtea (Fabricius, 1793); Dark Archduke}

\section{(Figure 17)}

Papilio dirtea Fabricius, 1793, Entomologia systematica 3(1): 59. TL: Naga Hills, India; Myanmar.

Specimen examined: 10, DB, 7.iv.2010. Nym.39. img. ${ }^{\top}$. R. Lodh.

Conservation status: Tripura: very rare; India: Schedule II (IWPA).

Distribution: India (Northeast, Sikkim, Tripura), Bangladesh, Bhutan, Myanmar.

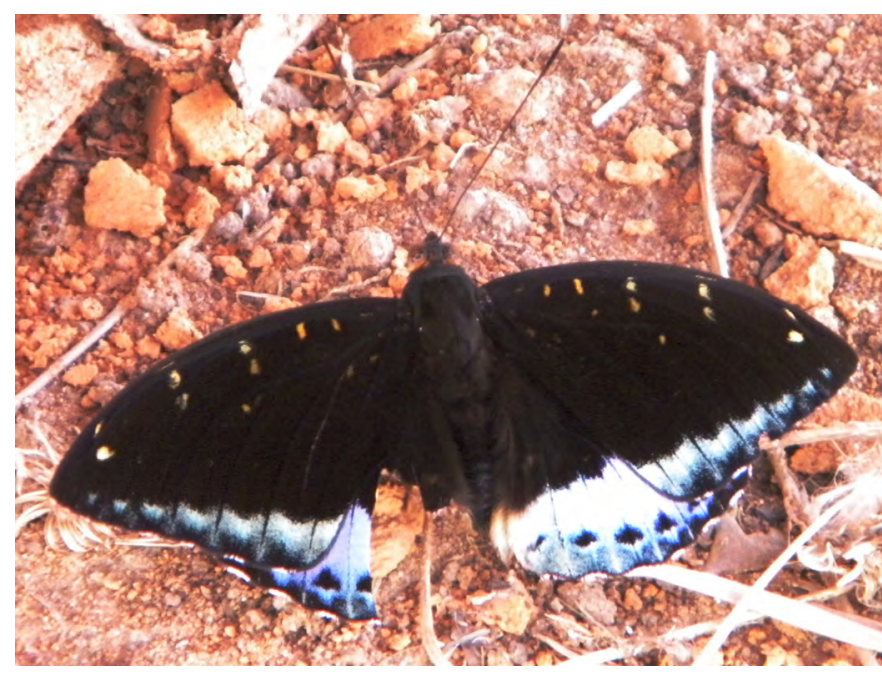

Figure 17. Lexias dirtea.

57. ${ }^{*}$ Tanaecia julii (Lesson, 1837); Common Earl

Nymula julii Lesson, 1837, in Bougainville, Journal de la navigation autour du globe, de la frégate La Thétis et de la corvette L'Espérance, Voyage Thetis 2: 345. TL: Sumatra, Indonesia.

Specimen examined: 10 $\hat{0}$, PP, 3.vi.2009, Nym.49. img. ${ }^{\lambda}$, R. Lodh.

Conservation status: Tripura: occasional; IUCN/ CITES: not evaluated.

Distribution: India (Northeast, Tripura, Uttranchal in the west to Arunachal Pradesh in the east in Himalaya), Bangladesh, Bhutan, Indonesia, Myanmar, Nepal.

\section{Tanaecia lepidea (Butler, 1868); Grey Count}

(Figure 18)

Adolias lepidea Butler, 1868, The Annals and Magazine of Natural History (4)1: 71. TL: Assam, India.

Specimen examined: $1 \hat{\jmath}$, SWS, 6.viii.2009, Nym.48. ${ }^{\lambda}$, R. Lodh; 1오, SWS, 6.viii.2009, Nym.48. , R. Lodh.

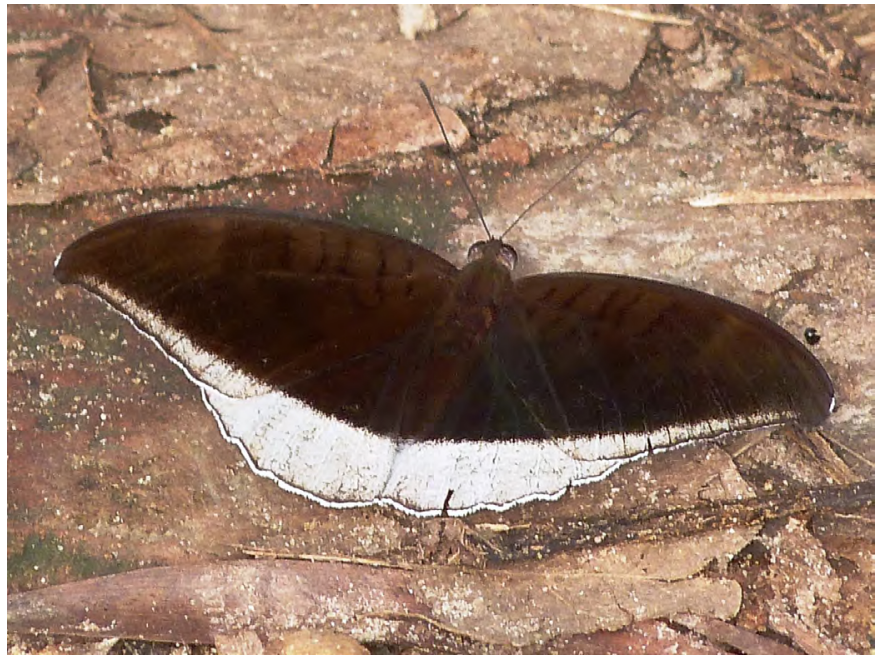

Figure 18. Tanaecia lepidea.

Conservation status: Tripura: common; India: Schedule II (IWPA).

Distribution: India (Madhya Pradesh and Karnataka southwards, Northeast, Orissa, South India up to Maharashtra, Tripura, Uttranchal in the west to Arunachal Pradesh in the east in Himalaya), Bangladesh, Bhutan, Myanmar, Nepal.

Tribe Neptini

59. ${ }^{*}$ Neptis clinia Moore, 1872 ; Clear Sailor

Neptis clinia Moore, 1872, Proceedings of the Zoological Society of London (2): 563. TL: Bengal, India.

Specimen examined: $1 \hat{\delta} / \phi$ (monomorphic), DB, 7.iv.2010, Nym.43.img. ${ }^{\top} / q$, R. Lodh.

Conservation status: Tripura: very rare; IUCN/CITES: not evaluated.

Distribution: India [Andaman, Arunachal Pradesh, Madhya Pradesh, Northeast, Sikkim, South India (Goa southwards), Tripura], Bhutan, Myanmar, Nepal.

6o. *Neptis harita Moore, 1874; Dingiest Sailor

Neptis harita Moore 1874, Proceedings of the Zoological Society of London (4): 571. TL: East Bengal, India.

Specimen examined: $1 \hat{\delta} / \phi$ (monomorphic), DB, 7.iv.2010, Nym.45.img. ${ }^{\wedge} / Q$, R. Lodh.

Conservation status: Tripura: very rare; IUCN/CITES: not evaluated.

Distribution: India (Assam, Bengal, Tripura), Myanmar.

61. Neptis hylas (Linnaeus, 1758); Common Sailor

Papilio hylas Linnaeus, 1758, Systema naturae per regna tria naturae, secundum classes, ordines, genera, species, cum characteribus, differentiis, synonymis, locis (Ed. 10) 1: 486. TL: China; India.

Specimen examined: 10 , KP, 9.viiii.2009, Nym.42. $\overbrace{}^{\lambda}$, R. Lodh; 19, KP, 9.viii.i2009, Nym.42. 9 , R. Lodh.

Conservation status: Tripura: very common; IUCN/ CITES: not evaluated.

Distribution: India (common in almost all states of 
India, Tripura), Bangladesh, Bhutan, Nepal, Pakistan, Sri Lanka.

62. *Neptis jumbah Moore, 1857;

Chestnut-Streaked Sailor (Figure 19)

Neptis jumbah Moore, 1857, in Horsfield and Moore, A Catalogue of the lepidopterous insects in the museum of the Honourable East-India Company 1: 167. TL: Sri Lanka.

Specimen examined: 1 $\widehat{\delta} / \phi$ (monomorphic), DB, 7.iv.2010, Nym.41.img. $\overbrace{}^{\Uparrow} / q$, R. Lodh.

Conservation status: Tripura: very rare; IUCN/CITES: Schedule I (IWPA).

Distribution: India (Andaman, Arunachal Pradesh, Madhya Pradesh, Sikkim, South India, Tripura, West Bengal), Bangladesh, Myanmar, Sri Lanka.

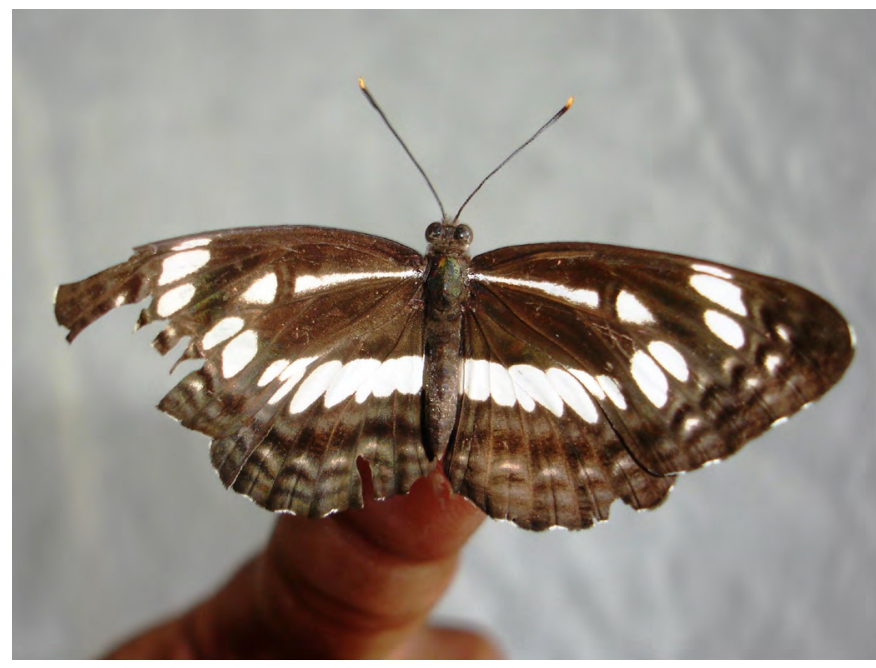

Figure 19. Neptis jumbah.

63. *Neptis soma Moore, 1858; Sullied Sailor

Neptis soma Moore, 1858, Proceedings of the Zoological Society of London 26: 9. TL: Sylhet, Bangladesh; North India.

Specimen examined: $1 \hat{\delta} / q$ (monomorphic), DB, 7.iv.2010, Nym.44.img. $\hat{\delta} / \phi$, R. Lodh.

Conservation status: Tripura: very rare; India: Schedule II (IWPA).

Distribution: India [Andaman, Jammu and Kashmir in the west to Arunachal Pradesh in the east in Himalaya, Madhya Pradesh, South India (Western Ghats, other hills and up to Karnataka, Tripura], Bhutan, Myanmar, Nepal.

64. Pantoporia hordonia (Stoll, 1790); Common Lascar (Figure 20)

Papilio hordonia Stoll, 179o, Aanhangsel van het werk, De uitlandsche kapellen (2-5): 149. TL: "Guinea Coast, Africa" [error for Bengal].

Specimen examined: $10^{\hat{\alpha}}$, GWS, 12.xi.2009, Nym.46.a.

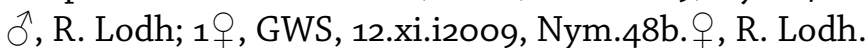

Conservation status: Tripura: occasional; IUCN: Near Threatened.

Distribution: India (Northeast, South India, Tripura, Uttaranchal in the west to Arunachal Pradesh in the east

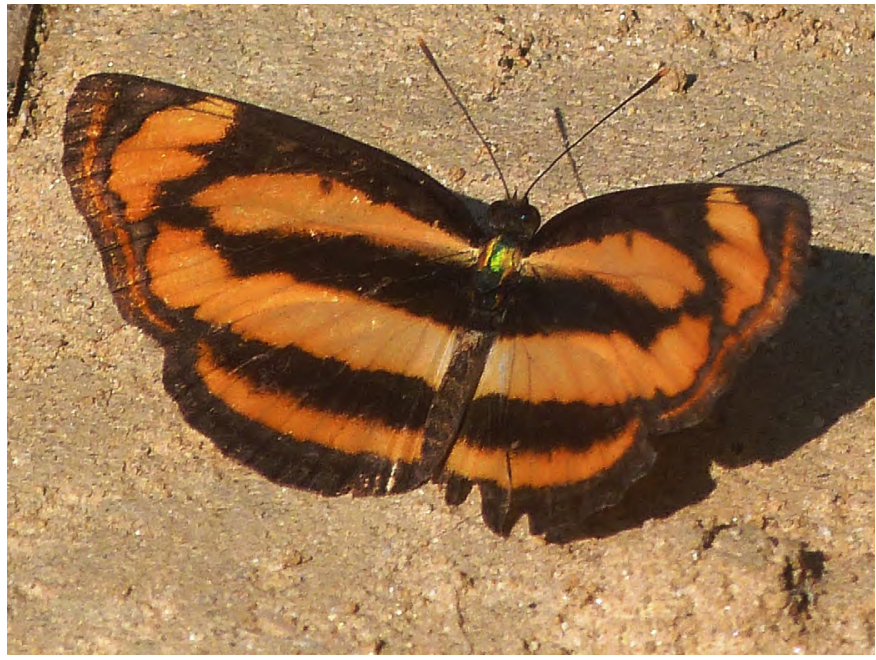

Figure 20. Pantoporia hordonia.

in Himalaya, Tripura), Bangladesh, Bhutan, Myanmar, Nepal, Sri Lanka.

Tribe Parthenini

\section{5. ${ }^{*}$ Parthenos sylvia (Cramer, 1776); Clipper}

\section{(Figure 21)}

Papilio sylvia Cramer, 1776, Uitlandsche Kapellen (Papillons exotiques) 1 (1-7): 68. TL: Java, Indonesia.

Specimen examined: $1{ }^{\lambda}$, SWS, 6.viii.2009, Nym.47. $0^{\lambda}$, R. Lodh.

Conservation status: Tripura: occasional; India: Schedule II (IWPA), IUCN: Near Threatened.

Distribution: India [Andaman and Nicobar Islands, Northeast, South India (Western and Eastern Ghats), Tripura, Uttaranchal in the west to Arunachal Pradesh in the east in Himalaya], Bangladesh, Indonesia, Myanmar, Sri Lanka,

Subfamily Morphinae

Tribe Amathusiini

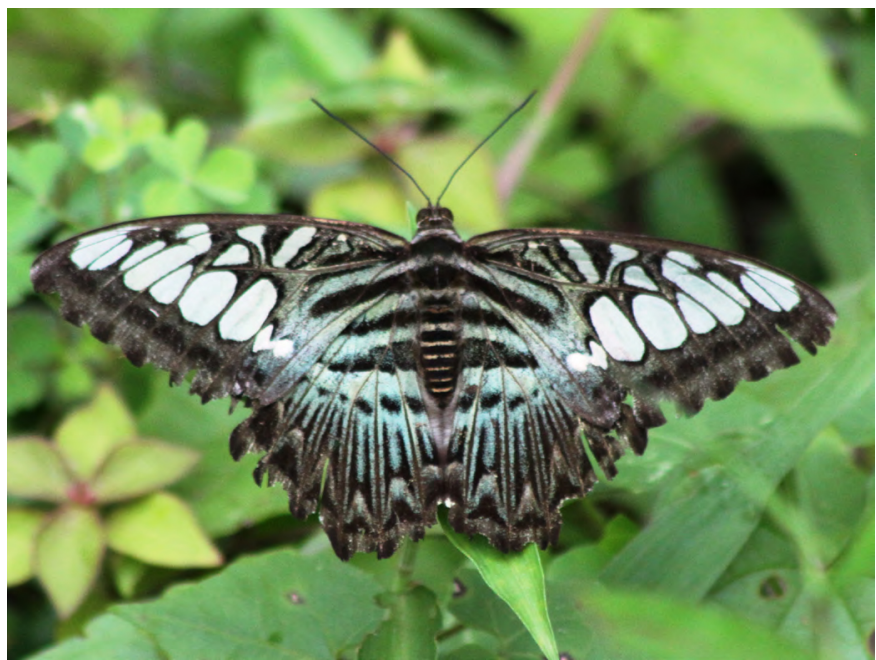

Figue 21. Parthenos sylvia. 
66. *Discophora sondaica Boisduval, 1836;

Common Duffer

Discophora sondaica Boisduval, 1836, Histoire naturelle des insectes. Species général des lépidoptéres 1: 12. TL: Java, Indonesia.

Specimen examined: $1 \hat{0}$, GWS, 12.xi.2009, Nym.51. $\hat{0}$, R. Lodh.

Conservation status: Tripura: rare; India: Schedule I (IWPA), IUCN: Critically Endangered.

Distribution: India (Arunachal Pradesh, Northeast, Sikkim, Tripura, West Bengal), Bangladesh, Bhutan, Nepal, Myanmar.

67. Discophora timora Westwood, 1850; Great Duffer (Figure 22)

Discophora timora Westwood, [1850], The genera of diurnal Lepidoptera, comprising their generic characters, a notice of their habitats and transformations, and a catalogue of the species of each genus (2): 54. TL: Sylhet, Bangladesh.

Specimen examined: $1 \hat{0}, C B, 17 . v i i i .2009$, Nym.50. $0^{\lambda}$, R. Lodh.

Conservation status: Tripura: very rare; India: Schedule II (IWPA).

Distribution: India (Arunachal Pradesh, Northeast, Sikkim, Tripura, West Bengal), Bangladesh, Bhutan, Myanmar.

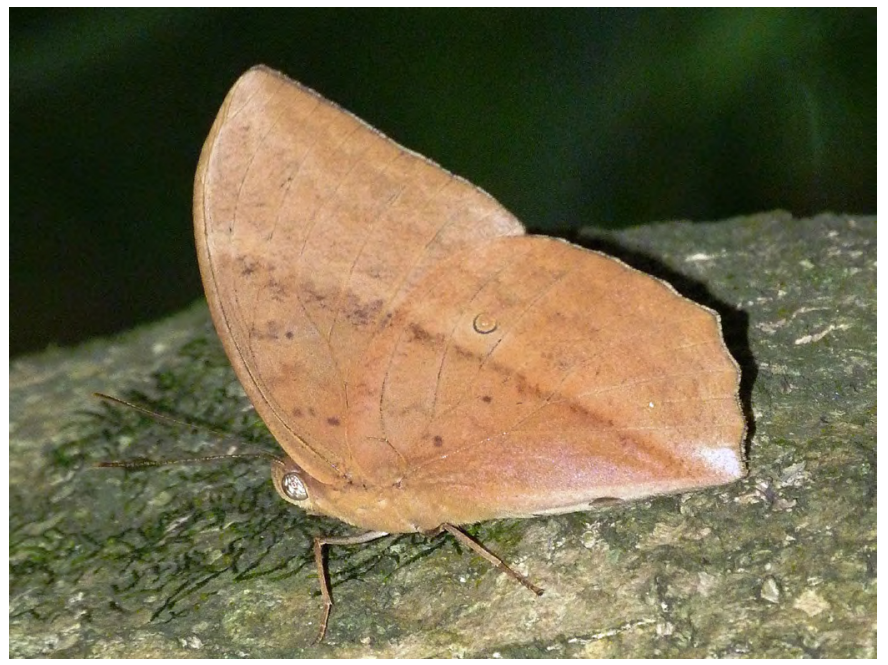

Figure 22. Discophora timora.

Subfamily Nymphalinae

Tribe Junoniini

68. Hypolimnas bolina (Linnaeus, 1758); Great Egg fly Papilio bolina Linnaeus, 1758, Systema naturae per regna tria naturae, secundum classes, ordines, genera, species, cum characteribus, differentiis, synonymis, locis (Ed. 10) 1: 479. TL: India.

Specimen examined: 10 , DB, 14.v.2010, Nym.52. $0^{\lambda}, \mathrm{R}$. Lodh; 10 , AMNC, 5.v.2012, Nym.52. ㅇ, R. Lodh.

Conservation status: Tripura: rare; IUCN/CITES: not evaluated.

Distribution: India (common in almost all states of India, Tripura), Bangladesh, Myanmar, Pakistan, Sri Lanka.
69. Hypolimnas misippus (Linnaeus, 1764);

Danaid Egg fly

Papilio misippus Linnaeus, 1764, Museum S'ae R'ae M'tis Ludovicae Ulricae Reginae Svecorum, Gothorum, Vandalorumque: 264. TL: America.

Specimen examined: $1 \hat{0}, \mathrm{MR}, 13 \cdot \mathrm{v} \cdot 2010, \mathrm{Nym} \cdot 53 \cdot \hat{0}, \mathrm{R}$. Lodh.

Conservation status: Tripura: rare; India: Schedule I (IWPA).

Distribution: India (common in almost all states of India, Tripura), Afghanistan, America, Bangladesh, Bhutan, Myanmar, Nepal, Sri Lanka, Pakistan.

70. Junonia almana (Linnaeus, 1758); Peacock Pansy Papilio almana Linnaeus, 1758, Systema naturae per regna tria naturae, secundum classes, ordines, genera, species, cum characteribus, differentiis, synonymis, locis (Ed. 10) 1: 472. TL: India; China.

Specimen examined: 10, PP, 3.vi.2009, Nym.57. $0^{\lambda}, \mathrm{R}$. Lodh; 1ㅇ, PP, 3.vi.2009, Nym.57. +, R. Lodh.

Conservation status: Tripura: very common; IUCN/ CITES: not evaluated.

Distribution: India (common in almost all states of India), Bangladesh, Bhutan, China, Myanmar, Nepal, Pakistan, Sri Lanka.

71. Junonia atlites (Linnaeus, 1763); Grey Pansy Papilio atlites Linnaeus, 1763, Centuria Insectorum Rariorum 6: 407. TL: Asia.

Specimen examined: $1 \hat{\delta} / q$ (monomorphic), MR, 13.v.2010, Nym.56.img. ${ }^{\lambda} / q$, R. Lodh.

Conservation status: Tripura: very common; IUCN/ CITES: not evaluated.

Distribution: India (common except in drier areas, Tripura), Bangladesh, Bhutan, Myanmar, Nepal, Sri Lanka.

\section{Junonia hierta (Fabricius, 1798); Yellow Pansy}

Papilio hierta Fabricius, 1798, Supplementum Entomologiae Systematicae: 424. TL: South India.

Specimen examined: $10^{\lambda}, \mathrm{KL}$, 9.x.2009, Nym.54. $\overbrace{}^{\lambda}, \mathrm{R}$. Lodh; 1ㅇ, KL, 9.x.2009, Nym.54. ㅇ, R. Lodh.

Conservation status: Tripura: common; IUCN/CITES: not evaluated.

Distribution: India (common in almost all states of India, Tripura), Afghanistan, Bangladesh, Bhutan, Myanmar, Nepal, Pakistan, Sri Lanka.

73. ${ }^{*}$ Junonia iphita (Cramer, 1782); Chocolate Pansy Papilio iphita Cramer, 1782, Uitlandsche Kapellen (Papillons exotiques) 3 (17-21): 30. TL: China.

Specimen examined: $1 \hat{\jmath}$, SWS, 14.i.2010, Nym.55. $\overbrace{}^{\lambda}$, R. Lodh; 1오, SWS, 14.i.2010, Nym.55. ㅇ, R. Lodh.

Conservation status: Tripura: occasional; IUCN/ CITES: not evaluated.

Distribution: India (common except in drier areas, Tripura), Bangladesh, Bhutan, China, Myanmar, Nepal, Pakistan, Sri Lanka. 
74. Junonia lemonias (Linnaeus, 1758); Lemon Pansy Papilio lemonias Linnaeus 1758, Systema naturae per regna tria naturae, secundum classes, ordines, genera, species, cum characteribus, differentiis, synonymis, locis (Ed. 10) 1: 473. TL: Canton, China.

Specimen examined: 10ึ, BM, 15.x.2009, Nym.58. $\overbrace{}^{\lambda}$, R. Lodh; 1ㅇ, BM, 15.x.2009, Nym.58. ․, R. Lodh.

Conservation status: Tripura: common; IUCN/CITES: not evaluated.

Distribution: India (common in almost all states of India, Tripura), Bangladesh, Bhutan, China, Myanmar, Nepal, Pakistan, Sri Lanka.

\section{Tribe Kallimini}

75. *Kallima inachus Boisduval, 1836; Orange Oak Leaf Kallima inachus Boisduval, 1836, in Cuvier, Le Règne Animal 2: 139. TL: West Himalaya.

Specimen examined: $1 \widehat{\delta} / \phi$ (monomorphic), DB, 29. vii.2009, Nym.59.img. $\widehat{\jmath} / q$, R. Lodh.

Conservation status: Tripura: very rare; IUCN/CITES: not evaluated.

Distribution: India [Eastern Ghats (up to south of Godavari River), Jammu and Kashmir in the west to Arunachal Pradesh in the east in Himalaya, Jharkhand, Madhya Pradesh, Maharashtra, Northeast, Tripura, West Bengal (Siliguri-Darjeeling range)], Bangladesh Bhutan, Myanmar, Nepal.

Tribe Nymphalini

\section{6. *Symbrenthia hippoclus (Cramer, 1782);}

\section{Common Jester}

Papilio hippoclus Cramer, 1782, Uitlandsche Kapellen (Papillons exotiques) 3 (17-21): 46. TL: Amboina, Indonesia.

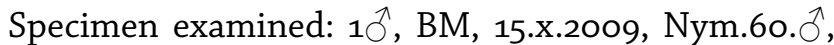
R. Lodh.

Conservation status: Tripura: rare; IUCN/CITES: not evaluated.

Distribution: India (Eastern Ghats, Himachal Pradesh in the west to Arunachal Pradesh in the east in Himalaya, Northeast, Tripura), Bangladesh, Bhutan, Myanmar, Nepal.

Subfamily Satyrinae

Tribe Elymniini

\section{Elymnias hypermnestra (Linnaeus, 1763);}

\section{Common Palm Fly}

Papilio hypermnestra Linnaeus, 1763, Centuria Insectorum Rariorum 6: 407. TL: Java, Indonesia.

Specimen examined: $1 \hat{0}, \mathrm{GP}, 10 . v i .2009$, Nym.61. $\sigma^{\lambda}$ R. Lodh; 1 ㅇ, SS, 13.ii.2010, Nym.61.․․ R. Lodh.

Conservation status: Tripura: occasional; IUCN/ CITES: not evaluated.

Distribution: India (Gujarat, Madhya Pradesh, Northeast, Tripura, Uttaranchal in the west to Arunachal Pradesh in the east in Himalaya, South India, Tripura,
West Bengal), Bangladesh, Bhutan, Indonesia, Myanmar, Nepal, Sri Lanka.

\section{Elymnias malelas (Hewitson, 1863);}

Spotted Palm Fly

Melanitis malelas Hewitson, 1863, Illustrations of new species of exotic butterflies selected chiefly from the collections of W. Wilson Saunders and William C. Hewitson 4 (Melanitis): 70. TL: Bengal, India.

Specimen examined: 10̂, BP, 11.xi.2009, Nym.62. $\sigma^{\Uparrow}, \mathrm{R}$. Lodh; 1 \%, KL, 9.x.2009, Nym.62.9, R. Lodh.

Conservation status: Tripura: rare; IUCN/CITES: not evaluated.

Distribution: India (north-western part, Sikkim, Tripura).

\section{9. *Elymnias patna (Westwood, 1851);}

Blue-Striped Palm Fly

Melanitis patna Westwood, 1851, The genera of diurnal Lepidoptera, comprising their generic characters, a notice of their habitats and transformations, and a catalogue of the species of each genus (2): 405 . TL: East India.

Specimen examined: $1 \delta / q$ (monomorphic), GD, 11.x.2009, Nym.63.img. $\widehat{\top} / q$, R. Lodh.

Conservation status: Tripura: rare; IUCN/CITES: not evaluated.

Distribution: India (Tripura, Uttaranchal in the west to Arunachal Pradesh in the east in Himalaya), Bangladesh, Bhutan, Myanmar, Nepal.

\section{0. *Lethe chandica (Moore, 1857);}

Angled Red Forester

Debis chandica Moore, 1857, in Horsfield and Moore, A Catalogue of the lepidopterous insects in the museum of the Honourable East-India Company 1: 219. TL: Darjeeling, India.

Specimen examined: $1 \hat{\sigma}, \mathrm{MB}, 16 . x \mathrm{ii.2009}, \mathrm{Nym} .66 . \hat{\sigma}$, R. Lodh; 1 ㅇ, MB, 16.xii.2009, Nym.66. + , R. Lodh.

Conservation status: Tripura: rare; IUCN/CITES: not evaluated.

Distribution: India (Arunachal Pradesh, Northeast, Sikkim, Tripura), Bhutan, Myanmar, Nepal.

81. *Lethe confusa Aurivillius, 1897; Banded Tree Brown Lethe confusa Aurivillius, 1897, Bemerkungen zu den von. J. Chr. Fabricius aus danischen Sammlungen beschriebenen Lepidopteren 18(3/4): 142. TL: India.

Specimen examined: $1 \hat{\jmath}$, PP, 3.vi.2009, Nym.67. ${ }^{\lambda}, \mathrm{R}$. Lodh; 1 + , PP, 3.vi.2009, Nym.67. 9 , R. Lodh.

Conservation status: Tripura: very rare; IUCN/CITES: not evaluated.

Distribution: India (Jammu and Kashmir in the west to Arunachal Pradesh in the east in Himalaya, Northeast, Tripura), Bhutan, Nepal, Myanmar, Pakistan.

\section{Lethe europa (Fabricius, 1775);}

Bamboo Tree Brown (Figure 23)

Papilio europa Fabricius, 1775, Systema Entomologiae, sistens insectorum classes, ordines, genera, species, adiectis synonymis, locis, descriptionibus, observationibus: 500. TL: South India. 


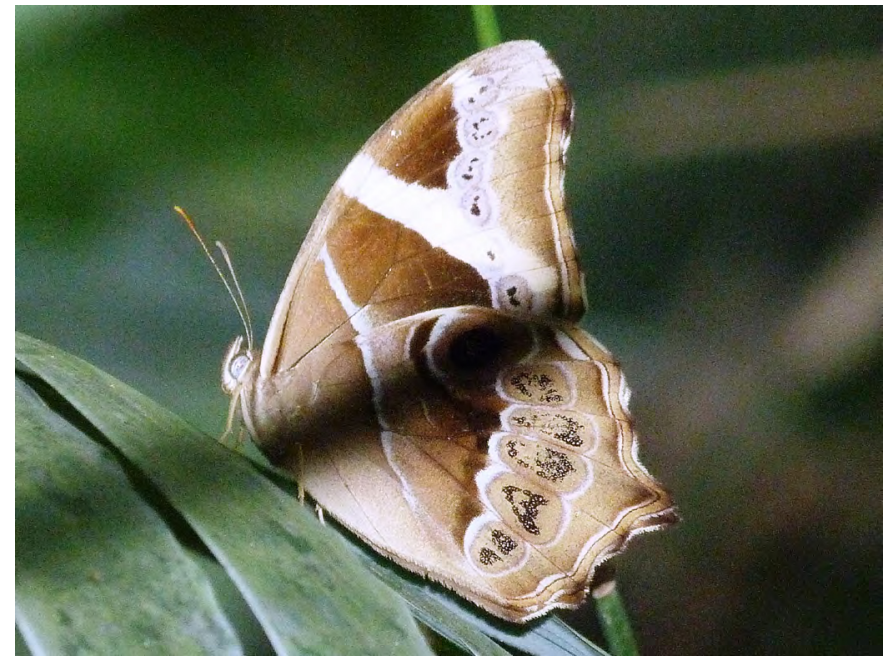

Figure 23. Lethe europa.

Specimen examined: 10 , RWS, 18.viii.2009, Nym.64. o, R. Lodh.

Conservation status: Tripura: rare; India: Schedule I (IWPA); IUCN: Critically Endangered.

Distribution: India (Jharkhand, Madhya Pradesh, Northeast, Orissa, South India, Tripura, Uttaranchal in the west to Arunachal Pradesh in the east in Himalaya, West Bengal), Bhutan, Nepal, Myanmar.

83. Lethe mekara (Moore, 1857); Common Red Forester Debis mekara Moore, 1857, A Catalogue of the lepidopterous insects in the museum of the Honourable East-India Company 1: 219. TL: Darjeeling, India.

Specimen examined: $1 \hat{\delta} / q$ (monomorphic), TWS, 18.v.2010, Nym.65.img. $\hat{\jmath} /$, R. Lodh.

Conservation status: Tripura: very rare; IUCN/CITES: not evaluated.

Distribution: India (Jharkhand, Madhya Pradesh, Northeast, Orissa, South India, Tripura, Uttaranchal in the west to Arunachal Pradesh in the east in Himalaya, West Bengal), Bhutan, Myanmar, Nepal.

\section{4. *Mycalesis anaxias Hewitson,1862;}

White Bar Bush Brown (Figure 24)

Mycalesis anaxias Hewitson, 1862, Illustrations of new species of exotic butterflies selected chiefly from the collections of W. Wilson Saunders and William C. Hewitson 4: 54. TL: Karnataka, South India.

Specimen examined: 1우, BC, 19.v.2011, Nym.70.우, R. Lodh.

Conservation status: Tripura: rare; India: Schedule II (IWPA).

Distribution: India (Arunachal Pradesh, Northeast, Sikkim, South India, Tripura), Bangladesh, Bhutan, Myanmar, Nepal.

85. *Mycalesis mineus (Linnaeus, 1758);

Dark-Brand Bush Brown

Papilio mineus Linnaeus, 1758, Systema naturae per regna tria naturae, secundum classes, ordines, genera, species, cum characteribus, differentiis, synonymis, locis (Ed. 10) 1: 471. TL: Canton, China.

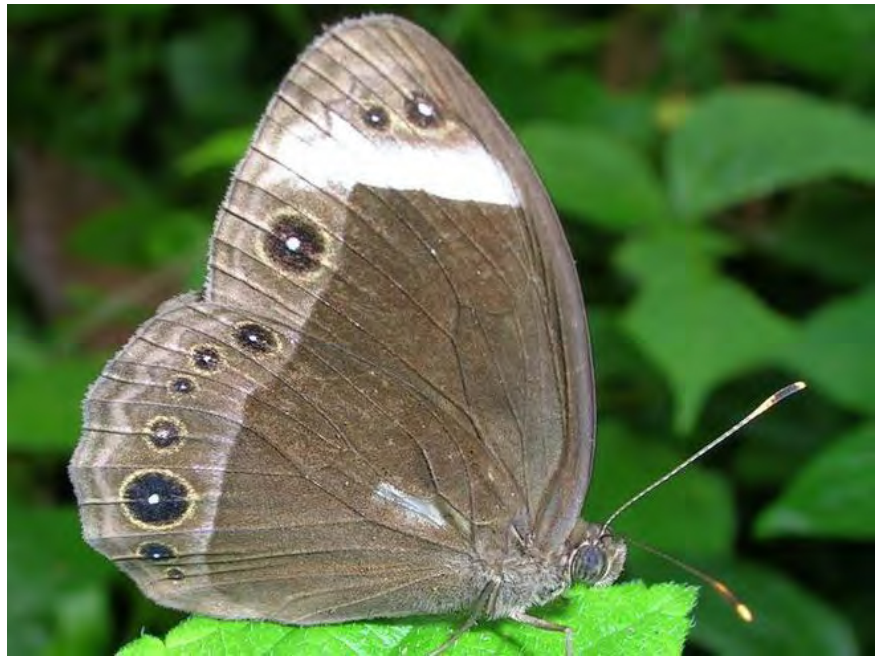

Figure 24. Mycalesis anaxias.

Specimen examined: $1 \hat{0}$, UK, 13.viii.2009, Nym.72. ${ }^{\lambda}$, R. Lodh; 1ㅇ, UK, 13.viii.2009, Nym.72. ㅇ, R. Lodh.

Conservation status: Tripura: rare; India: Schedule II (IWPA).

Distribution: India (Himachal Pradesh in the west to Arunachal Pradesh in the east in Himalaya, Madhya Pradesh, Maharashtra, Northeast, South India, Tripura, West Bengal), Bangladesh, Bhutan, China, Myanmar, Nepal, Sri Lanka.

\section{Mycalesis perseus (Fabricius, 1775);}

\section{Common Bush Brown}

Papilio perseus Fabricius, 1775, Systema Entomologiae, sistens insectorum classes, ordines, genera, species, adiectis synonymis, locis, descriptionibus, observationibus: 488. TL: Australia.

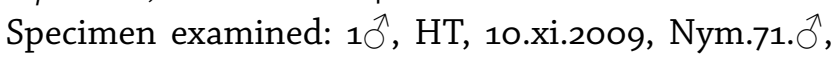
R. Lodh; 19, HT, 10.xi.2009, Nym.71. 9 , R. Lodh.

Conservation status: Tripura: occasional; IUCN/ CITES: not evaluated.

Distribution: India (Central and Eastern India, Himachal Pradesh in the west to Arunachal Pradesh in the east in Himalaya, Gujarat, Northeast, South India, Tripura), Australia, Bangladesh, Bhutan, Myanmar, Nepal, Pakistan, Sri Lanka.

87. ${ }^{*}$ Mycalesis suaveolens Wood-Mason \& de Nicéville, 1883; Wood-Mason's Bush Brown

Mycalesis suaveolens Wood-Mason and de Nicéville, 1883, The Butterflies of India, Burmah and Ceylon, a descriptive Handbook of all the known Species of rhopalocerous Lepidoptera inhabiting that Region, with Notices of allied Species occurring in the neighbouring Countries along the Border, with numerous Illustrations 1(2): 125. TL: Cachar, India.

Specimen examined: 10̂, CCRF, 20.viii.2009, Nym.74. ${ }^{\Uparrow}$, R. Lodh; 1우, CCRF, 20.viii.2009, Nym.74. 우, R. Lodh.

Conservation status: Tripura: rare; IUCN/CITES: not evaluated.

Distribution: India (Assam, Arunachal Pradesh, Sikkim, Tripura), Bangladesh, Bhutan, Myanmar, Nepal. 
88. ${ }^{*}$ Mycalesis visala Moore, 1857 ;

Long-brand Bush Brown

Mycalesis visala Moore, 1857, in Horsfield and Moore, A Catalogue of the lepidopterous insects in the museum of the Honourable East-India Company 1: 230. TL: Darjeeling, India.

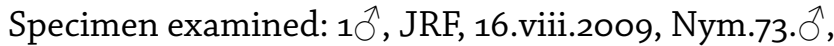
R. Lodh; 19, JRF, 16.viii.2009, Nym.73. ㅇ, R. Lodh.

Conservation status: Tripura: rare; IUCN: Critically Endangered.

Distribution: India [ (Himachal Pradesh in the west to Arunachal Pradesh in the east in Himalaya, Madhya Pradesh, Northeast, South India, Tripura, West Bengal), Bangladesh, Bhutan, Myanmar, Nepal, Sri Lanka.

Tribe Melanitini

89. Melanitis leda (Linnaeus, 1758);

\section{Common Evening Brown}

Papilio leda Linnaeus, 1758, Systema naturae per regna tria naturae, secundum classes, ordines, genera, species, cum characteribus, differentiis, synonymis, locis (Ed. 10) 1: 474. TL: Asia.

Specimen examined: $1{ }^{\lambda}$, SWS, 6.viii.2009, Nym.68. ${ }^{\lambda}$; 1오, SWS, 6.viii.2009, Nym.68. 9 , R. Lodh.

Conservation status: Tripura: very common; IUCN/ CITES: not evaluated.

Distribution: India (common almost all states of India except in extreme Northwest, Tripura), Bangladesh, Bhutan, Myanmar, Nepal, Pakistan, Sri Lanka.

\section{0. * Melanitis zitenius (Herbst, 1796);}

\section{Great Evening Brown}

Papilio zitenius Herbst, 1796, Natursystem aller bekannten in- und auslandischen Insekten als eine Fortsetzun der von Büffonschen Naturgeschichte 8: 5. TL: East coast north of Coromandel, India.

Specimen examined: $1 \hat{\delta} / \phi$ (monomorphic), SWS, 6.viii.2009, Nym.69.img. ${ }^{\lambda} /$ \% , R. Lodh.

Conservation status: Tripura: very rare; India: Schedule II (IWPA).

Distribution: India (Northeast, Tripura, Uttaranchal in the west to Arunachal Pradesh in the east in Himalaya, Western Ghats), Bhutan, Myanmar, Nepal.

91. Orsotrioena medus (Fabricius, 1775);

Smooth-eyed Bush Brown

Papilio medus Fabricius, 1775, Systema Entomologiae, sistens insectorum classes, ordines, genera, species, adiectis synonymis, locis, descriptionibus, observationibus: 488. TL: Java, Indonesia.

Specimen examined: 10 $0^{\lambda}$ PP, 3.vi.2009, Nym.75. $0^{\lambda}, \mathrm{R}$. Lodh; 1우 PP, 3.vi.2009, Nym.75.․, R. Lodh.

Conservation status: Tripura: very common; IUCN/ CITES: not evaluated.

Distribution: India (Madhya Pradesh, Northeast, Sikkim to Arunachal Pradesh in the east in Himalaya, South India up to Goa, Tripura), Bangladesh, Indonesia, Myanmar, Sri Lanka.

Tribe Satyrini
92. ${ }^{*} Y$ pthima asterope (Klug, 1832); Common Three Ring Hipparchia asterope Klug, 1832, in Ehrenberg, Symbolae Physicae 3: 29. TL: Africa.

Specimen examined: 10 , BC, 19.v.2011, Nym.78. ${ }^{\lambda}, \mathrm{R}$. Lodh.

Conservation status: Tripura: rare; IUCN/CITES: not evaluated.

Distribution: India (common in almost all states of India, Tripura), Afghanistan, Bangladesh, Bhutan, Myanmar, Nepal, Pakistan.

93. Ypthima baldus (Fabricius, 1775); Common Five Ring Papilio baldus Fabricius, 1775, Systema Entomologiae, sistens insectorum classes, ordines, genera, species, adiectis synonymis, locis, descriptionibus, observationibus: 829. TL: India.

Specimen examined: 10 , PP, 3.vi.2009, Nym.76. $\hat{0}$, R. Lodh; 19, PP, 3.vi.2009, Nym.76. +, R. Lodh.

Conservation status: Tripura: very common; IUCN/ CITES: not evaluated.

Distribution: India (Gujarat, Himachal Pradesh in the west to Arunachal Pradesh in the east in Himalaya, Madhya Pradesh, Northeast, South India, Tripura), Bangladesh, Bhutan, Myanmar, Nepal.

94. Ypthima huebneri Kirby, 1871; Common Four Ring Ypthima hübneri Kirby, 1871, A synonymic catalogue of the diurnal Lepidoptera: 95. TL: India.

Specimen examined: 10, HT, 10.xi.2009, Nym.77. $\overbrace{}^{\lambda}$, R. Lodh.

Conservation status: Tripura: common; IUCN/CITES: not evaluated.

Distribution: India (common in almost all states of India, Tripura), Bangladesh, Bhutan, Myanmar, Nepal, Sri Lanka.

95. *Ypthima nareda (Kollar, 1844); Large Three Ring

Satyrus nareda Kollar, 1844, in Hügel, Kaschmir und das Reich der Siek 4: 451. TL: India, Pakistan.

Specimen examined: 10, BC, 19.v.2011, Nym.79. $\hat{O}^{\lambda}$ R. Lodh; 1ㅇ, BC, 19.v.2011, Nym.79. ㅇ, R. Lodh.

Conservation status: Tripura: rare; IUCN/CITES: not evaluated.

Distribution: India (Gujarat, Himachal Pradesh in the west to Arunachal Pradesh in the east in Himalaya, Madhya Pradesh, Northeast, South India, Tripura), Bhutan, Bangladesh, Myanmar, Nepal.

Family Pieridae

Subfamily Coliadinae

96. Catopsilia pomona (Fabricius, 1775); Common Emigrant Papilio pomona Fabricius, 1775, Systema Entomologiae, sistens insectorum classes, ordines, genera, species, adiectis synonymis, locis, descriptionibus, observationibus: 479. TL: Holland.

Specimen examined: 10, BM, 15.x.2009, Pie.1. $\partial^{\lambda}, \mathrm{R}$. Lodh; 1오, BM, 15.x.2009, Pie.1.우, R. Lodh.

Conservation status: Tripura: very common; IUCN/ 
CITES: not evaluated.

Distribution: India (common in almost all states of India, Tripura), Afghanistan, Bangladesh, Bhutan, Holland (now the Netherlands), Myanmar, Nepal, Pakistan, Sri Lanka.

\section{Catopsilia pyranthe (Linnaeus, 1758);}

\section{Mottled Emigrant}

Papilio pyranthe Linnaeus, 1758, Systema naturae per regna tria naturae, secundum classes, ordines, genera, species, cum characteribus, differentiis, synonymis, locis (Ed. 10) 1: 469. TL: Canton, China.

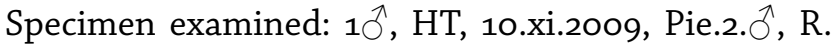
Lodh; 1ㅇ, HT, 10.xi.2009, Pie.2. 9 , R. Lodh.

Conservation status: Tripura: very common; IUCN/ CITES: not evaluated.

Distribution: India (common in almost all states of India, Tripura), Bangladesh, Bhutan, China, Myanmar, Nepal, Pakistan, Sri Lanka.

\section{8. *Eurema andersoni (Moore, 1886);}

One-spot Yellow Grass (Figure 25)

Terias andersoni Moore, 1886, The Journal of the Linnean Society of London 21(1): 47. TL: Mergui, South Myanmar.

Specimen examined: $1 \delta / q$ (monomorphic), AMNC, 17.i.2010, Pie.3.img. ${ }^{\hat{N}} / 9$, R. Lodh.

Conservation status: Tripura: occasional; India: Schedule II (IWPA); IUCN: Endangered.

Distribution: India (Andaman, Arunachal Pradesh, Northeast, Sikkim, South India, Tripura), Bangladesh, Bhutan, Myanmar, Sri Lanka.

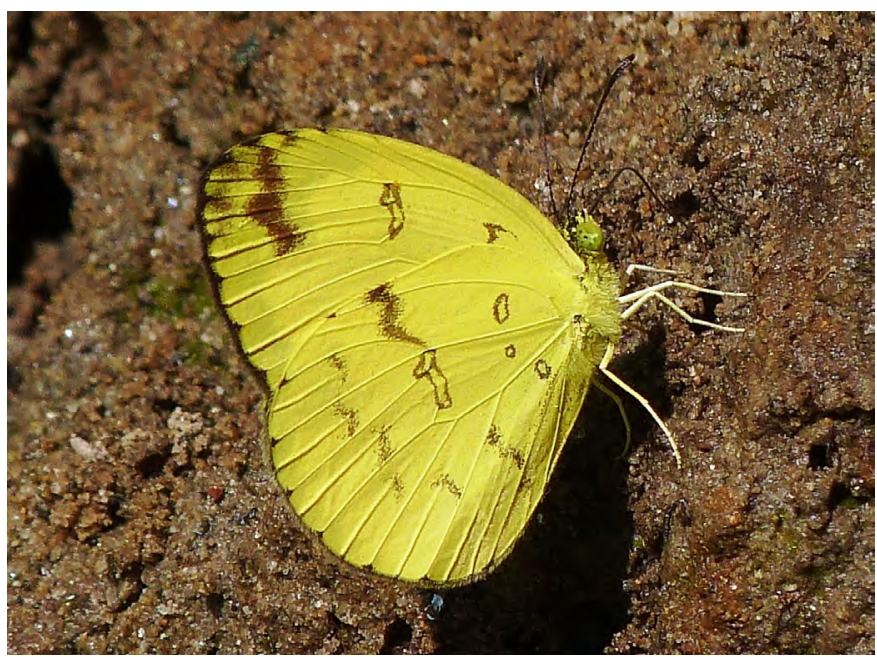

Figure 25. Eurema andersoni.

99. Eurema blanda (Boisduval, 1836);

Three-spot Grass Yellow

Terias blanda Boisduval, 1836, Histoire naturelle des insectes. Species général des lépidoptéres 1: 672. TL: Java, Indonesia.

Specimen examined: $1 \delta / \phi$ (monomorphic), GWS, 12.xi.2009, Pie.4.img. $\widehat{\jmath} /$ Q, R. Lodh.

Conservation status: Tripura: common; IUCN/CITES: not evaluated.
Distribution: India (Arunachal Pradesh, Sikkim, South India, Tripura), Bangladesh, Indonesia, Nepal.

100. Eurema hecabe (Linnaeus, 1758);

\section{Common Grass Yellow}

Papilio hecabe Linnaeus, 1758, Systema naturae per regna tria naturae, secundum classes, ordines, genera, species, cum characteribus, differentiis, synonymis, locis (Ed. 10) 1: 470. TL: South China; Hong Kong, China.

Specimen examined: $1 \hat{\partial}, \mathrm{MB}, 16 . x \mathrm{xi} .2009$, Pie.5. $\hat{0}, \mathrm{R}$. Lodh; 1ㅇ, MB, 16.xii.2009, Pie.5., R. Lodh.

Conservation status: Tripura: very common; IUCN/ CITES: not evaluated.

Distribution: India (common in almost all states of India, Tripura), Bangladesh, Bhutan, Myanmar, Nepal, Pakistan, Sri Lanka.

\section{Gandaca harina (Horsfield, 1829); Tree Yellow}

Terias harina Horsfield, 1829, Descriptive catalogue of the lepidopterous insects contained in the museum of the Honourable East-India Company (2): 137. TL: Java, Indonesia.

Specimen examined: $1 \hat{\delta} / \phi$ (monomorphic), DB, 14.v.2010, Pie.6.img. ${ }^{\lambda} / q$, R. Lodh.

Conservation status: Tripura: rare; IUCN/CITES: not evaluated.

Distribution: India (Arunachal Pradesh, Sikkim, Tripura), Bhutan, Indonesia, Myanmar, Nepal.

\section{Subfamily Pierinae}

Tribe Pierini

\section{2. *Appias libythea (Fabricius, 1775); Striped}

\section{Albatross (Figure 26)}

Papilio libythea Fabricius, 1775, Systema Entomologiae, sistens insectorum classes, ordines, genera, species, adiectis synonymis, locis, descriptionibus, observationibus: 471. TL: Asia.

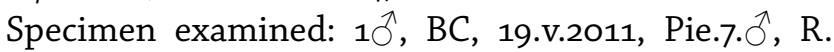
Lodh; 1ㅇ, BC, 19.v.2011, Pie.7. 9 , R. Lodh.

Conservation status: Tripura: rare; India: Schedule IV (IWPA); IUCN: Near Threatened.

Distribution: India (Gujarat, Arunachal Pradesh,

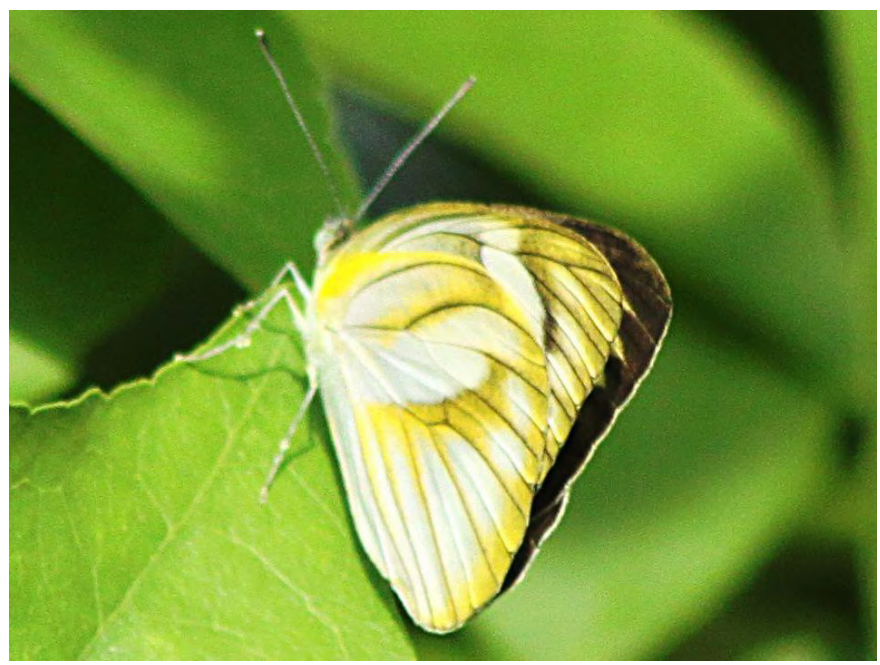

Figure 26. Appias libythea. 
Northeast, South India, Tripura, Uttar Pradesh, West Bengal), Bangladesh, Bhutan, Myanmar. Nepal, Pakistan, Sri Lanka.

103. *Appias lyncida (Cramer, 1777); Chocolate Albatross (Figure 27)

Papilio lyncida Cramer, 1777, Uitlandsche Kapellen (Papillons exotiques) 2 (9-16): p.: 52. TL: Java, Indonesia.

Specimen examined: $1 \hat{\jmath}$, TWS, 18.v.2010, Pie.8. $\partial^{\lambda}, \mathrm{R}$. Lodh; 1우, TWS, 18.v.2010, Pie.8. ㅇ, R. Lodh.

Conservation status: Tripura: occasional; IUCN: Near Threatened.

Distribution: India (Arunachal Pradesh, Nicobar Islands, Orissa, Sikkim, South India, Tripura, Uttaranchal), Bangladesh, Bhutan, Indonesia, Myanmar. Sri Lanka.

\section{4. ${ }^{*}$ Cepora nadina (Lucas, 1852); Lesser Gull}

(Figure 28)

Pieris nadina Lucas, 1852, Revue et Magasin de Zoologie Pure et Appliquée (2)4(7): 333. TL: Khasi Hills, India.

Specimen examined: $1 \hat{\delta} / \phi$ (monomorphic), DB, 14.v.2010, Pie.11.img. $\hat{O}^{\lambda} /$,, R. Lodh.

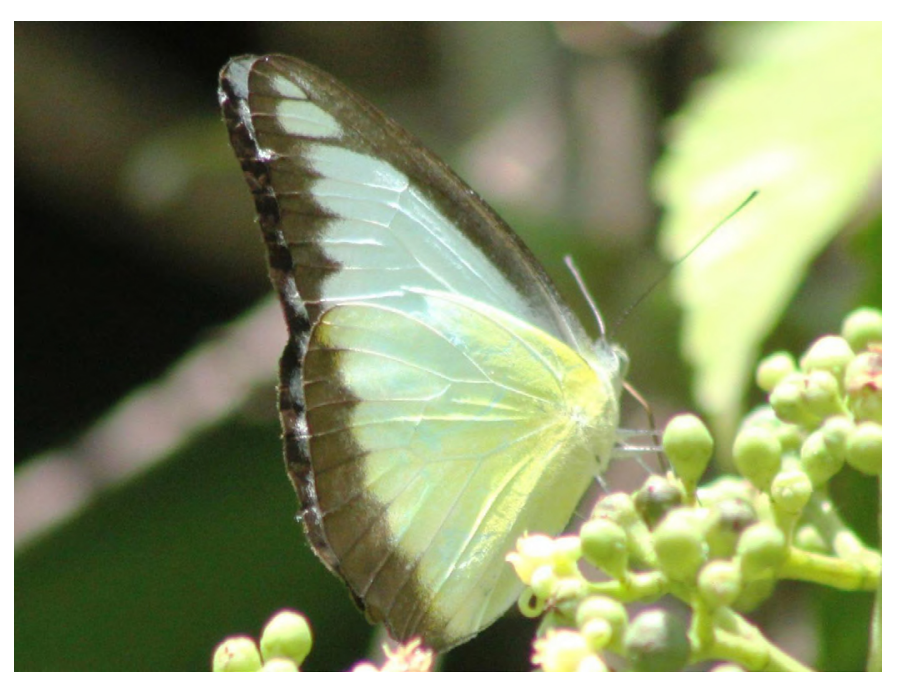

Figure 27. Appias lyncida.

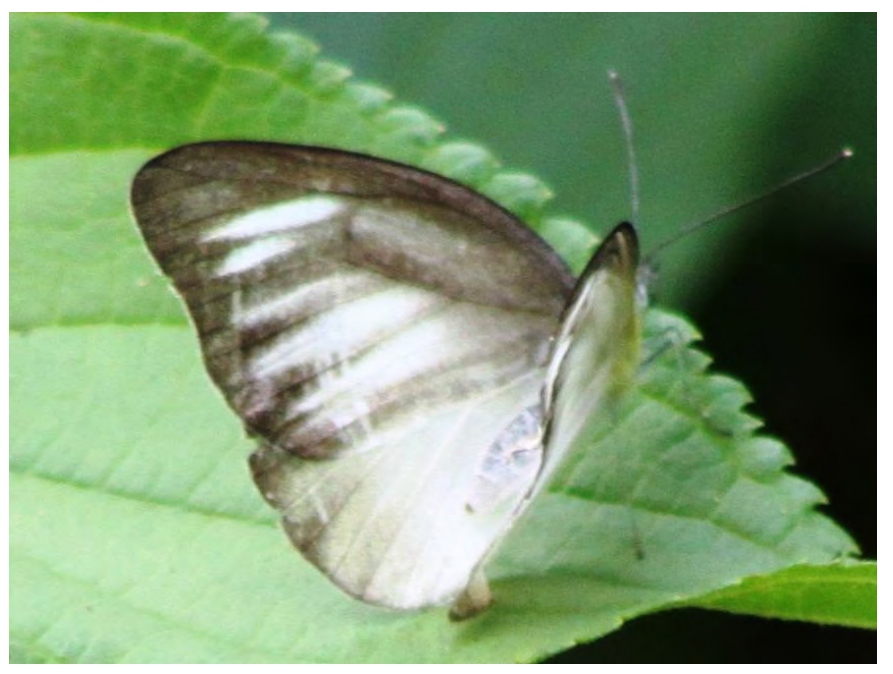

Figure 28. Cepora nadina.
Conservation status: Tripura: rare; India: Schedule II (IWPA), IUCN: Critically Endangered.

Distribution: India [Arunachal Pradesh, Assam, Sikkim, Tripura, Western Ghats (Maharashtra southwards to Kerala)], Bangladesh, Bhutan, Myanmar, Nepal, Sri Lanka.

105. ${ }^{*}$ Delias agostina (Hewitson, 1852); Yellow Jezebel Pieris agostina Hewitson, 1852, Illustrations of new species of exotic butterflies selected chiefly from the collections of W. Wilson Saunders and William C. Hewitson 1: 29. TL: Sikkim, India.

Specimen examined: $1 \hat{0}, \mathrm{BM}, 15 . x .2009$, Pie.14. ${ }^{\lambda}, \mathrm{R}$. Lodh; 1오, BM, 15.X.2009, Pie.14.ㅇ, R. Lodh.

Conservation status: Tripura: occasional; IUCN/ CITES: not evaluated.

Distribution: India (hills of northeast from Sikkim to Arunachal Pradesh, Tripura), Bhutan, Myanmar, Nepal.

106. ${ }^{*}$ Delias descombesi (Boisduval, 1836);

Red-spot Jezebel

Pieris descombesi Boisduval, 1836, Histoire naturelle des insectes. Species général des lépidoptéres 1: 465. TL: Cochin, China.

Specimen examined: 10 , TWS, 7.xi.2009, Pie.13. $\hat{0}, \mathrm{R}$. Lodh; 1우, TWS, 7.xi.2009, Pie.13. 우, R. Lodh.

Conservation status: Tripura: common; IUCN/CITES: not evaluated.

Distribution: India (hills of northeast from Sikkim to Arunachal Pradesh, Tripura), Bangladesh, Bhutan, China, Myanmar, Nepal.

107. Delias eucharis (Drury, 1773); Common Jezebel Papilio eucharis Drury, 1773, Illustrations of new species of exotic butterflies selected chiefly from the collections of W. Wilson Saunders and William C. Hewitson 2: 17. TL: India

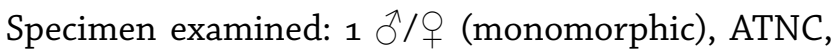
20.ix.2009, Pie.16.img. $\hat{\jmath} /$,, R. Lodh.

Conservation status: Tripura: rare; IUCN/CITES: not evaluated.

Distribution: India (common in almost all states of India except in arid region, Tripura), Bhutan, Nepal, North Myanmar, Pakistan, Sri Lanka.

108. *Delias hyparete (Linnaeus, 1758); Painted Jezebel

Papilio hyparete Linnaeus, 1758, Systema naturae per regna tria naturae, secundum classes, ordines, genera, species, cum characteribus, differentiis, synonymis, locis (Ed. 10) 1: 469. TL: Java, Indonesia.

Specimen examined: 10, BC, 19.v.2011, Pie.15. $\partial^{\lambda}, \mathrm{R}$. Lodh; 19, BC, 19.v.2011, Pie.15. , R. Lodh.

Conservation status: Tripura: occasional; IUCN/ CITES: not evaluated.

Distribution: India (Andhra Pradesh, Maharashtra, Orissa, Tamil Nadu, Tripura, Uttaranchal in the west to Arunachal Pradesh in the east in Himalaya, West Bengal), Bangladesh Bhutan, Indonesia, Myanmar, Nepal.

109. *Delias pasithoe (Linnaeus, 1767);

Red-base Jezebel 
Papilio pasithoe Linnaeus 1767 , Systema naturae per regna tria naturae, secundum classes, ordines, genera, species, cum characteribus, differentiis, synonymis, locis (Ed. 12) 1(2): 775. TL: Yunnan, South China.

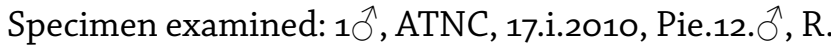
Lodh.

Conservation status: Tripura: occasional; IUCN/ CITES: not evaluated.

Distribution: India (hills of Northeast from Sikkim to Arunachal Pradesh, Tripura), Bangladesh, Bhutan, China, Myanmar, Nepal.

\section{0. *Hebomoia glaucippe (Linnaeus, 1758);}

\section{Great Orange Tip}

Papilio glaucippe Linnaeus, 1758, Systema naturae per regna tria naturae, secundum classes, ordines, genera, species, cum characteribus, differentiis, synonymis, locis (Ed. 10) 1: 469. TL: Canton, China.

Specimen examined: 10, BM, 15.x.2009, Pie.17. ${ }^{\lambda}, \mathrm{R}$. Lodh; 19, BM, 15.x.2009, Pie.17. ㅇ, R. Lodh.

Conservation status: Tripura: occasional; IUCN/ CITES: not evaluated.

Distribution: India (Andaman, hills of Northeast from Sikkim to Arunachal Pradesh, South India, Tripura), Bangladesh, Bhutan, China, Myanmar, Nepal, Sri Lanka.

111. *Ixias pyrene (Linnaeus, 1764): Yellow Orange Tip Papilio pyrene Linnaeus, 1764, Museum S'ae R'ae M'tis Ludovicae Ulricae Reginae Svecorum, Gothorum, Vandalorumque: 241. TL: Canton, China.

Specimen examined: $1 \hat{\jmath}$, BC, 19.v.2011, Pie.18.img. ${ }^{\Uparrow}$, R. Lodh.

Conservation status: Tripura: very rare; IUCN/CITES: not evaluated.

Distribution: India (common in almost all states of India, Tripura), Afghanistan, Bangladesh, Bhutan, Myanmar, Nepal, Pakistan, Sri Lanka.

112. Leptosia nina (Fabricius, 1793); Psyche

Papilio nina Fabricius, 1793, Entomologia systematica 3(1): 194. TL: India.

Specimen examined: $1 \sigma^{\lambda}, \mathrm{BC}, 18$. viii.2009, Pie.19. ${ }^{\lambda}, \mathrm{R}$.

Lodh; 19, BC, 18.viii.2009, Pie.19. ․, R. Lodh.

Conservation status: Tripura: common; IUCN/CITES: not evaluated.

Distribution: India (Andaman Islands, Tripura), Bangladesh, Nepal, Pakistan, Sri Lanka.

\section{Pareronia hippia (Fabricius, 1787);}

Common Wanderer

Papilio valeria Fabricius, 1787, Mantissa Insectorum (2): 55. TL: Asia.

Specimen examined: 1 ㅇ, BM, 15.x.2009, Pie.20. 9 , R. Lodh.

Conservation status: Tripura: rare; IUCN/CITES: not evaluated.

Distribution: India (common in almost all states of India, Tripura), Bangladesh, Bhutan, Myanmar, Nepal, Pakistan, Sri Lanka.
114. *Pieris brassicae (Linnaeus, 1758);

Large Cabbage White

Papilio brassicae Linnaeus, 1758, Systema naturae per regna tria naturae, secundum classes, ordines, genera, species, cum characteribus, differentiis, synonymis, locis (Ed. 10) 1: 467. TL: Sweden.

Specimen examined: 29, BC, 19.v. 2011, Pie.23.i. $q$, Pie.23.ii. , , R. Lodh.

Conservation status: Tripura: rare; IUCN/CITES: not evaluated.

Distribution: India (Andaman and Nicobar Islands, Tripura), Bangladesh, Bhutan, Myanmar, Nepal, Pakistan, Sri Lanka, Sweden.

115. Pieris canidia (Linnaeus, 1768);

Indian Cabbage White

Papilio canidia Linnaeus, 1768, Amoenitates Academicae 7(150): 504. TL: South China.

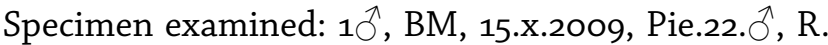
Lodh; 19, BM, 15.x.2009, Pie.22. 9 , R. Lodh.

Conservation status: Tripura: occasional; IUCN/ CITES: not evaluated.

Distribution: India (Jammu and Kashmir in the west to Arunachal Pradesh in the east in Himalaya, South India, Tripura), Afghanistan, Bangladesh, Bhutan, China, Myanmar, Nepal, Pakistan.

116. *Pieris napi (Linnaeus, 1758); Green-veined White Papilio napi Linnaeus, 1758, Systema naturae per regna tria naturae, secundum classes, ordines, genera, species, cum characteribus, differentiis, synonymis, locis (Ed. 10) 1: 468. TL: Sweden.

Specimen examined: $1 \hat{\jmath}$, BM, 15.x.2009, Pie.21. $\overbrace{}^{\Uparrow}, \mathrm{R}$. Lodh.

Conservation status: Tripura: very rare; IUCN/CITES: not evaluated.

Distribution: India (Jammu and Kashmir in the west to Arunachal Pradesh in the east in Himalaya, Tripura), Bhutan, Myanmar, Nepal, Pakistan, Sweden.

Tribe Colotini

117. * Colotis danae (Fabricius, 1775); Crimson Tip

Papilio danae Fabricius, 1775, Systema Entomologiae, sistens insectorum classes, ordines, genera, species, adiectis synonymis, locis, descriptionibus, observationibus: 476. TL: Asia.

Specimen examined: $1 \hat{\sigma}, \mathrm{KL}$, 2.iii.2010, Pie.9.img. $\sigma^{\lambda}$, R. Lodh.

Conservation status: Tripura: very rare; IUCN: Near Threatened.

Distribution: India (Gujarat, Madhya Pradesh, Punjab, South India, Tripura), Pakistan.

118. *Colotis vestalis (Butler, 1876); White Arab

Teracolus vestalis Butler, 1876, Proceedings of the Zoological Society of London (3): 135. TL: Asia

Specimen examined: 1 +, ATNC, 17.i.2010, Pie.10. img. 9 , R. Lodh

Conservation status: Tripura: very rare; IUCN/CITES: 
not evaluated.

Distribution: India (Gujarat, Madhya Pradesh, Punjab, Rajasthan, Tripura Uttar Pradesh), Pakistan.

Family Lycaenidae

Subfamily Lycaeninae

119. ${ }^{*}$ Heliophorus epicles (Godart, 1823); Purple Sapphire Polyommatus epicles Godart, 1823, Encyclopédie Méthodique 9(2): 646. TL: East Java, Indonesia.

Specimen examined: 1오, ATNC, 17.i.2010, Lyc.1.․, R. Lodh.

Conservation status: Tripura: occasional; IUCN/ CITES: not evaluated.

Distribution: India (Andaman, Northeast, Tripura, Uttaranchal in the west to Arunachal Pradesh in the east in Himalaya), Bangladesh, Bhutan, Indonesia, Myanmar, Nepal.

Subfamily Miletinae

Tribe Miletini

120. *Miletus chinensis C. Felder, 1862; Common Brownie Miletus chinensis C. Felder, 1862, Verhandlungen der ZoologischBotanischen Gesellschaft in Wien. Vienna 12(1/2): 488. TL: Hong Kong, China.

Specimen examined: 1우, TWS, 18.v.2010, Lyc.2.+, R. Lodh.

Conservation status: Tripura: rare; IUCN/CITES: not evaluated.

Distribution: India (Sikkim to Arunachal Pradesh in the east in Himalaya, Tripura), Bangladesh, Bhutan, China, Myanmar, Nepal.

Tribe Spalgini

121. Spalgis epius (Westwood, 1852); Ape Fly

Lucia epius Westwood, 1852, The genera of diurnal Lepidoptera, comprising their generic characters, a notice of their habitats and transformations, and a catalogue of the species of each genus (2): 502 . TL: North India.

Specimen examined: $1 \hat{0}$, TWS, 18.v.2010, Lyc.3. $\hat{O}^{\lambda}, \mathrm{R}$ Lodh; 19, TWS, 18.v.2010, Lyc.3.+, R. Lodh.

Conservation status: Tripura: rare; IUCN/CITES: not evaluated.

Distribution: India (Northeast, Tripura, Uttaranchal in the west to Arunachal Pradesh in the east in Himalaya, peninsular India up to Gujarat, West Bengal), Bangladesh, Bhutan, Myanmar, Nepal, Sri Lanka.

Subfamily Polyommatinae

Tribe Lycaenesthini

122. *Anthene emolus (Godart, 1823);

Common Ciliate Blue

Polyommatus emolus Godart, 1824, Encyclopédie Méthodique 9(2): 656. TL: Bengal, India.
Specimen examined: $1 \hat{\delta} / \phi$ (monomorphic), DB, 7.iv. 2010, Lyc.4.img. $\hat{O}^{\lambda} / Q$, R. Lodh.

Conservation status: Tripura: rare; IUCN/CITES: not evaluated.

Distribution: India [Northeast, Sikkim to Arunachal Pradesh in the east in Himalaya, South India up to Maharashtra and Orissa (Eastern Ghats), Tripura, West Bengal], Bangladesh, Bhutan, Myanmar, Nepal.

\section{3. *Anthene lycaenina (Felder, 1868);}

\section{Pointed Ciliate Blue}

Pseudodipsas lycaenina Felder, 1868, Verhandlungen der ZoologischBotanischen Gesellschaft in Wien. Vienna 18(1-2): 281. TL: Sri Lanka.

Specimen examined: $1 \delta / O$ (monomorphic), DB, 7.iv.2010, Lyc.5.img. ${ }^{\Uparrow} /$,, R. Lodh.

Conservation status: Tripura: very rare; India: Schedule II (IWPA); IUCN: vulnerable.

Distribution: India (Jharkhand, Northeast, Orissa, Sikkim in the west to Arunachal Pradesh in the east in Himalaya, South India up to Gujarat, Tripura, West Bengal), Bangladesh, Myanmar, Sri Lanka.

Tribe Polyommatini

124. * Caleta caleta (Hewitson, 1876); Angled Pierrot Lycaena caleta Hewitson 1876, Illustrations of new species of exotic butterflies selected chiefly from the collections of W. Wilson Saunders and William C. Hewitson 5: 69. TL: Celebes, Indonesia.

Specimen examined: $1 \hat{\delta} / P$ (monomorphic), DB, 7.iv.2010, Lyc.7.img. $\hat{O} /$,, R. Lodh.

Conservation status: Tripura: rare; IUCN/CITES: not evaluated.

Distribution: India (peninsular India up to Gujarat and Madhya Pradesh, Tripura, Uttaranchal in the west to Arunachal Pradesh in the east in Himalaya, West Bengal), Bhutan, Indonesia, Myanmar, Nepal, Sri Lanka.

125. *Caleta elna (Hewitson, 1876); Elbowed Pierrot Lycaena elna Hewitson 1876, Illustrations of new species of exotic butterflies selected chiefly from the collections of W. Wilson Saunders and William C. Hewitson (5) (Lycaena): 70, pl. 35, f. 8. TL: Java, Indonesia.

Specimen examined: $1 \hat{\jmath} / 9$ (monomorphic), DB, 7.iv.2010, Lyc.6.img. $\widehat{\jmath} /$, , R. Lodh.

Conservation status: Tripura: very rare; IUCN/CITES: not evaluated.

Distribution: India (Orissa, Sikkim in the west to Arunachal Pradesh in the east in Himalaya, Tripura), Bangladesh, Bhutan, Indonesia, Myanmar, Nepal.

\section{Castalius rosimon (Fabricius, 1775); Common}

Pierrot (Figure 29)

Papilio rosimon Fabricius, 1775, Systema Entomologiae, sistens insectorum classes, ordines, genera, species, adiectis synonymis, locis, descriptionibus, observationibus: 523. TL: Tranquebar, India.

Specimen examined: $1 \hat{0}$, BM, 15.x.2009, Lyc.8. $O^{\hat{N}}, \mathrm{R}$. Lodh; 1ㅇ, TWS, 18.v.2010, Lyc.8. + , R. Lodh.

Conservation status: Tripura: very common; India: 


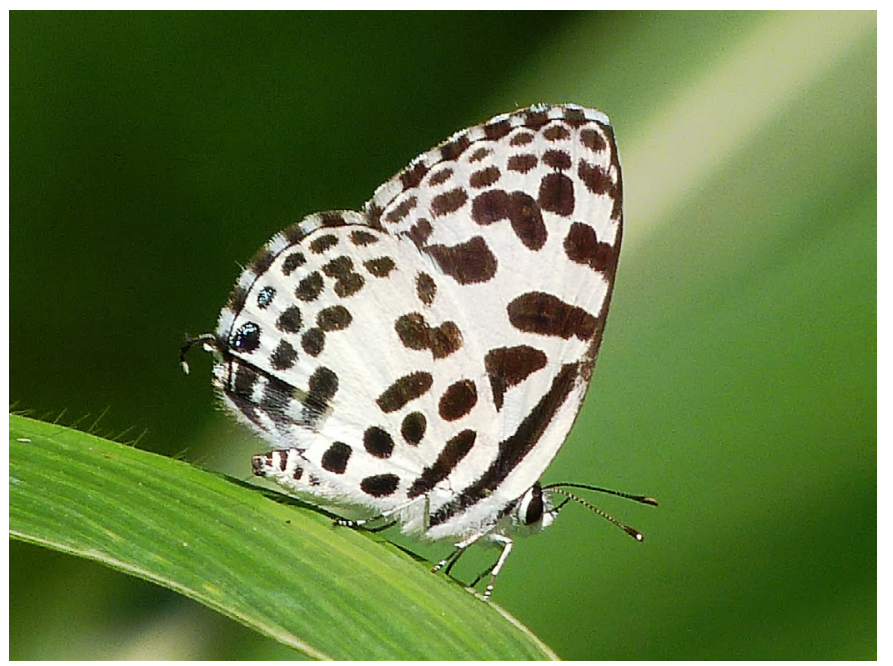

Figure 29. Castalius rosimon.

Schedule I (IWPA).

Distribution: India (common in almost all states of India except in Northwest, Tripura), Bangladesh, Bhutan, Myanmar, Nepal, Pakistan, Sri Lanka.

\section{7. * Catochrysops strabo (Fabricius, 1793);}

Forget-Me-Not

Hesperia strabo Fabricius, 1793, Entomologia systematica 3(1): 287. TL: India.

Specimen examined: $10^{\lambda}, \mathrm{DB}$, 14.v.2010, Lyc.9. $\overbrace{}^{\lambda}, \mathrm{R}$. Lodh; 1이 DB, 14.v.2010, Lyc.9. ㅇ, R. Lodh.

Conservation status: Tripura: very rare; IUCN/CITES: not evaluated.

Distribution: India (common in almost all states of India, Tripura), Bangladesh, Bhutan, Nepal, Pakistan, Sri Lanka.

\section{Chilades lajus (Cramer, 1782); Lime Blue}

Papilio laius Cramer, 1782, Uitlandsche Kapellen (Papillons exotiques) 4(26b-28): 62. TL: Coromandel Coast, India.

Specimen examined: $1 \hat{0}, \mathrm{BM}, 15 \cdot x \cdot 2009$, Lyc.11. $\overbrace{}^{\lambda}, \mathrm{R}$. Lodh; 19, BM, 15.x.2009, Lyc.11.+, R. Lodh.

Conservation status: Tripura: occasional; IUCN/ CITES: not evaluated.

Distribution: India (common in almost all states of India, Tripura), Bangladesh, Bhutan, Myanmar, Nepal, Pakistan, Sri Lanka.

129. ${ }^{*}$ Chilades pandava (Horsfield, 1829); Plains Cupid Lycaena pandava Horsfield, 1829, Descriptive catalogue of the lepidopterous insects contained in the museum of the Honourable EastIndia Company (2): 84. TL: Java, Indonesia.

Specimen examined: $1 \hat{\delta} / 9$ (monomorphic), DB, 14.v.2010, Lyc.10.img. $\widehat{\jmath} /$, R. Rodh.

Conservation status: Tripura: very rare; IUCN/CITES: not evaluated.

Distribution: India (Andaman and Nicobar Islands, Tripura), Bangladesh, Bhutan, Indonesia, Myanmar, Nepal, Pakistan, Sri Lanka.
130. Discolampa ethion (Westwood, 1851);

\section{Banded Blue Pierrot}

Lycaena ethion Westwood, 1851, The genera of diurnal Lepidoptera, comprising their generic characters, a notice of their habitats and transformations, and a catalogue of the species of each genus (2): 490, pl. 76. TL: Sri Lanka.

Specimen examined: $1 \hat{\delta} / q$ (monomorphic), SB, 16.x. 2009, Lyc.12.img. $\hat{o} /$,, R. Lodh.

Conservation status: Tripura: rare; IUCN/CITES: not evaluated.

Distribution: India [Andaman and Nicobar Islands, Assam to Arunachal Pradesh in the east in Himalaya, Sikkim, Tripura, Uttaranchal, Western Ghats (Gujarat southwards)], Bangladesh, Myanmar, Sri Lanka.

\section{1. *Euchrysops cnejus (Fabricius, 1798); Gram Blue}

\section{(Figure 30)}

Hesperia cnejus Fabricius, 1798, Entomologia systematica: 430. TL: Tranquebar, India.

Specimen examined: $1 \delta / q$ (monomorphic), MR, 13.v.2010, Lyc.13.img. $\hat{\jmath} / 9$, R. Lodh.

Conservation status: Tripura: rare; India: Schedule II (IWPA).

Distribution: India (common almost in all states of India, Tripura), Bangladesh, Bhutan, Myanmar, Nepal, Pakistan, Sri Lanka.

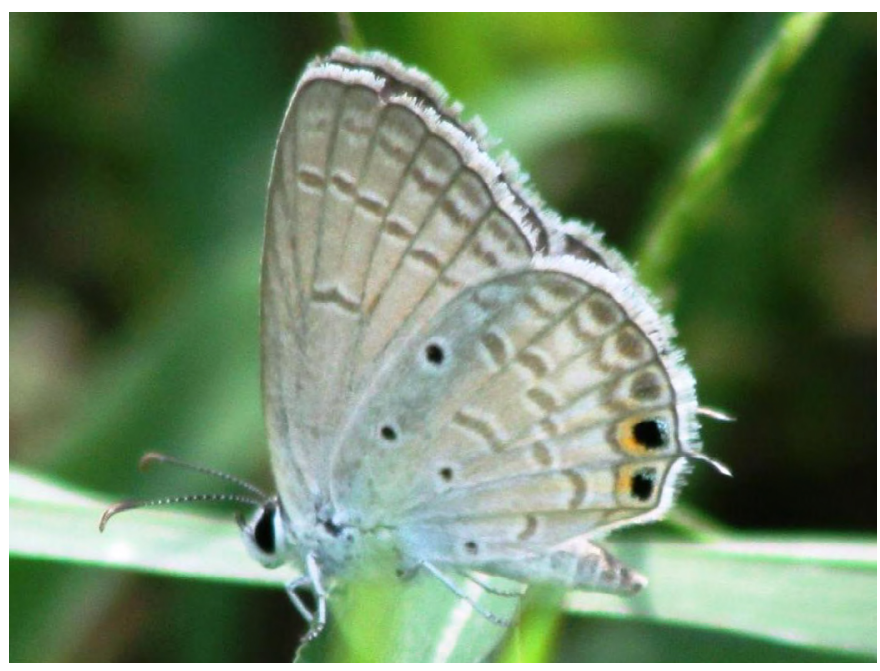

Figure 30. Euchrysops cnejus.

132. * Jamides alecto (Felder, 1860); Metalic Cerulean Lycaena alecto Felder, 1860, Sitzungsberichte der Kaiserlichen Akademie der Wissenschaften 40(11): 456.TL: Ambon, Indonesia.

Specimen examined: $1 \hat{\delta} / \phi$ (monomorphic), CB,

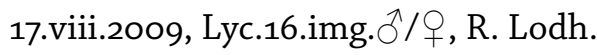

Conservation status: Tripura: occasional; India: Schedule II (IWPA), IUCN: Near Threatened.

Distribution: India (Sikkim in the west to Arunachal Pradesh in the east in Himalaya, Tripura, Western Ghats from Karnataka southwards), Nepal, Bangladesh, Bhutan, Indonesia, Myanmar, Sri Lanka. 
133. *Jamides bochus (Stoll, 1782); Dark Cerulean

Papilio bochus Stoll, 1782, Uitlandsche Kapellen (Papillons exotiques) 4(32-32): 210. TL: Coromandel Coast, South India.

Specimen examined: 1 ㅇ, BM, 15.x.2009, Lyc.14.ㅇ, R. Lodh.

Conservation status: Tripura: occasional; IUCN/ CITES: not evaluated.

Distribution: India (common in almost all states of India except in arid Northwest, Tripura), Bangladesh, Bhutan, Nepal, Pakistan.

134. *Jamides celeno (Cramer, 1775); Common Cerulean Papilio celeno Cramer, 1775, Uitlandsche Kapellen (Papillons exotiques) 1(1-7): 31. TL: India.

Specimen examined: $1 \hat{\jmath}$, GWS, 12.xi.2009, Lyc.15. $\overbrace{}^{\lambda}$, R. Lodh; 1ㅇ, TWS, 18.v.2010, Lyc.15.ㅇ, R. Lodh.

Conservation status: Tripura: occasional; IUCN/ CITES: not evaluated.

Distribution: India (common in almost all states of India except in arid Northwest, Tripura), Afghanistan, Bangladesh, Bhutan, Myanmar, Nepal, Pakistan, Sri Lanka.

135. *Lampides boeticus (Linnaeus, 1767); Pea Blue

(Figure 31)

Papilio boeticus Linnaeus, 1767 , Systema naturae per regna tria naturae, secundum classes, ordines, genera, species, cum characteribus, differentiis, synonymis, locis (Ed. 12) 1(2): 789. TL: Algeria.

Specimen examined: $1 \widehat{\jmath}$, GWS, 12.xi.2009, Lyc.18. $\precsim$, R. Lodh; 19 , GWS, 12.xi.2009, Lyc.18. 9 , R. Lodh.

Conservation status: Tripura: occasional; India: Schedule II (IWPA).

Distribution: India (common in almost all states of India, Tripura), Afghanistan, Algeria, Bangladesh, Bhutan, Myanmar, Nepal, Pakistan, Sri Lanka.

136. *Leptotes plinius (Fabricius, 1793); Zebra Blue Hesperia plinius Fabricius, 1793, Entomologia systematica 3(1): 284. TL: Asia.

Specimen examined: 1 ㅇ, TWS, 7.xi.2009, Lyc.17.우, R. Lodh.

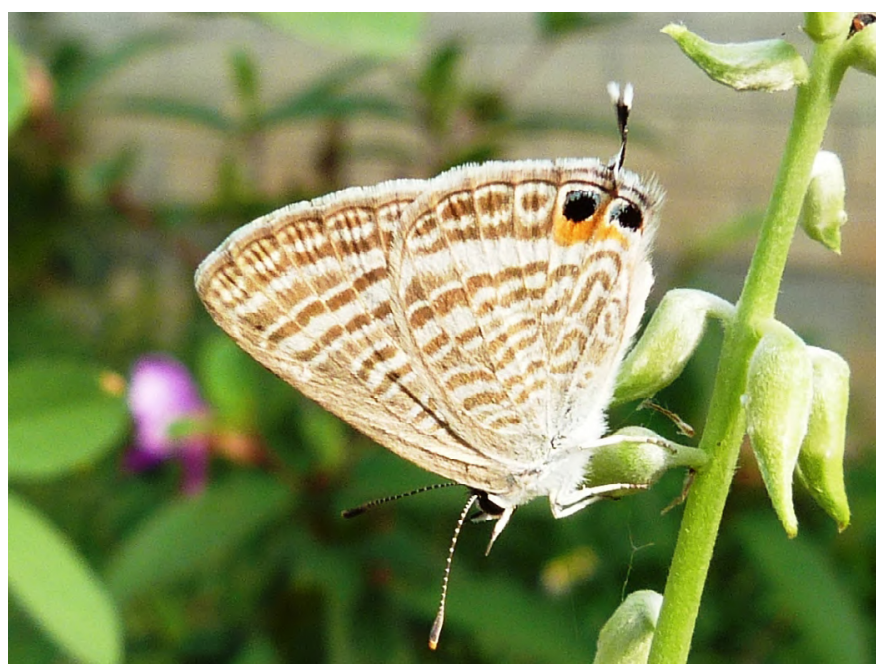

Figure 31. Lampides boeticus.
Conservation status: Tripura: very rare; IUCN/CITES: not evaluated.

Distribution: India (common in almost all states of India, Tripura), Bhutan, Myanmar, Nepal, Pakistan, Sri Lanka.

\section{Megisba malaya (Horsfield, 1828); Malayan}

\section{(Figure 32)}

Lycaena malaya Horsfield, 1828, Descriptive catalogue of the lepidopterous insects contained in the museum of the Honourable East-India Company (1): 70. TL: Java, Indonesia.

Specimen examined: $10 \hat{\sigma}$, ATNC, 8.iv.2012, Lyc.19. $\sigma^{\lambda}$ R. Lodh.

Conservation status: Tripura: rare; India: Schedule II (IWPA).

Distribution: India [Eastern Ghats, Northeast, Tripura, Uttaranchal in the west to Arunachal Pradesh in the east in Himalaya, Western Ghats (Maharashtra southwards) up to West Bengal], Bangladesh, Bhutan, Indonesia, Myanmar, Nepal, Sri Lanka.

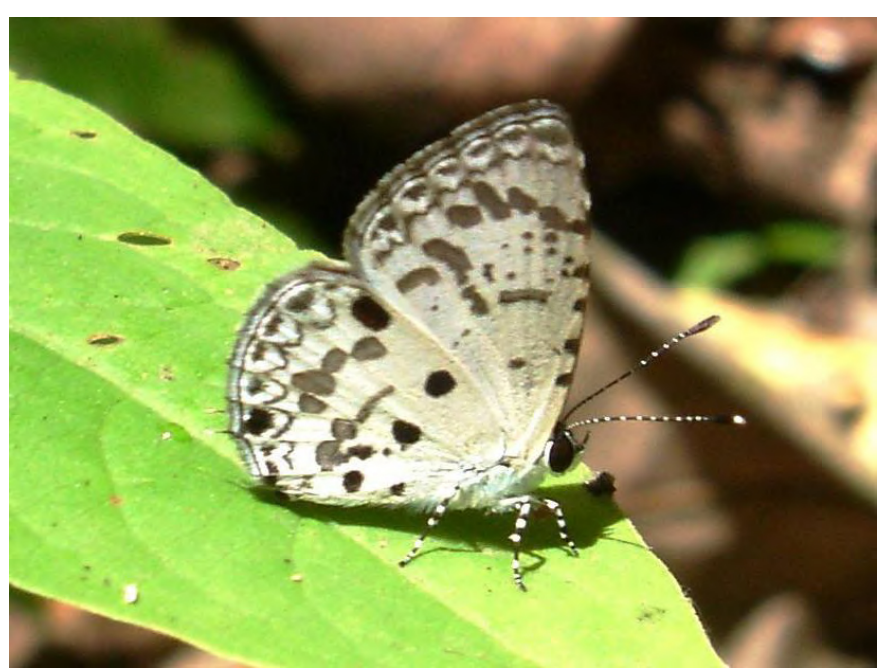

Figure 32. Megisba malaya.

138. Nacaduba kurava (Moore, 1858);

Transparent Six-line Blue

Lycaena kurava Moore 1858, in Horsfield \& Moore, A catalogue of the lepidopterous insects in the museum of the Honourable East-India Company 1: 22. TL: Java, Indonesia.

Specimen examined: 19, DB, 14.v.2010, Lyc.20.a. 9, R. Lodh.

Conservation status: Tripura: very rare; IUCN/CITES: not evaluated.

Distribution: India (Andaman and Nicobar Islands, Northeast, Sikkim in the west to Arunachal Pradesh in the east in Himalaya, South India up to Gujarat, Tripura), Bhutan, Indonesia, Bangladesh, Myanmar, Nepal, Sri Lanka.

139. *Neopithecops zalmora (Butler, 1870); Quaker Pithecops zalmora Butler, 1870, Catalogue of diurnal Lepidoptera described by Fabricius in the collection of the British Museum: 161. TL: Moulmein, Myanmar. 
Specimen examined: $1 \hat{\delta} / Q$ (monomorphic), KL, 2.iii.2010, Lyc.21.img. ${ }^{\lambda} / 9$, R. Lodh.

Conservation status: Tripura: rare; IUCN/CITES: not evaluated.

Distribution: India [Northeast, Tripura, Uttaranchal in the west to Arunachal Pradesh in the east in Himalaya, Western Ghats (Maharashtra southwards) and Eastern Ghats in peninsular India], Bangladesh, Bhutan, Myanmar, Nepal, Sri Lanka.

140. ${ }^{*}$ Prosotas bhutea (de Nicéville, 1883);

Bhutya Line Blue

Nacaduba bhutea de Nicéville, 1883, The Journal of the Asiatic Society of Bengal 52(2/4): 72. TL: Sikkim, India.

Specimen examined: $1 \hat{\delta} / 9$ (monomorphic), CB, 17.viii.2009, Lyc.23.img. $\widehat{\jmath} / 9$, R. Lodh.

Conservation status: Tripura: very rare; IUCN/CITES: not evaluated.

Distribution: India (Sikkim in the west to Arunachal Pradesh in the east in Himalaya, Tripura), Bhutan, Myanmar, Nepal.

141. ${ }^{*}$ Prosotas nora (Felder, 1860); Common Line Blue Lycaena nora Felder, 1860, Sitzungsberichte der Kaiserlichen Akademie der Wissenschaften 40(11): 458. TL: Amboina, Indonesia.

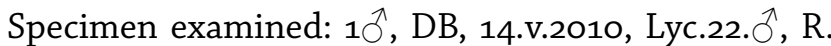
Lodh.

Conservation status: Tripura: rare; IUCN/CITES: not evaluated.

Distribution: India (common in almost all states of India, Tripura), Bangladesh, Bhutan, Indonesia, Myanmar, Nepal, Pakistan, Sri Lanka.

142. *Pseudozizeeria maha (Kollar, 1848); Pale Grass Blue Lycaena maha Kollar, 1848, in Hügel, Kaschmir und das Reich der Siek 4: 422. TL: Mussoorie, India.

Specimen examined: $1 \hat{\delta} / q$ (monomorphic), CB, 17.viii.2009, Lyc.24.img. $\sigma^{\lambda} /$, , R. Lodh.

Conservation status: Tripura: occasional; IUCN/ CITES: not evaluated.

Distribution: India (common in almost all states of India, Tripura), Bangladesh, Bhutan, Myanmar, Nepal, Pakistan, Sri Lanka.

143. *Tarucus extricates Butler, 1886; Rounded Pierrot Tarucus extricatus Butler, 1886, Proceedings of the Zoological Society of London (3): 366. TL: Asia.

Specimen examined: 10 , SWS, 14.i.2010, Lyc.25. $\partial^{\lambda}, \mathrm{R}$. Lodh.

Conservation status: Tripura: very rare; IUCN/CITES: not evaluated.

Distribution: India (Maharashtra, Punjab, Rajasthan, Saurahtra (now in Gujarat), Tripura); Afghanistan, Pakistan.

144. Zizeeria karsandra (Moore, 1865); Dark Grass Blue (Figure 33)

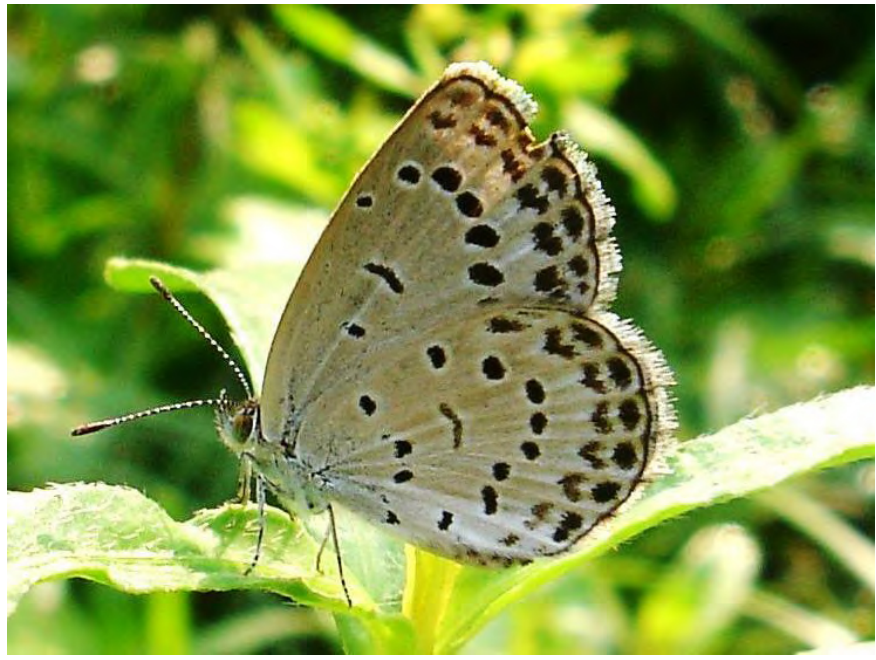

Figure 33. Zizeeria karsandra.

Polyommatus karsandra Moore, 1865, Proceedings of the Zoological Society of London (2): 505. TL: India.

Specimen examined: 1우, HT, 10.xi.2009, Lyc.26. 우, R. Lodh.

Conservation status: Tripura: occasional; IUCN: vulnerable.

Distribution: India (common in almost all states of India, Tripura), Afghanistan, Bangladesh, Bhutan, Myanmar, Nepal, Pakistan, Sri Lanka.

145. Zizina otis (Fabricius, 1787); Lesser Grass Blue Papilio otis Fabricius, 1787, Mantissa Insectorum 2: 73. TL: China.

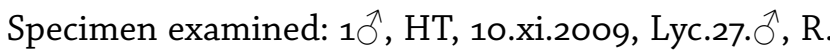
Lodh; 1ㅇ, HT, 10.xi.2009, Lyc.27. 9 , R. Lodh.

Conservation status: Tripura: very common; IUCN/ CITES: not evaluated.

Distribution: India (common in almost all states of India, Tripura), Bangladesh, Bhutan, China, Myanmar, Nepal, Pakistan, Sri Lanka.

146. Zizula hylax (Fabricius, 1775); Tiny Grass Blue Papilio hylax Fabricius, 1775, Systema Entomologiae, sistens insectorum classes, ordines, genera, species, adiectis synonymis, locis, descriptionibus, observationibus: 526. TL: Tranquebar, India.

Specimen examined: $1 \hat{\delta} / \phi$ (monomorphic), KL, 2.iii.2010, Lyc.28.img. $\overbrace{}^{\Uparrow} / q$, R. Lodh.

Conservation status: Tripura: occasional; IUCN/ CITES: not evaluated.

Distribution: India (common in almost all states of India, Tripura), Bangladesh, Bhutan, Myanmar, Nepal, Pakistan, Sri Lanka.

Subfamily Poritiinae

Tribe Poritiini

147. ${ }^{*}$ Poritia hewitsoni Moore, 1865 ; Common Gem (Figure 33)

Poritia hewitsoni Moore, 1865, Proceedings of the Zoological Society of London (2): 775. TL: Nepal. 


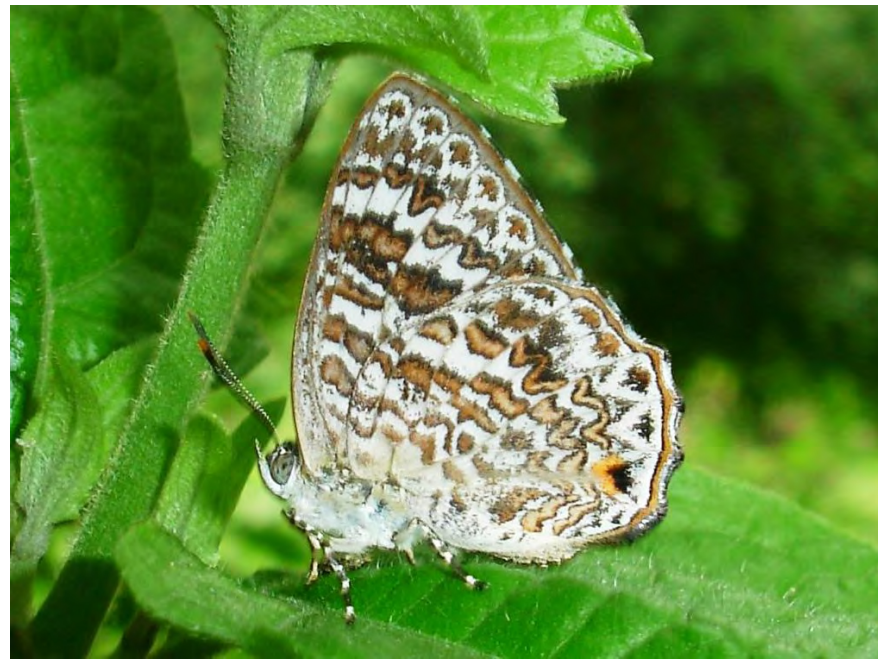

Figure 34. Poritia hewitsoni.

Specimen examined: 10, ATNC, 17.i.2010, Lyc.29. ${ }^{\lambda}$, R. Lodh; 19, ATNC, 17.i.2010, Lyc.29.+, R. Lodh.

Conservation status: Tripura: rare; India: Schedule II (IWPA).

Distribution: India (Uttaranchal in the west to Arunachal Pradesh in the east in Himalaya, Tripura), Bhutan, Bangladesh, Myanmar, Nepal.

Subfamily Theclinae

Tribe Amblypodiini

148. Iraota timoleon (Stoll, 1790); Silver Streak Blue (Figure 35)

Papilio timoleon Stoll, 1790, Aanhangsel van het werk, De uitlandsche kapellen (2-5): 146. TL: China.

Specimen examined: $10^{\Uparrow}$, TWS, 7.xi.2009, Lyc.40. $0^{\Uparrow}, \mathrm{R}$. Lodh.

Conservation status: Tripura: very rare; IUCN: Endangered.

Distribution: India (Northeast, peninsular India up to Gujarat, Tripura, Uttar Pradesh, Uttaranchal in the west to Arunachal Pradesh in the east in Himalaya,

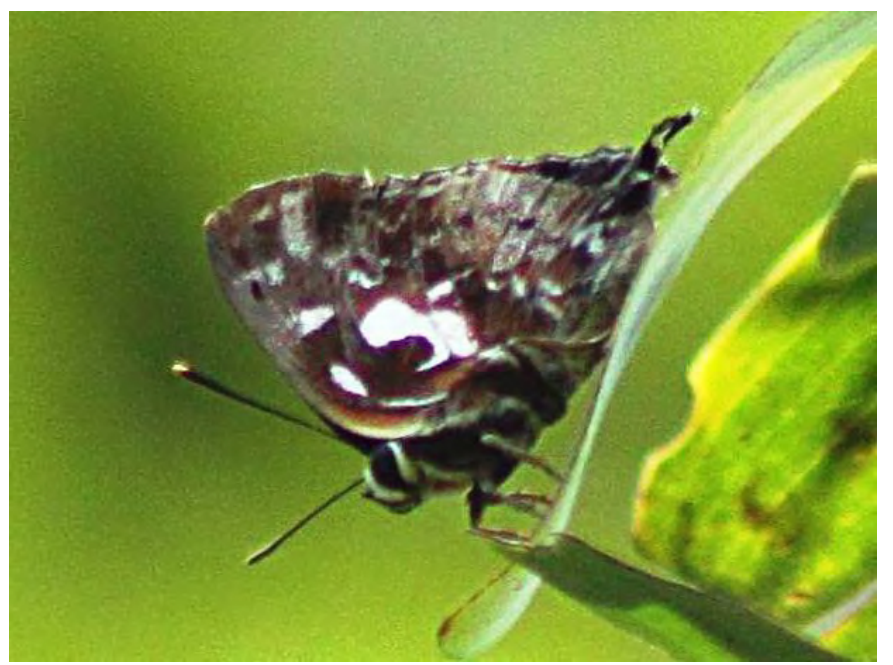

Figure 35. Iraota timoleon.

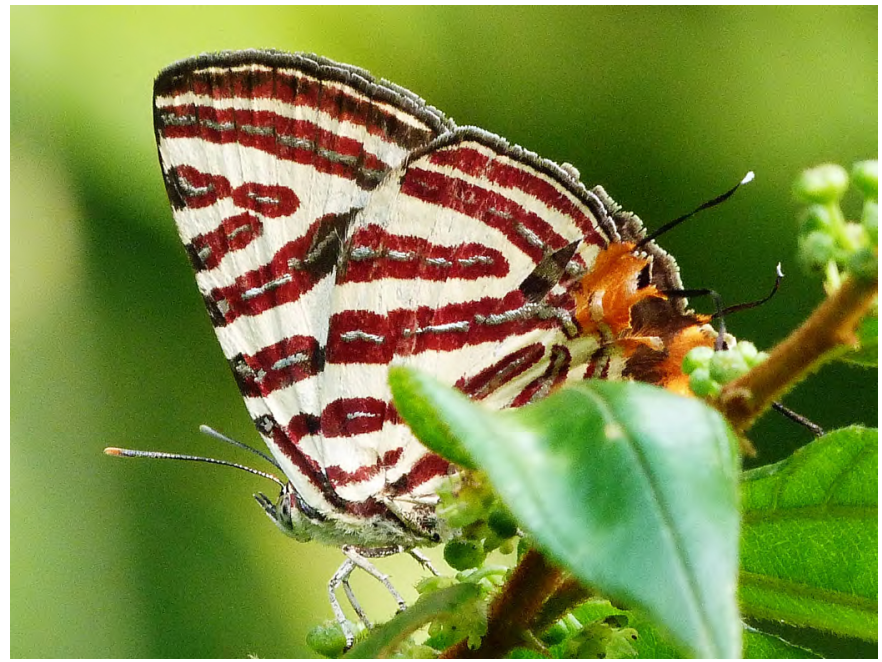

Figure 36. Spindasis lohita.

West Bengal), Bangladesh, Bhutan, Myanmar, Nepal, Sri Lanka.

Tribe Aphnaeini

149. *Spindasis lohita (Horsfield, 1829);

Long-banded Silverline (Figure 36)

Amblypodia lohita Horsfield, 1829, Descriptive catalogue of the lepidopterous insects contained in the museum of the Honourable EastIndia Company (2): 106. TL: Java, Indonesia.

Specimen examined: $10^{\lambda}, \mathrm{HT}$, 10.xi.2009, Lyc.47. $0^{\lambda}, \mathrm{R}$. Lodh.

Conservation status: Tripura: rare; India: Schedule II (IWPA), IUCN: Endangered.

Distribution: India (peninsular India up to Madhya Pradesh, Tripura, Uttaranchal in the west to Arunachal Pradesh in the east in Himalaya, West Bengal), Bangladesh, Bhutan, Indonesia, Myanmar, Nepal, Sri Lanka.

150. *Spindasis syama (Horsfield, 1829);

\section{Club Silverline}

Amblypodia syama Horsfield, 1829, Descriptive catalogue of the lepidopterous insects contained in the museum of the Honourable East-India Company (2): 107. TL: Java, Indonesia.

Specimen examined: 1우, BC, 18.viii.2009, Lyc.48. ㅇ, R. Lodh.

Conservation status: Tripura: occasional; IUCN/ CITES: not evaluated.

Distribution: India (Northeast, Orissa, Sikkim in the west to Arunachal Pradesh in the east in Himalaya, Tripura), Bangladesh, Bhutan, Indonesia, Myanmar, Nepal.

\section{1. *Spindasis vulcanus (Fabricius, 1775);}

Common Silverline

Papilio vulcanus Fabricius, 1775, Systema Entomologiae, sistens insectorum classes, ordines, genera, species, adiectis synonymis, locis, descriptionibus, observationibus: 519. TL: South India.

Specimen examined: 1우, DB, 7.iv.2010, Lyc.49.우, R. Lodh. 
Conservation status: Tripura: rare; IUCN/CITES: not evaluated.

Distribution: India (common in almost all states of India, Tripura), Bangladesh, Bhutan, Nepal, Pakistan, Sri Lanka.

\section{Tribe Arhopalini}

152. ${ }^{*}$ Arhopala amantes (Hewitson, 1862);

\section{Large Oak Blue}

Amblypodia amantes Hewitson, 1862, Specimens of a Catalogue of Lycaenidae in the British Museum: 4. TL: Sri Lanka.

Specimen examined: $1 \hat{\partial} / O$ (monomorphic), TWS, 18.v.2010, Lyc.32.img. ${ }^{\Uparrow} /$, R. R. Lodh.

Conservation status: Tripura: very rare; IUCN/CITES: not evaluated.

Distribution: India (peninsular India up to Gujarat and Madhya Pradesh, Tripura, Uttaranchal in the west to Arunachal Pradesh in the east in Himalaya, West Bengal), Bangladesh, Bhutan, Myanmar, Nepal, Sri Lanka.

153. *Arhopala atrax (Hewitson, 1862); Indian Oak Blue Amblypodia atrax Hewitson 1862, Specimens of a catalogue of Lycaenidae in the British Museum: 13. TL: Bengal, India.

Specimen examined: $1 \hat{\gamma} / 9$ (monomorphic), TWS, 18.v.2010, Lyc.33.img. ${ }^{\lambda} / q$, R. Lodh.

Conservation status: Tripura: very rare; IUCN/CITES: not evaluated.

Distribution: India (Chhattisgarh, Himachal Pradesh in the west to Arunachal Pradesh in the east in Himalaya, Madhya Pradesh, Orissa, Tripura, West Bengal), Bangladesh, Bhutan, Myanmar, Nepal.

\section{Arhopala centaurus (Fabricius, 1775);}

Centaur Oak Blue (Figure 37)

Papilio centaurus Fabricius, 1775, Systema Entomologiae, sistens insectorum classes, ordines, genera, species, adiectis synonymis, locis, descriptionibus, observationibus: 520. TL: Java.

Specimen examined: $10^{\lambda}$, TWS, 18.v.2010, Lyc.31. $0^{\lambda}, \mathrm{R}$. Lodh; 1요, TWS, 18.v.2010, Lyc.31. ㅇ, R. Lodh.

Conservation status: Tripura: occasional; IUCN: Near Threatened.

Distribution: India (Northeast, South India up to Gujarat, Tripura, Uttaranchal in the west to Arunachal Pradesh in the east in Himalaya, West Bengal), Bhutan, Myanmar, Nepal, Sri Lanka.

155. Arhopala fulla (Hewitson, 1862);

Spotless Oak Blue (Figure 38)

Amblypodia fulla Hewitson 1862, Specimens of a Catalogue of Lycaenidae in the British Museum: 10. TL: Buru, Inonesia.

Specimen examined: $1 \hat{\delta} /$ ( (monomorphic), TWS, 18.v.2010, Lyc.35.img. $\sigma^{\top} /$, R. Rodh.

Conservation status: Tripura: rare; India: Schedule II (IWPA).

Distribution: India (Sikkim to Arunachal Pradesh in the east in Himalaya), Bhutan, Indonesia, Myanmar.

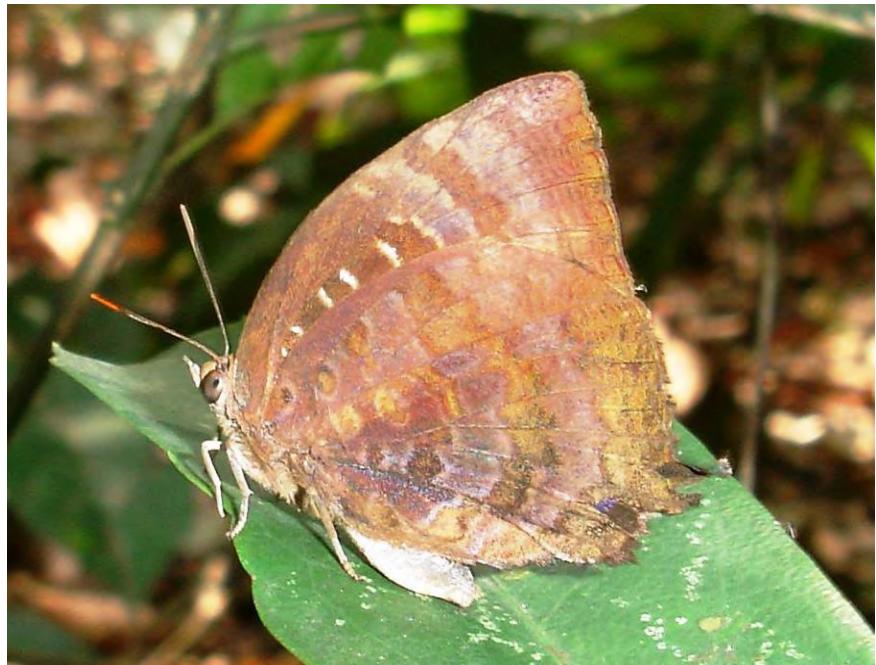

Figure 37. Arhopala centaurus.

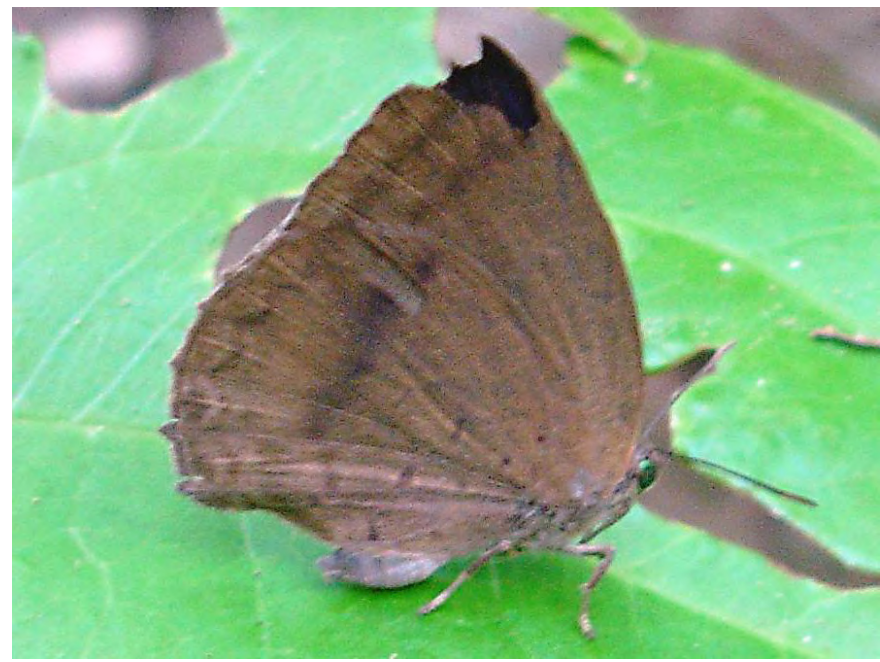

Figure 38. Arhopala fulla.

156. Arhopala selta (Hewitson, 1869); Rosy Oakblue Amblypodia selta Hewitson 1869, Illustrations of diurnal Lepidoptera. Lycaenidae (4): 14. TL: Moulmein, Myanmar.

Specimen examined: $1 \delta / \%$ (monomorphic), TWS, 18.v.2010, Lyc.34.img. $\overbrace{}^{\lambda} / Q$, R. Lodh.

Conservation status: Tripura: rare; India: Schedule I (IWPA), endangered (Red Data Book).

Distribution: India (Andaman, Northeast, Tripura), Indonesia, Myanmar, Peninsular Malaya, Thailand.

157. *Arhopala singla (de Nicéville, 1885);

Yellow-disc Oak Blue

Satadra singla de Nicéville1885, The Journal of the Asiatic Society of Bengal 54(2): 119. TL: Sikkim, India.

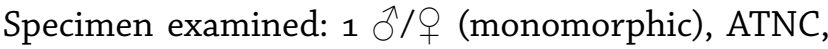
17.i.2010, Lyc.36.img. $\overbrace{}^{\lambda} /$, R. Lodh.

Conservation status: Tripura: very rare; IUCN/CITES: not evaluated.

Distribution: India (Tripura, Uttaranchal in the west to Arunachal Pradesh in the east in Himalaya), Bangladesh, Bhutan, Myanmar, Nepal. 
158. *Surendra quercetorum (Moore, 1857);

Common Acacia Blue

Amblypodia quercetorum Moore, 1857, A Catalogue of the lepidopterous insects in the museum of the Honourable East-India Company 1: 42. TL: Kangra, Himachal Pradesh, India.

Specimen examined: 10, BM, 15.x.2009, Lyc.50. $0^{\lambda}, \mathrm{R}$. Lodh.

Conservation status: Tripura: rare; IUCN/CITES: not evaluated.

Distribution: India (Jharkhand, South India up to Gujarat, Tripura, Uttaranchal eastwards to Arunachal Pradesh in Himalaya), Bangladesh, Bhutan, Myanmar, Nepal, Sri Lanka.

Tribe Catapaecilmini

159. Catapaecilma elegans (Druce, 1873);

\section{Common Tinsel}

Hypochrysops elegans Druce, 1873, Proceedings of the Zoological Society of London (2): 350. TL: Borneo, Malaysia.

Specimen examined: 10 , TWS, 18.v.2010, Lyc.37. $\hat{O}^{\hat{0}}, \mathrm{R}$. Lodh.

Conservation status: Tripura: very rare; IUCN/CITES: not evaluated.

Distribution: India [Eastern Ghats, Northeast, Tripura, Uttaranchal in the west to Arunachal Pradesh in the east in Himalaya, Western Ghats (Maharashtra southwards)], Bhutan, Myanmar, Nepal, Sri Lanka.

Tribe Cheritrini

160. ${ }^{*}$ Cheritra freja (Fabricius, 1793);

Common Imperial (Figure 39)

Hesperia freja Fabricius, 1793, Entomologia systematica 3(1): 263. TL: Nagari Hills, Andhra Pradesh, India.

Specimen examined: $1 \hat{\delta} / \phi$ (monomorphic), DB, 7.iv.2010, Lyc.38.img. ${ }^{\lambda / O}$, R. Lodh.

Conservation status: Tripura: rare; IUCN: Endangered.

Distribution: India (Tripura, Uttaranchal in the west to Arunachal Pradesh in the east in Himalaya, Western Ghats from Maharashtra southwards), Bangladesh, Bhutan, Myanmar, Nepal, Sri Lanka.

Tribe Hypolycaenini

161. *Hypolycaena erylus (Godart, 1824); Common Tit Polyommatus erylus Godart, 1824, Encyclopédie méthodique 9(2): 600, 633. TL: Java, Indonesia.

Specimen examined: 10, BC, 19.v.2011, Lyc.39. ${ }^{\lambda}, \mathrm{R}$. Lodh; 1ㅇ, BC, 19.v.2011, Lyc.39. 오, R. Lodh.

Conservation status: Tripura: common; IUCN/CITES: not evaluated.

Distribution: India (Andaman, Northeast, Tripura, Sikkim in the west to Arunachal Pradesh in the east in Himalaya), Bangladesh, Bhutan, Indonesia, Myanmar, Nepal.

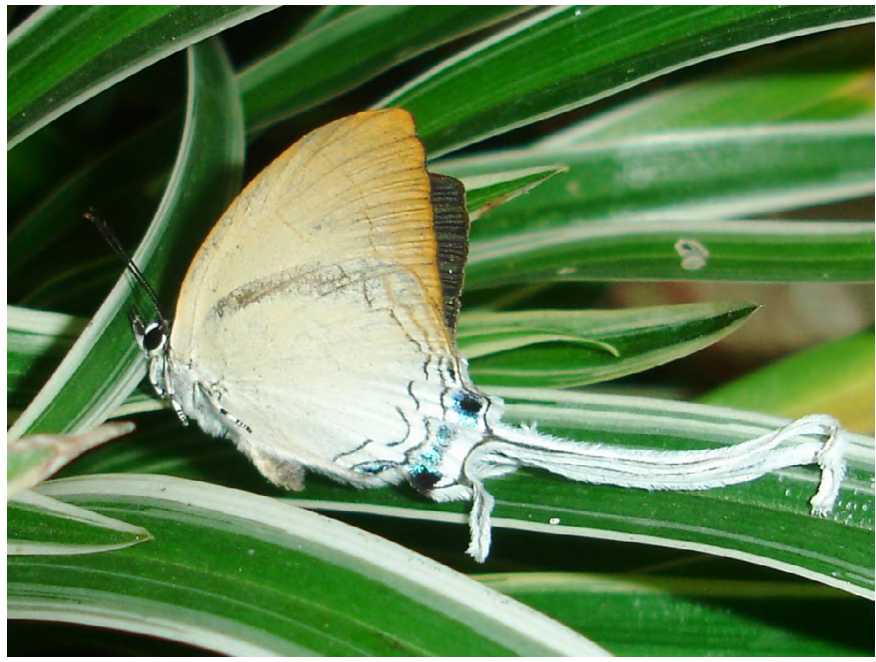

Figure 39. Cheritra freja.

162. *Zeltus amasa (Hewitson, 1865); Fluffy Tit

Hypolycaena amasa Hewitson, 1865, Illustrations of diurnal Lepidoptera. Lycaenidae (2): 51. TL: India.

Specimen examined: $1 \delta / q$ (monomorphic), DB, 14.v.2010, Lyc.51.img. ${ }^{\lambda} / q$, R. Lodh.

Conservation status: Tripura: rare; IUCN/CITES: not evaluated.

Distribution: India [Northeast, Sikkim to Arunachal Pradesh in the east in Himalaya, Tripura, Western Ghats (Goa southwards)], Bangladesh, Bhutan, Myanmar, Nepal.

Tribe Loxurini

163. Loxura atymnus (Cramer, 1782); Yamfly Papilio atymnus Cramer, 1782, Uitlandsche Kapellen (Papillons exotiques) 4(26b-28): 82. TL: South India.

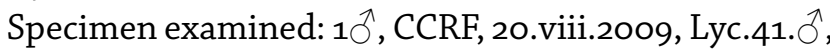
R. Lodh; 19, CCRF, 20.viii.2009, Lyc.41., , R. Lodh.

Conservation status: Tripura: common; IUCN/CITES: not evaluated.

Distribution: India (peninsular India up to Maharashtra, south Bihar to Madhya Pradesh, Tripura, Uttaranchal in the west to Arunachal Pradesh in the east in Himalaya, West Bengal), Bangladesh, Bhutan, Myanmar, Nepal.

Tribe Deudorigini

164. *Rapala iarbus (Fabricius, 1787); Indian Red Flash Papilio iarbus Fabricius, 1787, Mantissa Insectorum 2: 68. TL: Thailand.

Specimen examined: 1ㅇ, MB, 16.xii.2009, Lyc.43.. , R. Lodh.

Conservation status: Tripura: very rare; IUCN/CITES: not evaluated.

Distribution: India (Northeast, Orissa, Sikkim in the west to Arunachal Pradesh in the east in Himalaya, Tripura, West Bengal), Bangladesh, Bhutan, Myanmar, Nepal, Thailand. 
165. *Rapala manea (Hewitson, 1863); Slate Flash

(Figure 40)

Deudorix manea Hewitson, 1863, Illustrations of diurnal Lepidoptera. Lycaenidae (1): 23. TL: Sulawesi, Indonesia.

Specimen examined: $1 \hat{\delta} / 9$ (monomorphic), TWS, 18.v.2010, Lyc.44.img. $\widehat{\jmath} /$,, R. Lodh.

Conservation status: Tripura: rare; IUCN: vulnerable.

Distribution: India (common in almost all states of India except in arid zone, Tripura), Bangladesh, Bhutan, Indonesia, Myanmar, Nepal, Pakistan, Sri Lanka.

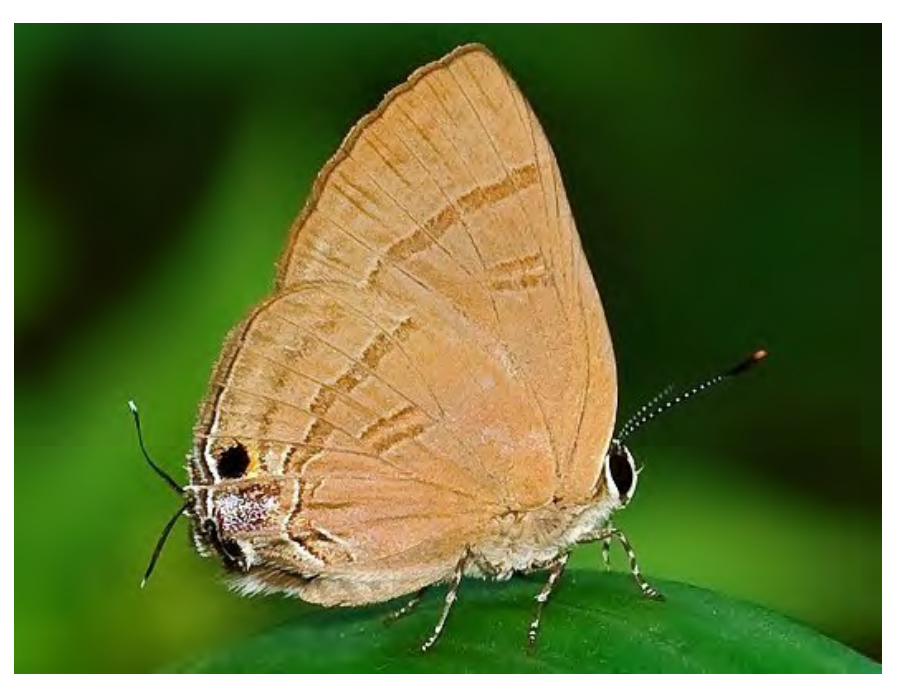

Figure 40. Rapala manea.

166. ${ }^{*}$ Rapala pheretima (Hewitson, 1863);

Copper Flash

Deudorix pheretima Hewitson, 1863, Illustrations of diurnal Lepidoptera. Lycaenidae (1): 21. TL: Sarawak, Malaysia.

Specimen examined: $1 \hat{\delta} / 9$ (monomorphic), TWS, 18.v.2010, Lyc.42.img. $\hat{\jmath} /$, , R. Lodh.

Conservation status: Tripura: rare; IUCN/CITES: not evaluated.

Distribution: India (Northeast, Orissa, Sikkim in the west to Arunachal Pradesh in the east in Himalaya, Tripura, West Bengal), Bangladesh, Bhutan, Malaysia, Myanmar, Nepal.

167. *Rapala suffusa (Moore, 1878); Suffused Flash Deudorix suffusa Moore, 1878, Proceedings of the Zoological Society of London (4): 834. TL: Taco, Upper Tenasserim, Myanmar.

Specimen examined: $1 \hat{\delta} / 9$ (monomorphic), DB, 7.iv.2010, Lyc.45.img. $\jmath^{\lambda} / Q$, R. Lodh.

Conservation status: Tripura: very rare; IUCN/CITES: not evaluated.

Distribution: India, (Assam, north India, Tripura), China, Myanmar, Thailand.

Tribe Remelanini

168. *Remelana jangala (Horsfield, 1829);

Chocolate Royal

Amblypodia jangala Horsfield, 1829, Descriptive catalogue of the lepidopterous insects contained in the museum of the Honourable East-
India Company (2): 113. TL: Java, Indonesia.

Specimen examined: $10^{\lambda}, \mathrm{BM}, 15 \cdot$ X.2009, Lyc.46. $0^{\lambda}, \mathrm{R}$. Lodh.

Conservation status: Tripura: rare; IUCN/CITES: not evaluated.

Distribution: India (Northeast, Sikkim to Arunachal Pradesh in the east in Himalaya, Tripura), Bangladesh, Bhutan, Indonesia, Myanmar, Nepal.

Family Riodinidae

Subfamily Nemeobiinae

Tribe Zemerini

169. Zemeros flegyas (Cramer, 1780); Punchinello Papilio flegyas Cramer, 1780, Uitlandsche Kapellen (Papillons exotiques) 3(23-24): 158. TL: China.

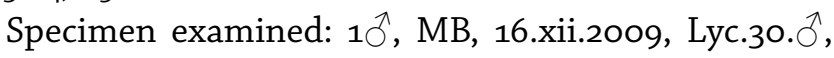
R. Lodh; 1이 MB, 16.xii.2009, Lyc.30.ㅇ, R. Lodh.

Conservation status: Tripura: very common; IUCN/ CITES: not evaluated.

Distribution: India (Northeast, Uttaranchal in the west to Arunachal Pradesh in the east in Himalaya, Tripura), Bangladesh, Bhutan, China, Myanmar, Nepal.

Family Hesperiidae

Subfamily Coeliadinae

\section{Badamia exclamationis (Fabricius, 1775);}

Brown Awl (Figure 41)

Papilio exclamationis Fabricius, 1775, Systema Entomologiae, sistens insectorum classes, ordines, genera, species, adiectis synonymis, locis, descriptionibus, observationibus: 530. TL: South India.

Specimen examined: 10, JRF, 16.viii.2009, Hes.2. $\hat{0}^{\lambda}$, R. Lodh; 1 , JRF, 16.viii.2009, Hes.2.b. 9 , R. Lodh.

Conservation status: Tripura: rare; IUCN: vulnerable.

Distribution: India (common in almost all states of India, Tripura), Afghanistan, Bangladesh, Bhutan, Myanmar, Nepal, Pakistan, Sri Lanka.

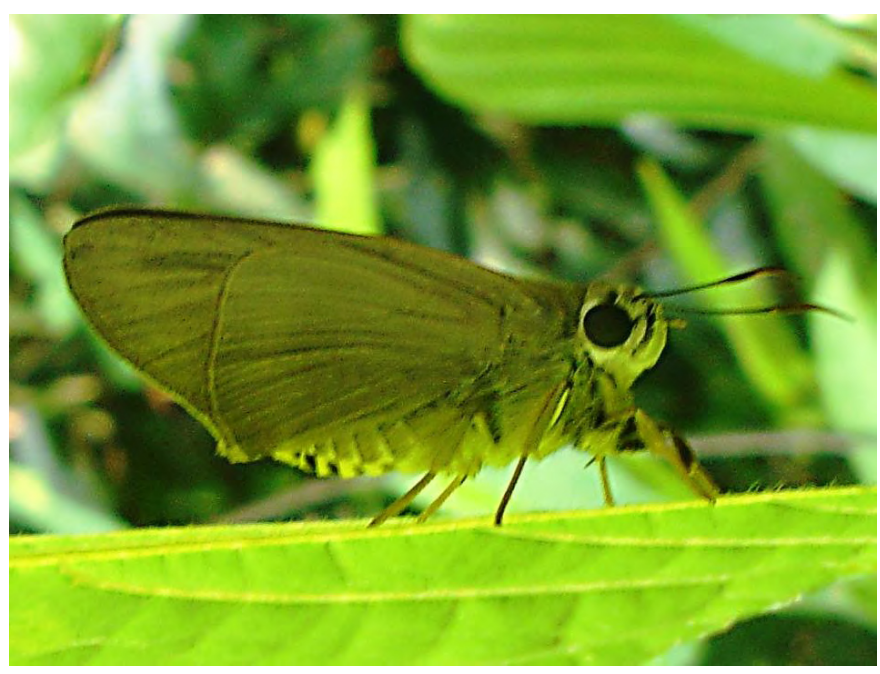

Figure 41. Badamia exclamationis. 
171. *Bibasis oedipodea (Swainson, 1820);

Branded Orange Awlet

Ismene oedipodea Swainson, 1820, Zoological illustrations (1): 16. TL: Java, Indonesia.

Specimen examined: 19, DB, 14.v.2010, Hes.1. 9, R. Lodh.

Conservation status: Tripura: very rare; IUCN/CITES: not evaluated.

Distribution: India (Northeast, Himachal Pradesh, Tripura, Uttaranchal in the west to Arunachal Pradesh in the east in Himalaya), Bangladesh, Bhutan, Indonesia, Myanmar, Nepal, Sri Lanka.

172. *Choaspes benjaminii (Guérin-Méneville, 1843);

Indian Awlking (Figure 42)

Hesperia benjaminii Guérin-Méneville 1843 in Delessert, Souvenirs d'un voyage dans l'Inde 2(2): 79. TL: Nilgiris, South India.

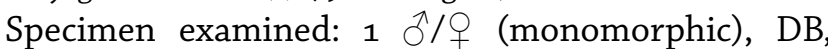
14.v.2010, Hes.3.img. $\sigma^{\top} / \phi$, R. Lodh.

Conservation status: Tripura: very rare; IUCN: Near Threatened.

Distribution: India [Himachal Pradesh in the west to Arunachal Pradesh in the east in Himalaya, Northeast, South India (Karnataka southwards), Tripura], Afghanistan, Bangladesh, Bhutan, Nepal, North Myanmar, Sri Lanka.

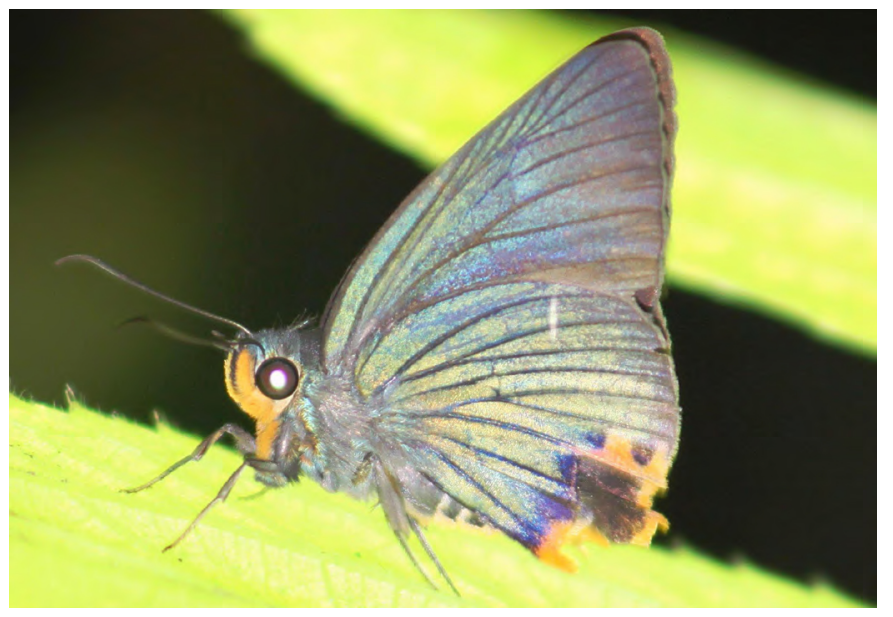

Figure 42. Choaspes benjaminii.

Subfamily Hesperiinae

Tribe Aeromachini

173. *Aeromachus pygmaeus (Fabricius, 1775); Pygmy Scrub Hopper

Papilio pygmaeus Fabricius, 1775, Systema Entomologiae, sistens insectorum classes, ordines, genera, species, adiectis synonymis, locis, descriptionibus, observationibus: 536. TL: Koenig, South India.

Specimen examined: $1 \delta^{\lambda}, \mathrm{DB}$, 7.iv.2010, Hes.7.img. $\lesssim / O+$, R. Lodh.

Conservation status: Tripura: rare; IUCN/CITES: not evaluated.

Distribution: India [Sikkim to Arunachal Pradesh in the east in Himalaya, Tripura, Western Ghats (Maharashtra southwards)], Myanmar, Sri Lanka.
174. ${ }^{*}$ Ampittia dioscorides (Fabricius, 1793);

\section{Bush Hopper}

Hesperia dioscorides Fabricius, 1793, Entomologia systematica 3 (1): 329. TL: Tranquebar, South India.

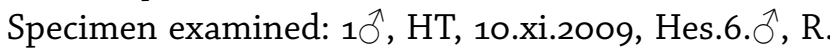
Lodh; 1 , DB, 14.v.2010, Hes.6. 9 , R. Lodh.

Conservation status: Tripura: occasional; IUCN/ CITES: not evaluated.

Distribution: India (peninsular India, Sikkim to Northeast, Tripura, West Bengal), Bangladesh, Myanmar, Nepal, Sri Lanka.

\section{Ancistroides nigrita (Latreille, 1824);}

\section{Chocolate Demon}

Hesperia nigrita Latreille, 1824, Encyclopédie Méthodique 9(2): 788. TL: Java, Indonesia.

Specimen examined: $1 \widehat{\jmath}$, SWS, 14.ii.2010, Hes.5.img. $\overbrace{}^{\jmath}$, R. Lodh.

Conservation status: Tripura: occasional; IUCN/CITES: not evaluated.

Distribution: India [Northeast, Tripura, Uttaranchal in the west to Arunachal Pradesh in the east in Himalaya, West Bengal (Darjeeling region)], Bangladesh, Bhutan, Myanmar, Nepal.

176. *Arnetta vindhiana (Moore, 1883); Vindayan Bob Isoteinon vindhiana Moore, 1883, Proceedings of the Zoological Society of London (2): 533. TL: India.

Specimen examined: $1 \hat{\sigma} / q$ (monomorphic), DB, 7.iv.2010, Hes.4.img. $\lesssim / O$, R. Lodh.

Conservation status: Tripura: very rare; IUCN/CITES: not evaluated.

Distribution: India (Gujarat and Madhya Pradesh, South India, Tripura).

177. *Cupitha purreea (Moore, 1877); Wax Dart Pamphila purreea Moore, 1877, Proceedings of the Zoological Society of London (3): 594. TL: Andaman, India.

Specimen examined: $1 \hat{\jmath}$, ATNC, 12.iv.2012, Hes.10. $\widehat{\jmath}$, R. Lodh; 1 으, ATNC, 12.iv.2012, Hes.10.ㅇ, R. Lodh.

Conservation status: Tripura: very rare; IUCN/CITES: not evaluated.

Distribution: India [Andaman, Sikkim to further Northeast, South India (Karnataka southwards), Tripura], Bangladesh, Bhutan, Myanmar, Nepal.

\section{8. *Gangara thyrsis (Fabricius, 1775); Giant Redeye}

\section{(Figure 43)}

Papilio thyrsis Fabricius, 1775, Systema Entomologiae, sistens insectorum classes, ordines, genera, species, adiectis synonymis, locis, descriptionibus, observationibus: 532. TL: "America" (error for Tranquebar, South India).

Specimen examined: $1 \hat{\jmath}$, TWS, 18.v.2010, Hes.12. $\widehat{O}$, R. Lodh; 19 , TWS, 18.v.2010, Hes.12. 9 , R. Lodh.

Conservation status: Tripura: rare; near threatened (IUCN).

Distribution: India (Andaman, South India [southwards of Mumbai], Sikkim to Northeast, Tripura, West 


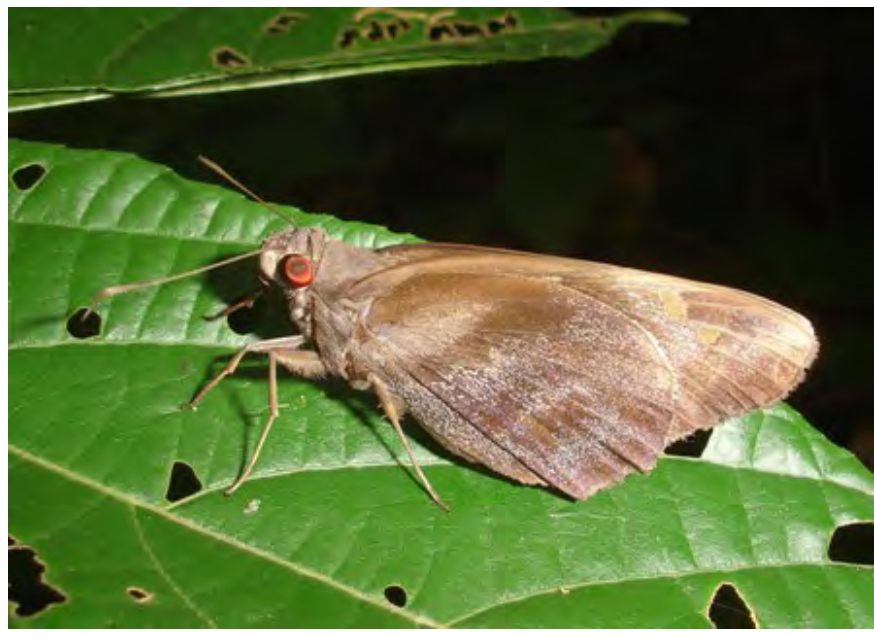

Figure 43. Gangara thyrsis.

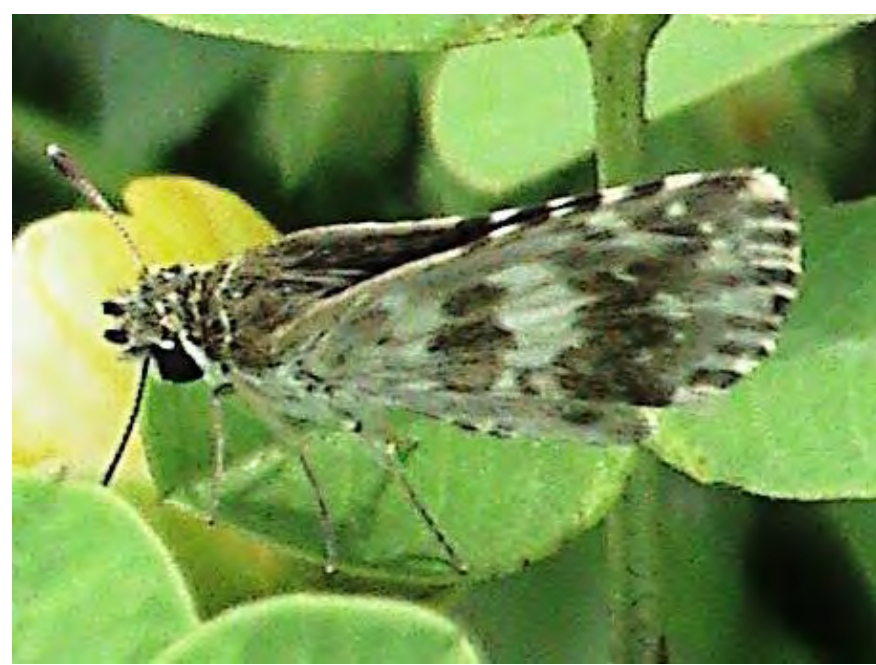

Figure 44. Halpe homolea.

Bengal), Bangladesh, Myanmar, Nepal, Sri Lanka.

179. ${ }^{*}$ Halpe homolea (Hewitson, 1868); Indian Ace (Figure 44)

Hesperia homolea Hewitson, 1868, Descriptions of one Hundred new Species of Hesperidae (2): 29. TL: Singapore.

Specimen examined: 1ð̂, ATNC, 15.iv.2011, Hes.14. img. $\circlearrowleft /$,, R. Lodh.

Conservation status: Tripura: rare; India: Schedule II (IWPA).

Distribution: India [Sikkim to Arunachal Pradesh in the east in Himalaya, Tripura, Western Ghats (Maharashtra southwards)], Myanmar, Singapore, Sri Lanka.

180. *Halpe porus (Mabille, 1876); Moore's Ace

Hesperilla porus Mabille, 1876, Annales de la Société entomologique de France (5)6: 39. TL: Assam, India.

Specimen examined: $1 \hat{\sigma} / \phi$ (monomorphic), ATNC, 15.iv.2011, Hes.15.img. $\widehat{\sigma} / \phi$, R. Lodh.

Conservation status: Tripura: rare; IUCN/CITES: not evaluated.

Distribution: India [Andaman, South Bihar, Tripura, Uttaranchal (Mussoorie) to Northeast, Western Ghats
(Maharashtra southwards)], Bhutan, Myanmar, Nepal.

181. *Halpe zema (Hewitson, 1877); Banded Ace

Hesperia zema Hewitson 1877, Annals and Magazine of Natural History (4) 19 (109): 77. TL: Sikkim, India.

Specimen examined: $10 \hat{\sigma}$, ATNC, 15.iv.2011, Hes.16. img. $\widehat{\jmath} / q$, R. Lodh.

Conservation status: Tripura: very rare; IUCN/CITES: not evaluated.

Distribution: India (Darjeeling-Sikkim in the west to Arunachal Pradesh in the east in Himalaya, Northeast), Myanmar.

182. ${ }^{*}$ Hyarotis adrastus (Stoll, 1782); Tree Flitter Papilio adrastus Stoll, 1782, Uitlandsche Kapellen (Papillons exotiques) 4(26b-28): 62. TL: "Surinam" (error for Sri Lanka).

Specimen examined: $1 \hat{\sigma} / q$ (monomorphic), DB, 7.iv.2010, Hes.13.img. $\lesssim / q$, R. Lodh.

Conservation status: Tripura: very rare; India: Schedule IV (IWPA).

Distribution: India [Himachal Pradesh in the west to Arunachal Pradesh in the east in Himalaya, Northeast, Tripura, Western Ghats (South of Goa), West Bengal], Bangladesh, Bhutan, Myanmar, Nepal, Sri Lanka.

183. Iambrix salsala (Moore, 1866); Chestnut Bob Nisoniades salsala Moore, 1866, Proceedings of the Zoological Society of London (3): 786. TL: Sikkim, India.

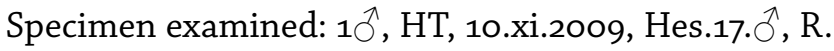
Lodh; 1ㅇ, HT, 10.xi.2009, Hes.17.b.ㅇ, R. Lodh.

Conservation status: Tripura: occasional; IUCN/ CITES: not evaluated.

Distribution: India (common in almost all states of India, Tripura), Bangladesh, Bhutan, Myanmar, Nepal, Sri Lanka.

184. *Koruthaialos butleri (de Nicéville, 1883);

Dark Velvet Bob

Astictopterus butleri de Nicéville, 1883, The Journal of the Asiatic Society of Bengal 52(2/4): 98. TL: Sikkim, India.

Specimen examined: $1 \hat{\jmath}, \mathrm{DB}$, 7.iv.2010, Hes.19. ${ }^{\lambda}, \mathrm{R}$. Lodh; 1 , DB, 7.iv.2010, Hes.19. 9 , R. Lodh.

Conservation status: Tripura: rare; IUCN/CITES: not evaluated.

Distribution: India (Northeast, Sikkim in the west to Arunachal Pradesh in the east in Himalaya, Tripura), Bhutan, Myanmar, Nepal.

185. *Koruthaialos sindu (C. Felder \& R. Felder, 1860); Bright Red Velvet Bob

Astictopterus sindu C. Felder \& R. Felder, 1860, Wiener entomologische Monatschrift 4(12): 401. TL: Malaysia.

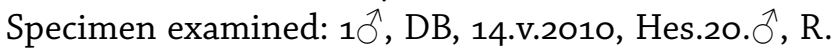
Lodh; 1 + , DB, 14.v.2010, Hes.20. 9 , R. Lodh.

Conservation status: Tripura: rare; IUCN/CITES: not evaluated.

Distribution: India (Assam to Arunachal Pradesh, 
Northeast, Tripura), Malaysia, Myanmar.

186. ${ }^{*}$ Matapa aria (Moore, 1865); Common Redeye Ismene aria Moore, 1865, Proceedings of the Zoological Society of London (3): 784. TL: Bengal, India.

Specimen examined: $10^{\lambda}, \mathrm{BM}, 15 \cdot \mathrm{X} \cdot 2009$, Hes.21. $0^{\lambda}, \mathrm{R}$. Lodh; 1ㅇ, BM, 15.x.2009, Hes.21. , R. Lodh.

Conservation status: Tripura: occasional; IUCN/ CITES: not evaluated.

Distribution: India [Andaman, South India (up to Mumbai), Tripura, Uttaranchal in the west to Northeast in the east in Himalaya, West Bengal], Bangladesh, Myanmar, Nepal, Sri Lanka.

187. ${ }^{*}$ Notocrypta curvifascia (C. Felder \& R. Felder, 1862); Restricted Demon (Figure 45)

Plesioneura curvifascia C. Felder \& R. Felder, 1862, Wiener entomologische Monatschrift 6(1): 29. TL: Ningpo, China.

Specimen examined: $10^{\hat{O}}, \mathrm{MR}$, 13.v.2010, Hes.22. ${ }^{\lambda}, \mathrm{R}$. Lodh; 1ㅇ, MR, 13.v.2010, Hes.22. ㅇ, R. Lodh.

Conservation status: Tripura: rare; IUCN: vulnerable.

Distribution: India [Andaman, Himachal Pradesh in the west to Arunachal Pradesh in the east in Himalaya, Northeast, Tripura Western Ghats (south of Goa)], Bangladesh, Bhutan, Myanmar, Nepal, Sri Lanka.

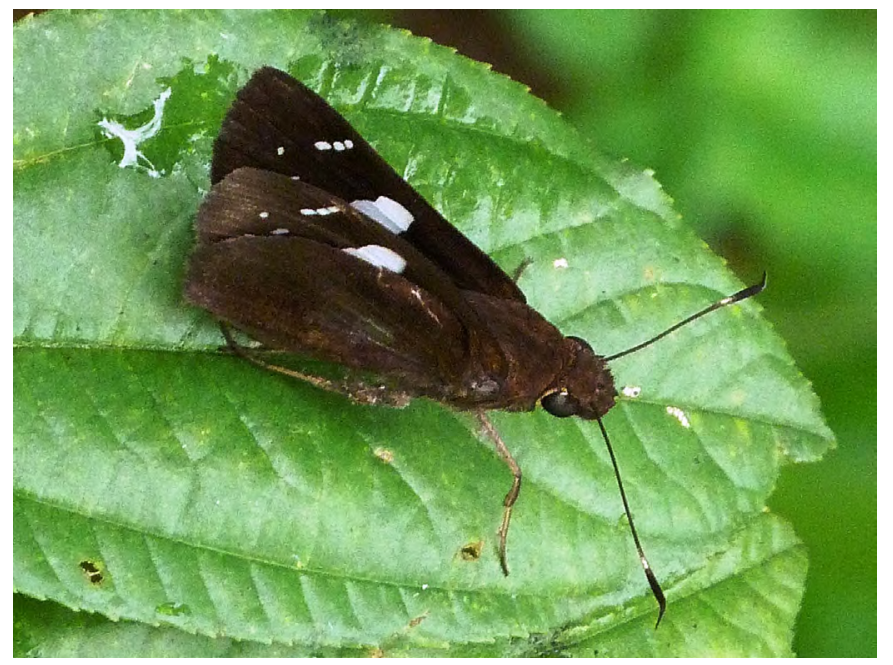

Figure 45, Notocrypta curvifascia.

188. *Notocrypta feisthamelii (Boisduval, 1832);

Himalayan Spotted Demon

Thymele feisthamelii Boisduval 1832, in d'Urville, Voyage de decouvertes de l'Astrolabe 1: 159. TL: Amboina, Indonesia.

Specimen examined: $1 \hat{\delta} / \phi$ (monomorphic), DB, 7.iv.2010, Hes.24.img. $\overbrace{}^{\lambda} / Q$, R. Lodh.

Conservation status: Tripura: very rare; IUCN/CITES: not evaluated.

Distribution: India (Jammu and Kashmir in the west to Arunachal Pradesh in the east in Himalaya, Northeast), Bangladesh, Bhutan, Indonesia, Myanmar, Nepal, Sri Lanka.

189. *Notocrypta paralysos (Wood-Mason \& de Nicéville, 1881); Common Banded Demon
Plesioneura paralysos Wood-Mason and de Nicéville 1881, Proceedings of the Asiatic Society of Bengal: 143. TL: South Andaman Islands.

Specimen examined: $1 \hat{O}^{\hat{n}}$, SWS, 14.ii.2010, Hes.23. img. $\hat{\jmath}$, R. Lodh.

Conservation status: Tripura: very rare; IUCN/CITES: not evaluated.

Distribution: India (Andaman, Himachal Pradesh in the west to Arunachal Pradesh in the east in Himalaya, Northeast, Tripura, Western Ghats south of Goa), Bangladesh, Bhutan, Myanmar, Nepal, Sri Lanka.

\section{0. *Sebastonyma dolopia (Hewitson, 1868);}

\section{Tufted Ace}

Hesperia dolopia Hewitson, 1868, Descriptions of One Hundred New Species of Hesperidae (2): 27. TL: Sikkim, India.

Specimen examined: $1 \delta / Q$ (monomorphic), CCRF, 20.viii.2009, Hes.31.img. $\hat{0} /$, , R. Lodh.

Conservation status: Tripura: rare; IUCN/CITES: not evaluated.

Distribution: India (Sikkim in the west to Arunachal Pradesh in the east in Himalaya), Bhutan, Myanmar, Nepal.

191. *Suada swerga (de Nicéville, 1883); Grass Bob

Hesperia swerga de Nicéville, 1883, The Journal of the Asiatic Society of Bengal: 89. TL: Sikkim, India.

Specimen examined: 10 , DB, 7.iv.2010, Hes.30.img. ${ }^{\hat{n}}$, R. Lodh; 1ㅇ, DB, 7.iv.2010, Hes.30.우, R. Lodh.

Conservation status: Tripura: very rare; IUCN/CITES: not evaluated.

Distribution: India (common in almost all states of India, Tripura), Java, Laos, Malaysia, Myanmar, South Vietnam, Sri Lanka, Thailand.

192. Suastus gremius (Fabricius, 1798); Indian Palm Bob Hesperia gremius Fabricius, 1798, Supplementum Entomologiae Systematicae: 433. TL: India.

Specimen examined: 10, DB, 7.iv.2010, Hes.29.img. ${ }^{\lambda}$, R. Lodh; 1ㅇ, ATNC, 12.iv.2012, Hes.29. 우, R. Lodh.

Conservation status: Tripura: rare; IUCN/CITES: not evaluated.

Distribution: India (common in almost all states of India, Tripura), Bangladesh, Bhutan, Myanmar, Nepal, Pakistan, Sri Lanka.

\section{Udaspes folus (Cramer, 1775); Grass Demon}

(Figure 46)

Papilio folus Cramer, 1775, Uitlandsche Kapellen (Papillons exotiques) 1 (1-7): 118. TL: Surinam.

Specimen examined: 10 , SS, 13.ii.2010, Hes.34. $0^{\Uparrow}, \mathrm{R}$. Lodh; 19, SS, 13.ii.2010, Hes.34. , R. Lodh.

Conservation status: Tripura: common; IUCN: Endangered.

Distribution: India (Himachal Pradesh in the west to Arunachal Pradesh in the east in Himalaya, Madhya Pradesh, Northeast, South India up to south Gujarat, Tripura, Uttar Pradesh), Bangladesh, Bhutan, Myanmar, Nepal, Sri Lanka, Surinam. 


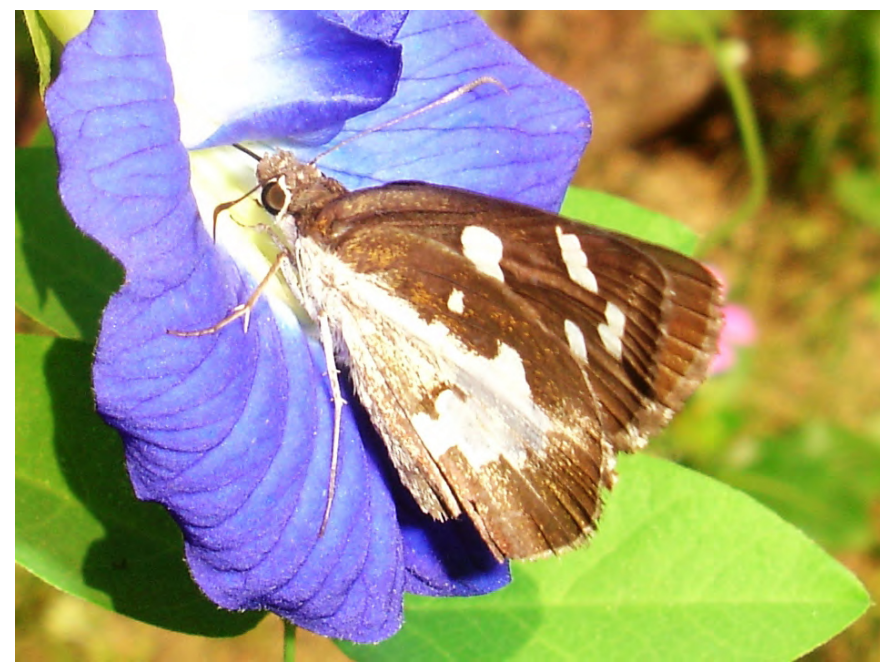

Figure 46. Udaspes folus.

Tribe Baorini

194. Baoris farri (Moore, 1878); Paintbrush Swift

Hesperia farri Moore, 1878, Proceedings of the Zoological Society of London (3): 688. TL: Calcutta, Cherrapunji, India.

Specimen examined: 10 , SWS, 6.viii.2009, Hes.9.img. $\widehat{J} / 9$, R. Lodh.

Conservation status: Tripura: very rare; India: Schedule IV (IWPA), IUCN: Critically Endangered.

Distribution: India (Andaman and Central Nicobar Island, further Northeast, Jharkhand, Tripura, Uttaranchal in the west to Sikkim in the east in Himalaya, Western Ghats south of Goa, West Bengal), Bangladesh, Bhutan, Myanmar, Nepal, Sri Lanka.

195. *Borbo cinnara (Wallace, 1866); Rice Swift

Hesperia cinnara Wallace, 1866, Proceedings of the Zoological Society of London (2): 361. TL: Formosa, China.

Specimen examined: $1 \hat{\delta} / \phi$ (monomorphic), RWS, 19.viii.2009, Hes.8.img. $§ /$, R. Lodh.

Conservation status: Tripura: rare; IUCN: Near Threatened.

Distribution: India (common in almost all states of India, Tripura), Bangladesh, Bhutan, China, Myanmar, Nepal, Sri Lanka.

196. ${ }^{*}$ Caltoris kumara (Moore, 1878); Blank Swift

Hesperia kumara Moore, 1878, Proceedings of the Zoological Society of London (3): 687. TL: North Karnataka, India.

Specimen examined: $1 \hat{\delta} / 9$ (monomorphic), DB, 7.iv.2010, Hes.11.img. ${ }^{\top} / Q$, R. Lodh.

Conservation status: Tripura: rare; IUCN/CITES: not evaluated.

Distribution: India (Sikkim, Tripura, Western Ghats from Goa southwards, West Bengal), Bangladesh, Myanmar, Sri Lanka.

197. ${ }^{*}$ Iton semamora (Moore, 1865);

Bengal Common Wight
Hesperia semamora Moore, 1865, Proceedings of the Zoological Society of London (3): 791. TL: Bengal, India.

Specimen examined: 10, BC, 19.v.2011, Hes.18.img. $\pi / Q$, R. Lodh.

Conservation status: Tripura: very rare; IUCN/CITES: not evaluated.

Distribution: India (common in almost all states of India, Tripura.), Sri Lanka, Banggai, Borneo, China, Java, Lesser Sunda Is., Malayan Peninsula, Palawan Peninsula, Philippines, Sangihe, Sumatra, Sulawesi, Sula, Talaud Is., Tukangbesi.

198. Parnara guttatus (Bremer \& Grey, 1853);

Straight Swift

Eudamus guttatus Bremer \& Grey, 1853, Diagnoses de Lepidopteres Nouveaux 1: 6o. TL: Beijing, China.

Specimen examined: 1이, DB, 14.v.2010, Hes.26.img. 우, R. Lodh.

Conservation status: Tripura: rare; IUCN/CITES: not evaluated.

Distribution: India (common $\mathrm{n}$ almost all states of India except in South India, Tripura), Bangladesh, Bhutan, China, Myanmar, Nepal, Pakistan, Sri Lanka.

199. *Pelopidas agna (Moore, 1865);

Obscure Branded Swift

Hesperia agna Moore, 1865, Proceedings of the Zoological Society of London (3): 791. TL: Bengal, India.

Specimen examined: 10, BC, 19.v.2011, Hes.28. img. $\hat{\jmath} / 9$, R. Lodh.

Conservation status: Tripura: rare; IUCN/CITES: not evaluated.

Distribution: India (common in almost all states of India, Tripura), Banggai, Borneo,China, Java,Lesser Sunda Is., Malay Peninsula, Palawan Peninsula, Philippines, Sangihe, Sri Lanka, Sulawesi, Sula, Sumatra, Talaud Is., Tukangbesi.

200. *Pelopidas mathias (Fabricius, 1798);

Small Branded Swift

Hesperia mathias Fabricius, 1798, Supplementum Entomologiae Systematicae: 433. TL: Tranquebar, South India.

Specimen examined: $1 \delta / \phi$ (monomorphic), DB, 7.iv.2010, Hes.27.img. ${ }^{\Uparrow} /$, , R. Lodh.

Conservation status: Tripura: rare; IUCN/CITES: not evaluated.

Distribution: India (common in almost all states of India, Tripura), Bangladesh, Bhutan, Myanmar, Nepal, Sri Lanka.

Tribe Taractrocerini

201. *Potanthus dara (Kollar, 1842); Himalayan Dart Hesperia dara Kollar 1842, in Hügel, Kaschmir und das Reich der Siek 4: 455. TL: Himalaya.

Specimen examined: 1 $\delta / \phi$ (monomorphic), ATNC, 12.iv.2012, Hes.25. $\sigma^{1} / Q$, R. Lodh. 
Conservation status: Tripura: very rare; IUCN/CITES: not evaluated.

Distribution: India [Andaman, Northeast, Sikkim, Tripura, Uttaranchal in the west to Arunachal Pradesh in the east in Himalaya, Western Ghats (Karnataka southwards)], Bhutan, Myanmar, Nepal, Sri Lanka.

202. ${ }^{*}$ Telicota ancilla (Herrich-Schäffer, 1869);

Dark Palm Dart

Pamphila ancilla Herrich-Schäffer, 1869, Entomologischer Verein zu Stettin 30(1-3): 79. TL: Rockhampton, Australia.

Specimen examined: 1 ऊ/ $/$ (monomorphic), ATNC, 12.iv.2012, Hes.32.img. $\hat{\jmath} /$, , R. Lodh.

Conservation status: Tripura: rare; IUCN: vulnerable.

Distribution: India (common in almost all states of India, Tripura), Australia, Bangladesh, Bhutan, Myanmar, Nepal, Pakistan, Sri Lanka.

203. *Telicota colon (Fabricius, 1775); Pale Palm Dart Papilio colon Fabricius, 1775, Systema Entomologiae, sistens insectorum classes, ordines, genera, species, adiectis synonymis, locis, descriptionibus, observationibus: 531. TL: India.

Specimen examined: 10, MB, 16.xii.2009, Hes.33. $\hat{0}^{\lambda}$, R. Lodh; 1ㅇ, ATNC, 12.iv.2012, Hes.33.ㅇ, R. Lodh.

Conservation status: Tripura: occasional; IUCN/ CITES: not evaluated.

Distribution: India (common in almost all states of India), Bangladesh, Bhutan, Myanmar, Nepal, Pakistan, Sri Lanka.

Subfamily Pyrginae

Tribe Celaenorrhinini

204. * Celaenorrhinus aurivittata (Moore, 1878); Dark Yellow-banded Flat

Plesioneura aurivittata Moore, 1878, Proceedings of the Zoological Society of London (4): 843. TL: Meetan, Myanmar.

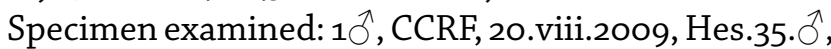
R. Lodh; 19, CCRF, 20.viii.2009, Hes.35. 9 , R. Lodh.

Conservation status: Tripura: very rare; IUCN/CITES: not evaluated.

Distribution: India (Assam eastwards up to Arunachal Pradesh, Tripura), Bangladesh, Myanmar.

205. *Pseudocoladenia dan (Fabricius, 1787);

Fulvous Pied Flat

Papilio dan Fabricius, 1787, Mantissa Insectorum 2: 88. TL: Tranquebar, South India.

Specimen examined: $1 \hat{0}, \mathrm{DB}$, 7.iv.2010, Hes.38. ${ }^{\lambda}, \mathrm{R}$. Lodh; 1ㅇ, DB, 7.iv.2010, Hes.38. ㅇ, R. Lodh.

Conservation status: Tripura: rare; IUCN/CITES: not evaluated.

Distribution: India [Himachal Pradesh in the west to Arunachal Pradesh in the east in Himalaya, South India (up to Gujarat), Tripura], Bangladesh, Bhutan, Myanmar, Nepal.

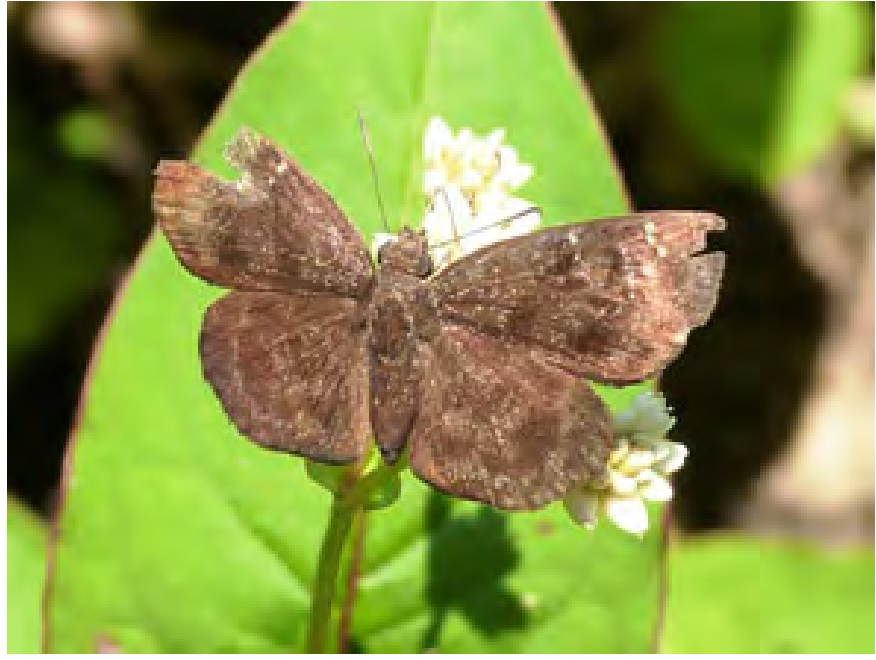

Figure 47. Sarangesa dasahara.

206. Sarangesa dasahara Moore, 1865

Common Small Flat (Figure 47)

Sarangesa dasahara Moore, 1865, Proceedings of the Zoological Society of London (3): 787. TL: Bengal, India.

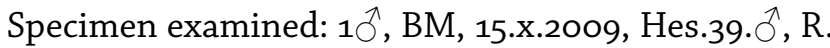
Lodh; 1ㅇ, BM, 15.X.2009, Hes.39. 오, R. Lodh.

Conservation status: Tripura: occasional; IUCN: Near Threatened.

Distribution: India (common in almost all states of India except in drier Northwest, Tripura), Bangladesh, Myanmar, Nepal, Sri Lanka.

207. *Sarangesa purendra Moore, 1882;

Spotted Small Flat

Sarangesa purendra Moore, 1882, Proceedings of the Zoological Society of London (1): 262. TL: Asia.

Specimen examined: $1 \hat{\delta} / Q$ (monomorphic), BM, 15.x.2009, Hes.40.img. ${ }^{\lambda} / q$, R. Lodh.

Conservation status: Tripura: rare; IUCN/CITES: not evaluated.

Distribution: India (Himachal Pradesh in the west to Uttaranchal in the east in Himalaya, Madhya Pradesh, South India up to Kutch in Gujarat, Tripura), Nepal, Pakistan.

Tribe Tagiadini

208. *Gerosis phisara (Moore, 1884); Dusky Yellow-

Breasted Flat

Satarupa phisara Moore 1884, The Journal of the Asiatic Society of Bengal (1): 50. TL: Khasi Hills, India.

Specimen examined: 10 , CCRF, 20.viii.2009, Hes.36. $\partial^{\lambda}$, R. Lodh.

Conservation status: Tripura: very rare; IUCN/CITES: not evaluated.

Distribution: India (Himachal Pradesh in the west to Arunachal Pradesh in the east in Himalaya, Northeast, Tripura), Bangladesh, Bhutan, Myanmar, Nepal. 
209. ${ }^{*}$ Odontoptilum angulata (Felder, 1862);

Chestnut Angle

Pterygospidea angulata Felder, 1862, Verhandlungen der Zoologisch-Botanischen Gesellschaft in Wien. Vienna 12 (1/2): 488. TL: Hong Kong, China.

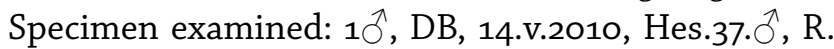
Lodh; 1오 ATNC, 12.iv.2012, Hes.37.ㅇ, R. Lodh.

Conservation status: Tripura: rare; IUCN/CITES: not evaluated.

Distribution: India [Himachal Pradesh in the west to Arunachal Pradesh in the east in Himalaya, Northeast, South India (up to Maharashtra), Tripura, West Bengal), Bhutan, Myanmar, Nepal, Sri Lanka.

210. *Tagiades gana (Moore, 1865); Suffused Snow Flat Pterygospidea gana Moore, 1865, Proceedings of the Zoological Society of London (3): 780. TL: Bengal, India; Java, Indonesia.

Specimen examined: $1 \hat{0}$, SWS, 14.i.2010, Hes.42. $0^{\lambda}$, R. Lodh.

Conservation status: Tripura: rare; IUCN/CITES: not evaluated.

Distribution: India (Northeast, Orissa, Sikkim to Arunachal Pradesh in the east in Himalaya, South India (up to Gujarat), Tripura], Bangladesh, Bhutan, Myanmar, Nepal.

211. Tagiades japetus (Stoll, 1782); Common Snow Flat Papilio japetus Stoll, 1782, Uitlandsche Kapellen (Papillons exotiques) 4 (29-31): 145. TL: Ambon, Indonesia.

Specimen examined: 10 $0^{\lambda}$, TWS, 18.v.2010, Hes.43.0 R. Lodh; 1 ,, DB, 7.iv.2010, Hes.44.우, R. Lodh.

Conservation status: Tripura: common; IUCN/CITES: not evaluated.

Distribution: India (Madhya Pradesh to West Bengal, Northeast, South India north to Gujarat, Tripura, Uttaranchal in the west to Arunachal Pradesh in the east in Himalaya), Bangladesh, Bhutan, Myanmar, Nepal, Sri Lanka.

\section{Tribe Carcharodini}

212. *Spialia galba (Fabricius, 1793); Indian Skipper

Hesperia galba Fabricius, 1793, Entomologia systematica 3(1): 352. TL: Tranquebar, South India.

Specimen examined: 10 , RWS, 18.viii.2009, Hes.41. $0^{\text {, }}$ R. Lodh; 19, RWS, 18.viii.2009, Hes.41. + , R. Lodh.

Conservation status: Tripura: rare; IUCN/CITES: not evaluated.

Distribution: India (common in almost all states of India, Tripura), Bangladesh, Myanmar, Pakistan, Sri Lanka.

\section{DISCUSSION}

The butterfly fauna reported here is found to be rich and an important asset for conservation in this part of Southeast Asia. Altogether, 65 butterfly species (30.66\% of the recorded species) are found to be threatened, including 41 species protected by law in India under
Schedules I (9 species), II (27 species) and IV (5 species) of IWPA 1972 (Anonymous 1997). Another 38 species are categorized as critically endangered (CR), endangered (EN), vulnerable (VU), or near threatened (NT) according to IUCN Red Lists either at the global scale or at regional scale (IUCN 2007; Anonymous 2007). One species, Troides helena (Linnaeus, 1758), is protected under Appendix II G of CITES (UNEP-WCMC 2011). Fifteen species are threatened in India as well as at global scale. Interestingly, six species - T. helena, protected by CITES, Castalius rosimon (Fabricius, 1775) and Papilio memnon Linnaeus, 1758, protected by IWPA 1972 (schedule I), Papilio nephelus Boisduval 1836 and Tanaecia lepidea protected by IWPA 1972 (schedule II), and Udaspes folus (Cramer 1775), protected by IUCN - are found to be abundant in Tripura. These species receive the highest level of protection in other parts of the world. According to species' abundance data recorded in this study, 141 species are found to be rare or very rare in Tripura province including 56 species which are protected by the IWPA (1972) and the IUCN. However, the rest of the 85 species are considered to be of either least concern (LC) or not evaluated for their conservation status according to IUCN. Conservation efforts for these 85 speies are required in view of increasing threats of anthropogenic disturbances that are faced by this biogeographic zone in general and Tripura province in particular. Two sampling sites, Debbari (DB) and Ballianchip ( $\mathrm{BC}$ ), were found to be the most species rich locations each of which supported 112 and 94 species, respectively, and together shared 132 species representing $62.26 \%$ of the recorded butterfly fauna of Tripura. Each of the two locations contained 7 and 6 species, respectively, as unique with regard to their exclusive distribution in these locations in Tripura. Eight of the 65 species recorded in threatened categories from Tripura were collected from these locations.

Thus, Tripura province not only possesses rich butterfly diversity but it also supports a large number of rare and legally protected species and is, therefore, an important destination of study of butterflies and other wildlife in the tropics of Southeast Asia.

\section{ACKNOWLEDGEMENTS}

We thank Sri Dipankar Kishore Sinha and Dr. P.P. Bhattacharjee for their helps during field survey and laboratory work. This study was financed partly by the Japan International Co-operation Agency, Tripura, and partly by the Indian Council of Agricultural Research, New Delhi, for which authors are thankful to Dr. A. K. Gupta, Chief Principal Conservator of Forest (Wildlife), Tripura, and to Dr. V.V. Ramamurthy, National Coordinator of the National Project on Insect Biosystematics. Authors gratefully acknowledge the constructive comments of three reviewers, the subject editor and the 
graphic editor for significant improvements in the manauscript.

\section{LITERATURE CITED}

Ackery, P. R. 1984. Systematic and faunistic studies on butterflies; pp. 9-21, in R. I. Van-Wright and P. R. Ackery (eds.), The biology of butterflies. London; Academic Press; Symposium of the Royal Entomological Society of London (11): 429 pp. http://www. butterfliesofamerica.com/docs/JPP-Bib2008.pdf

Agarwala, B.K., S. Roy Choudhury and P. Ray Chaudhury. 2010. Species richness and diversity of butterflies in urban and natural locations of north-east India. Entomon 35(2): 87-91. http:// entomon.in/index.php/Entomon/about

Anonymous. 1997. The Wildlife (Protection) Act, 1972 (as amended up to 1993) with rules uptil 1995. Dehra Dun: Natraj Publishers. $158 \mathrm{pp}$.

Anonymous. 2007. Red List of Threatened Mammals of Japan; Ministry of the Environment, Government of Japan. Accessed at http://www.nationalredlist.org/species Search/, captured on 30 April 2013.

Anonymous. 2015. The Global Lepidoptera Names Index, Natural History Museum. Accessed at http://www.nhm.ac.uk/research curation/research/projects/lepindex/search/index.dsml, 6 February 2015.

Bhattacharjee, P.P., R. Lodh, J. Majumder, D. Laskar and B.K. Agarwala. 2013. An ornithological survey in the vicinity of Agartala city of Tripura state, northeast India. Journal of Research in Biology 3(3): 852-86o. http://jresearchbiology.com/documents/RAo328.pdf

Campbell, D.L., A.V.Z. Brower and N.E. Pierce. 2000. Molecular evolution of the wingless gene and its implications for the phylogenetic placement of the butterfly family Riodinidae (Lepidoptera: Papilionoidea). Molecular Biology and Evolution 17(5): 684-696. http://mbe.oxfordjournals.org/content/17/5/684. abstract

Choudhury, A. 2010. Recent ornithological records from Tripura, north-eastern India, with an annotated checklist. Indian Birds 6(3): 66-74.

Datta-Roy, A., M. Singh, C. Srinivasha and P.K. Karanth. 2012. Phylogeny of Asian Eutropis (Squamata: Scincidae) reveals an 'into India' endemic Indian Radiation. Molecular Phylogenetics and Evolution 63(3): 817-824. doi: 10.1016/j.ympev.2012.02.022

Deb, D.B. 1981-1983. The Flora of Tripura State: New Delhi: Today and Tomorrows' Printers and Publishers. Vol. I: xviii + 509 pp., Vol. 2: $\mathrm{xi}+601 \mathrm{pp}$.

Ghazoul, J. 2002. Impact of logging on the richness and diversity of forest butterflies in a tropical dry forest in Thailand. Biodiversity Conservation 11(3): 521-541. doi: 10.1023/A:1014812701423

Gogoi, M.J. 2012. Butterflies (Lepidoptera) of Dibang Valley, Mishmi Hills, Arunachal Pradesh, India. Journal of Threatened Taxa 4(12): 3137-316o. doi: 10.11609/JoTT.02975.3137-6o

Gupta, A.K.1998. Status and management of wildlife in Tripura. Indian Forester 124(10): 787-793. http://www.indianforester. co.in/index.php/indianforester/article/view/5763

Gupta, I.J. and D.K. Mondal. 2005. Red Data Book (Part 2): Butterflies of India. Kolkata: Director, Zoological Survey of India. 535 pp.

Gupta, I.J. and D.R. Maulik. 2007. State Fauna Series 14; pp. 413-426, in: Director (ed.) Fauna of Mizoram (Insecta: Lepidoptera). Kolkata, India: Zoological Survey of India.

Gupta, I.J. and M. Majumder. 2006. State Fauna Series 12; pp. 291350, in: Director (ed.) Fauna of Nagaland (Insecta: Lepidoptera). Kolkata, India: Zoological Survey of India.

Haribal, M. 1992. The butterflies of Sikkim Himalaya and their natural history. Sikkim: Sikkim Nature Conservation Foundation. 217 pp.

Heppner, J. 1998. Classification of Lepidoptera. Part I. Introduction. Holarctic Lepidoptera 5(1): 1-148.
IUCN. 2007. The 2007 Red List of Threatened Fauna and Flora of Sri Lanka. Colombo, Sri Lanka: The World Conservation Union (IUCN) and Ministry of Environment and Natural Resources. 148 pp. http://cmsdata.iucn.org/downloads/rl_548_7_oo3.pdf

Kehimkar, I. 2008. The Book of Indian Butterflies. Mumbai: Oxford University Press. 497 pp.

Keshari, G.K. 2011. Geology and Mineral Resources of Manipur, Mizoram, Nagaland and Tripura. Geological survey of India, Government of India. Guwahati: Essar Offset. 104 pp.

Kocher, S.D. and E.H. Williams. 20oo. The diversity and abundance of North American butterflies vary with habitat disturbance and geography. Journal of Biogeography 27(4): 785-794. doi: 10.1046/j.1365-2699.2000.00454.x

Kunte, K. 2000. Butterflies of Peninsular India. Hyderabad: Universities Press. 272 pp.

Kunte, K., S. Sondhi, B.M. Sangma, R. Lovalekar, K. Tokekar and G. Agavekar. 2012. Butterflies of the Garo Hills of Meghalaya, northeastern India: their diversity and conservation. Journal of Threatened Taxa 4(10): 2933-2992. http://www.threatenedtaxa. in/index.php/JoTT/article/view/799/1433

Kunte, K., S. Kalesh and U. Kodandaramaiah. 2014. Butterflies of India, v. 2.10. Indian Foundation for Butterflies. Accessed at http://www.ifoundbutterflies.org, 13 April 2013.

Larsen, T.H., F. Escobar and I. Armbrecht. 2011. Insects of the tropical Andes: Diversity patterns, processes and global change; pp. 228-244, in S.K. Herzog, R. Martínez, P.M. Jorgensen and $\mathrm{H}$. Tiessen (eds.). Climate Change and Biodiversity in the Tropical Andes. Brazil: Inter-American Institute for Global Change Research (IAI) and Scientific Committee on Problems of the Environment (SCOPE). http://www.iai.int/files/ communications/publications/scientific/Climate_Change_and_ Biodiversity_in_the_Tropical_Andes/book.pdf

Majumdar, K., B.K. Datta and U.Shankar. 2012b. Ten new additions of tree species to the flora of Tripura state, North East India: Distributional range extension and geographical map. NeBio 3(1): 17-24. http://nebio.in/cgi-sys/suspendedpage.cgi

Majumdar, K., U. Shankar and B. K. Datta. 2012a. Tree species diversity and stand structure along major community types in lowland primary and secondary moist deciduous forests in Tripura, Northeast India. Journal of Forestry Research 23(4): 563-568. doi: 10.1007/s11676-012-0295-8

Majumder, J., P.P.Bhattacharjee, K. Majumdar, C. Debnath and B.K.Agarwala.2012a. Documentation of herpatofaunal species richness in Tripura, Northeast India. NeBio 3(1): 60-70.

Majumder, J., R. Lodh, B.K. Agarwala. 2012b. Variation in butterfly diversity and unique species richness along different habitats in Trishna Wildlife Sanctuary, Tripura, northeast India. Check List 8(3): 432-436. http://www.checklist.org.br/getpdf?SL108-11

Martinez, A.L., J.L. Bousquets, I.F. Fernandez and A.D. Warren. 2003. Biodiversity and Biogeography of Mexican butterflies (Lepidoptera: Papilionoidea and Hesperioidea). Proceedings of Entomological Society of Washington 105(1): 209-244. http:// www.biodiversitylibrary.org/item/54811

May, P.G. 1992. Flower selection and the dynamics of lipid reserves in two nectarivorous butterflies. Ecology 73(6): 2181-2191. doi: $10.2307 / 1941466$

Mittermeier, R.A., P.R. Gill, M. Hoffman, J. Pilgrim, T. Brooks, C.G. Mittermeier, J. Lamoreux and G.A.B. da Fonseca. 2005. Hotspots Revisited: Earth's Biologically Richest and most Endangered Terrestrial Ecoregions. Mexico: CEMEX. 392 pp.

Myers, N., A. Russell, C. Mittermelert, G. Mittermelert, A.B. Gustavo and K.J. Fonseca. 2000. Biodiversity hotspots for conservation priorities. Nature 403: 853-858. doi: 10.1038/35002501

Naeem, S., L.J. Thompson, S.P. Lawler, J.H. Lawron and R.M.Woodfin. 1994. Declining biodiversity can alter the performance of ecosystem. Nature 368: 734-736. doi: 10.1038/368734ao 
Pollard, E. 1977. A method for assessing changes in the abundance of butterflies. Biological Conservation 12(2): 115-134. http://www. sciencedirect.com/science/journal/ooo63207/12/2

Roy Choudhury, S., P. Ray Choudhury and B.K. Agarwala. 2011. Butterflies of Trishna Wildlife Sanctuary of North-East India with a note on their Diversity and Seasonality; pp. 261-265. in (compls.) Proceedings of National Conference on Water, Energy and Biodiversity with Special Reference to North-East Region. Agartala, India: The Engineers Association of India, Tripura Chapter.

Samways, M.J. 2005. Insect diversity conservation. Cambridge: Cambridge University Press. 356 pp.

Savela, M. 2013. Lepidoptera and some other life forms. Accessed at http://www.nic.funet.fi/pub/sci/bio/life/lepidoptera/ditrysia/ index.html, 13 April 2013.

Spector, S. 2006. Scarabaeine dung beetles (Coleoptera: Scarabaeidae: Scarabaeinae): an invertebrate focal taxon for biodiversity research and conservation. Coleopterists Bulletin 6o(5): 71-83. doi: 10.1649/0010-065X(2006)6o[71:SDBCSS]2.0.CO;2

Tilman, D., D. Wedin and J. Knops. 1996. Productivity and sustainability influenced by biodiversity in grassland ecosystem. Nature
379: 718-720. doi: 10.1038/379718ao

Tordoff, A.W., M.C. Baltzer, J.R. Fellowes, J.D. Pilgrim and P.F. Langhammer. 2012. Key Biodiversity Areas in the IndoMyanmar Hotspot: Process, Progress and Future Directions. Journal of Threatened Taxa 4(8): 2779-2787. doi: 10.116o9/JoTT. 03000.2779-87

UNEP-WCMC. 2011. Checklist of CITES species [CD-ROM]. CITES Secretariat, Geneva, Switzerland, and UNEP-WCMC. Cambridge: United Kingdom.

Winston, J.E. 1999. Describing species: practical taxonomic procedure for biologist. Columbia: Columbia University Press. 512 pp.

Authors' contribution statement: RL collected the specimens and did photography, RL and BKA did identification, wrote the text, and attended the review process.

Received: April 2014

Accepted: January 2015

Editorial responsibility: Cristiano $\mathrm{A}$. Iserhard 